\title{
Aeroelastic Analysis Of Membrane Wings
}

by

\author{
Soumitra Pinak Banerjee
}

A Thesis submitted to the Faculty of Virginia Polytechnic Institute and State University in partial fulfillment of the requirements for the degree of

\section{MASTER OF SCIENCE}

in

Aerospace Engineering

APPROVED

\author{
Mayuresh J. Patil, Chair \\ William J. Devenport \\ Rakesh K. Kapania
}

August 22, 2007

Blacksburg, Virginia

Keywords: Vortex lattice method, membranes, MAV, Aeroelasticity, Flapping Wings copyright (C)Soumitra Pinak Banerjee 


\title{
Aeroelastic analysis of membrane wings
}

\author{
Soumitra Pinak Banerjee
}

\section{$\underline{\text { Abstract }}$}

The physics of flapping is very important in the design of MAVs. As MAVs cannot have an engine that produces the amount of thrust required for forward flight, and yet be light weight, harnessing thrust and lift from flapping is imperative for its design and development. In this thesis, aerodynamics of pitch and plunge are simulated using a 3-D, free wake, vortex lattice method (VLM), and structural characteristics of the wing are simulated as a membrane supported by a rigid frame. The aerodynamics is validated by comparing the results from the VLM model for constant angle of attack flight, pitching flight and plunging flight with analytical results, existing 2-D VLM and a doublet lattice method. The aeroelasticity is studied by varying parameters affecting the flow as well as parameters affecting the structure. The parametric studies are performed for cases of constant angle of attack, plunge and, pitch and plunge. The response of the aeroelastic model to the changes in the parameters are analyzed and documented. The results show that the aerodynamic loads increase for increased deformation, and vice-versa. For a wing with rigid boundaries supporting a membranous structure with a step change in angle of attack, the membrane oscillates about the steady state deformation and influence the 
loads. For prescribed oscillations in pitch and plunge, the membrane deformations and loads transition into a periodic steady state. 


\section{Acknowledgments}

I would like to express my sincere appreciation to Dr. Mayuresh J. Patil, for the opportunity to work for him, the support, continual help, patience, dedication, and advise. Dr. Patil has been an exceptional mentor for which I will always be grateful to him. I'm very grateful for the knowledge that I've acquired working for him, and being extremely tolerant in explaining and teaching me all I know. I'd also like to thank Dr. Rakesh K. Kapania, and Dr. William J. Devenport for serving on my committee.

I would like to thank my parents, Pinak and Bharati Banerjee, my brother Indranil Banerjee for their incessant support and encouragement. They've always been my driving force.

Last but not the least, I'd like to thank my friends and colleagues here at Virginia Tech, for making a sociable working environment. I'd specially like to thank Brijesh Raghavan. I've learned a lot from him. 


\section{Contents}

1 Introduction and Overview 1

1.1 Motivation . . . . . . . . . . . . . . . . . 1

1.2 Micro Air Vehicles (MAVs) . . . . . . . . . . . . . . 2

1.3 Aerodynamic modeling . . . . . . . . . . . . . . . . . . 4

1.4 Aero-Structures interation $\ldots \ldots \ldots \ldots \ldots$

1.5 Thesis Layout $\ldots \ldots \ldots \ldots$

2 Aerodynamic Modeling $\quad 11$

2.1 Basic Concepts . . . . . . . . . . . . . . . . . . 13

2.1.1 Angular Velocity, Vorticity, and Circulation . . . . . . . 13

2.1 .2 Irrotationality . . . . . . . . . . . . . . . 15

2.2 Biot-Savart Law . . . . . . . . . . . . . . . . . . . . . 16

2.2.1 Velocity Induced by a Straight Vortex Section . . . . . . . 20 
2.3 Vortex Lattice Method . . . . . . . . . . . . . . . . . . . . 22

2.3.1 Spatial Conservation of Vorticity _. . . . . . . . . . 22

2.3.2 Helmholtz's theorem . . . . . . . . . . . . . . . . . . . 24

2.3 .3 Kelvin's theorem . . . . . . . . . . . . . . . . . 25

2.3.4 Discretized Vortex Sheet . . . . . . . . . . . . . . 26

2.3 .5 Boundary conditions $\ldots \ldots \ldots \ldots$

2.3.6 Calculation of Load Coefficient . . . . . . . . . . 34

2.3 .7 Implementation of VLM $\ldots \ldots \ldots \ldots$

2.4 Theoretical Results . . . . . . . . . . . . . . . . . 45

3 Structural Modeling And Aeroelasticity 48

3.1 Membrane theory . . . . . . . . . . . . . . . . . . . . 49

3.1.1 Static Membrane Deformation . . . . . . . . . . . . 51

3.2 Application of Fourier Series . . . . . . . . . . . . . . . 51

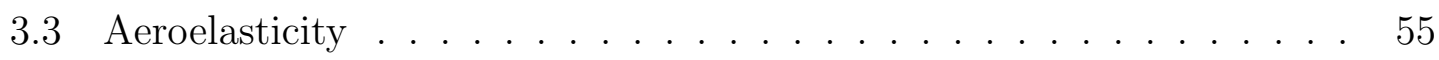

3.3 .1 Time $t=t_{1} \ldots \ldots \ldots \ldots \ldots \ldots \ldots \ldots \ldots$

3.3 .2 Time $t_{i}>t_{1} \ldots \ldots \ldots \ldots \ldots \ldots$

4 Results $\quad 59$

4.1 Validation of the 2-dimensional aspects of the Aerodynamic model . . 59 
4.1.1 Constant Angle of Attack _. . . . . . . . . . . . . 60

4.1 .2 Pitching wing . . . . . . . . . . . . . . 65

4.1 .3 Plunging wing $\ldots \ldots \ldots \ldots 6 \ldots$

4.2 Grid discretization studies . . . . . . . . . . . . . . 68

4.3 Comparison with lifting line theory $\ldots \ldots \ldots \ldots$

4.4 Validation of 3-dimensional aspects of VLM with Doublet Lattice

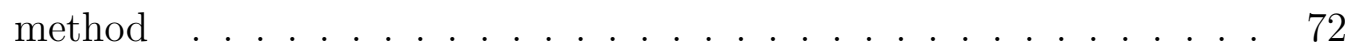

4.4 .1 Pitch . . . . . . . . . . . . . . . . . . . 72

4.4 .2 Plunge . . . . . . . . . . . . . . . . . . . 75

4.5 Study of loads and deformations for constant angle of attack . . . . 75

4.5.1 Variation in the number of structural modes . . . . . . . 76

4.5 .2 Variation in stiffness $\ldots \ldots \ldots \ldots$

4.6 Study of loads and deformations for a plunging wing . . . . . . . 84

4.6.1 Variation in the number of structural modes . . . . . . . 84

4.6.2 Variation in plunge amplitude . . . . . . . . . . . . . 89

4.6.3 Variation in reduced frequency . . . . . . . . . . . . 91

4.6.4 Variation in stiffness . . . . . . . . . . . . . . 93

4.7 Study of loads for a pitching and plunging wing at different phases . . 96

$\begin{array}{lll}5 \text { Conclusion } & 100\end{array}$ 
6 Bibliography

103

viii 


\section{List of Figures}

2.1 Relation between Surface To Line Integrals . . . . . . . . . . . . . . . 14

2.2 Velocity at point $L$ due to a vortex distribution . . . . . . . . . . 18

2.3 Velocity at point $L$ induced by a vortex segment . . . . . . . . . . . . 19

2.4 Velocity Induced By A Straight Vortex Segment . . . . . . . . . . . . 20

2.5 Nomenclature used for the velocity induced by a three-dimensional, straight vortex segment ................. . . 21

2.6 Chordwise And Spanwise Running Vortex Segments . . . . . . . . . 22

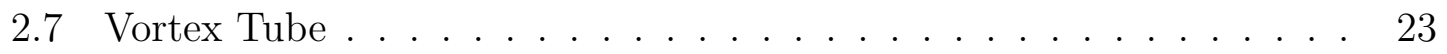

2.8 Sum of $\Gamma$ at a node is zero . . . . . . . . . . . . . . 26

2.9 Rectangular Wing with Vortex Rings . . . . . . . . . . . . . . 27

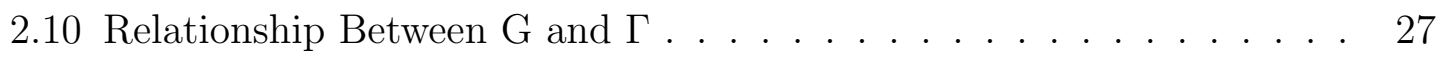

2.11 One Row of Shed Vortex Rings . . . . . . . . . . . . . . . . 29

2.12 Calculation of Normal Vector . . . . . . . . . . . . . . . . 32 
2.13 Anatomy of a vortex element $\ldots \ldots \ldots \ldots \ldots$

2.14 Description of Grid at times $t$ and $t+\Delta t \ldots \ldots \ldots \ldots$

3.1 Description of Membrane And Membrane Deflection . . . . . . . . . 49

3.2 Rectangular Wing . . . . . . . . . . . . . . . . . . . . 52

4.12 -D plot of the wake vortices after 50 time steps $\ldots \ldots \ldots$

4.2 2-D plot of the wake vortices after 50 time steps with equal axis . . 61

4.3 Comparison of Vortex strengths of 2-D and 3-D VLM after 50 time steps ............................ 61

4.4 Comparison of 2-D Lift coefficient as a function of time $\ldots \ldots \ldots$

4.5 Comparison of Vortex Strengths of 2-D and 3-D VLM . . . . . . . 64

4.6 2-D plot of the location of the wake vortices after 150 time steps . . . 64

4.7 2-D lift coefficient developed by 2-D and 3-D VLM as a function of time 65

4.8 Comparison of Circulation of 2-D and 3-D VLM . . . . . . . 66

4.92 -D plot of the location of the wake vortices after 150 time steps . . 67

4.10 2-D lift coefficient developed by 2-D and 3-D VLM . . . . . 67

$4.11 C_{l}$ from grid discretization study for wing in oscillating plunge $\ldots .68$

$4.12 \Gamma$ from grid discretization study for wing in oscillating plunge $\ldots . .69$

$4.13 C_{l}$ from grid discretization study for wing in oscillating pitch . . . . 69

$4.14 \Gamma$ from grid discretization study for wing in oscillating pitch $\ldots \ldots 70$ 
4.15 Plot of the spanwise circulation for $\alpha=5^{0} \ldots \ldots \ldots 71$

4.16 Plot of $2-\mathrm{D} C_{l}$ for $\alpha=5^{0} \ldots \ldots \ldots \ldots \ldots \ldots \ldots \ldots \ldots$

4.17 Comparison of $C_{l}$ from 3-D VLM and DLM for pitch $\ldots \ldots . .73$

4.18 Comparison of the phase angle between the input pitch and the $C_{l} \ldots 73$

4.19 Comparison of $C_{l}$ from 3-D VLM and DLM for plunge $\ldots \ldots \ldots .74$

4.20 Comparison of the phase angle between the plunge and the $\mathrm{Cl} \ldots 74$

4.21 Plot of $C_{l}$ versus time for constant angle of attack of $5^{\circ}$ and $\mathrm{S}=10 \ldots 76$

4.22 Plot of $C_{d}$ versus time for angle of attack of $5^{\circ}$ and $\mathrm{S}=10 \ldots \ldots 77$

4.23 Plot of $w$ versus time at approximately the center of the wing for angle of attack of $5^{\circ}$ and $\mathrm{S}=10 \ldots \ldots \ldots \ldots \ldots$

4.24 Plot of the deformation at the midspan of the wing for an angle of attack of $5^{\circ}$ and $\mathrm{S}=10 \ldots \ldots \ldots \ldots \ldots \ldots$

4.25 Plot of $C_{l}$ versus time for constant angle of attack of $5^{\circ} \ldots \ldots$. . 81

4.26 Plot of $C_{d}$ versus time for angle of attack of $5^{\circ} \ldots \ldots \ldots 1$

4.27 Plot of $w$ versus time at approximately the center of the wing for angle

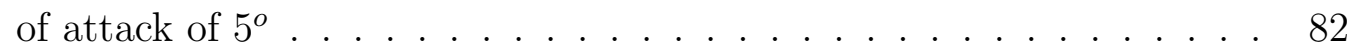

4.28 Plot of the deformation at the midspan of the wing for an angle of attack of $5^{\circ} \ldots \ldots \ldots \ldots \ldots \ldots \ldots \ldots \ldots \ldots$ 
4.29 Plot of $C_{l}$ Vs Time for different number of structural modes for a plunging wing . . . . . . . . . . . . . . . . . 85

4.30 Plot of $C_{d}$ Vs Time for different number of structural modes for a

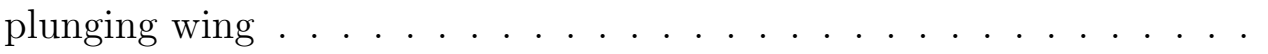

4.31 Plot of wing's center Vs Time for different number of structural modes for a plunging wing . . . . . . . . . . . . . . . . 86

4.32 Plot of $C_{l}$ Vs Time for different plunge amplitudes for a plunging wing 88

4.33 Plot of $C_{d}$ Vs Time for different plunge amplitudes for a plunging wing 88

4.34 Plot of Wing's center Vs Time for different plunge amplitudes for a plunging wing ........................... 89

4.35 Plot of $C_{l}$ Vs Time for different reduced frequencies for a plunging wing 91

4.36 Plot of $C_{d}$ Vs Time for different reduced frequencies for a plunging wing 92

4.37 Plot of Wing's center Vs Time for different reduced frequencies for a plunging wing ...................... . . . 92

4.38 Plot of $C_{l}$ Vs Time for different pre-stresses for a plunging wing . . . 94

4.39 Plot of $C_{d}$ Vs Time for different pre-stresses for a plunging wing . . . 94

4.40 Plot of Wing's center Vs Time for different pre-stresses for a plunging

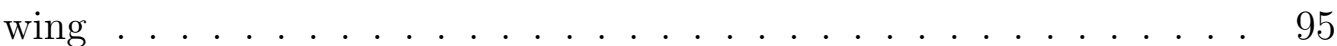

4.41 Comparison of $C_{l}$ for a pitching and plunging wing at different phases 96 
4.42 Comparison of $C_{d}$ for a pitching and plunging wing at different phases 97

4.43 Comparison of $w$ for a pitching and plunging wing at different phases $\quad 97$ 


\section{Chapter 1}

\section{Introduction and Overview}

\subsection{Motivation}

Birds and insects use flapping wing mechanics to fly, by simultaneously producing thrust and lift. The flight of birds and insects are different. During a flapping cycle, most birds flap their wings in a vertical plane with small changes in the pitch of their wings. To produce lift birds use forward velocity. Thus, most birds cannot hover. Insects, on the other hand, flap their wings in a nearly horizontal plane, with high pitch angles. This enables them to produce lift in the absence of forward velocity [1]. Micro Air Vehicles (MAVs) are being designed keeping flapping wing mechanics

in hindsight. MAVs have been successfully built and tested at the University of 
Florida MAV Laboratory [2] [3] [4], and the University of Maryland's Alfred Gessow Rotorcraft Center [1]. The goal of the work done in this thesis is to simulate the flight of a rectangular wing that can be used to model the flight of MAVs, and lay down the foundation for other types of MAVs. The preliminary work of simulation would serve as a reference in understanding the physics, and the math that goes behind the development of MAVs. The aerodynamics is simulated using the vortex lattice method, and the structural parameters are approximated using Fourier series.

\subsection{Micro Air Vehicles (MAVs)}

Micro air vehicles are remotely or autonomously controlled unmanned air vehicles (UAV) typically of six inches $(15 \mathrm{~cm})$ in size. MAVs are defined as low aspect ratio wings operating at low Reynold's number of the order of $10^{4}[5]$. In terms of size and

weight, MAVs are defined as being $15 \mathrm{~cm}$ and weighing 15 grams [1]. The idea of micro air vehicles evolved from insects and birds, who use flapping flight mechanics to exploit unsteady aerodynamic effects [5]. However, the current MAVs that use these mechanics have evolved from fixed wing designs, which are easier to design.

A much advanced work has been done by the University of Florida MAV Laboratory. The group has worked on MAVs whose wing span ranged from $8 \mathrm{~cm}$ to $2 \mathrm{~m}$. Their accomplishments include a 4.5 inch MAV that captured images $600 \mathrm{~m}$ away; 
8,12 and 16 inch prototypes of MAVs designed to carry relatively heavier payloads; the Tadpole, which was designed for Low Altitude Geo-Reference Photography; a 6 inch wingspan morphing MAV that could fit into a "Cigar" tube and quickly deploy, and assemble its form; the Pocket Micro Air Vehicle (PMAV), that could fit in a canister, which could fit into military battle dress pant size pockets, and is used for immediate field access for short range reconnaissance; the 12 inch Gator A which was again used for visual search for multiple targets; a 24 inch Bomb Damage assessment (BDA) MAV whose mission was surveillance; and the Micro Morphing Air-Land vehicle, which in addition to its aerial mission of surveillance is also capable of terrestrial locomotions using WHEGs (a method of locomotion that combines the advantages of wheels and legs) to detect IEDs (Improvised explosive devices).

MAVs are designed to be morphing or with fixed wings. It has been shown that fixed wing MAVs have higher endurance than rotary or flapping wing MAVs. The Alfred Gessow Rotorcraft Center at the University of Maryland has used an insect flight mechanism to design MAVs. The MAV Mentor uses the the "clap wing" phenomenon, which is used by a few species of insects. Ornithoptic flapping, which is bird like flapping, has been studied experimentally to a great extent. However, the more convoluted hover-capable flapping could not be tested due to complex design requirements; most literature comprises of research by biologists on the wing 
kinematics and morphology of actual insects [1].

\section{$1.3 \quad$ Aerodynamic modeling}

Various approaches have been pursued by different researchers in modeling the aerodynamics of a MAV or in the study of birds and insects. As in the aerodynamic modeling of birds and insects, flapping is an important aspect of their flight, its imperative to understand and accurately model flapping flight. In birds and insects, vortices are released from the trailing edges of the lifting surface, wings in their case, that contain information of the time history and magnitude of the aerodynamic force. The wake vortices thus act as an aerodynamic footprint of the passage of the flying object[6]. In an experimental study of bat flight [6], it was found that in slow speeds a vortex loop was shed by the wing, whereas in high speeds a pair of undulating vortices were shed that trailed from the wing tip. This qualitative observation let the researchers to conclude that lift was produced both during downstroke as well as upstroke. This was however contradicted by kinematic studies that suggested that a wing tip reversal reversed the wing circulation during upstroke. Some of the important conclusions of this study are; the circulation reaches a zero value while transitioning from upstroke to downstroke, which contrasts the constant circulation wake model used to simulate bird flight; the quantitative data also show that neg- 
ative lift is achieved during upstroke at medium and high speeds. The negative lift could be due to the membranous nature of a bat's wing that prevents separation [6]. The analytical studies done on the aerodynamics of hover-capable flapping wings have mainly been done on rigid wings [7] [8]. Some of these studies have looked at ornithoptic flapping whereas some have looked at insect flight. For the modeling of insect flight, the approaches available for aerodynamic modeling range from indicial methods based on Wagner and Kussner's functions to the computationally intensive unsteady vortex lattice method (UVLM) and CFD analysis [1].

The aerodynamics can also be modeled using particle wake aerodynamics, as explained by Nitzsche and Opoku [9]. Their aerodynamic code, GENUVP, has the potential to simulate various flight situations and rotorcraft configurations and is also capable of accurate modeling of wake effects. The unsteady vortex particle code uses the Helmholtz decomposition principle, through which the flow-field is decomposed into an irrotational part and a rotational part. The rotational part is due to wakes emitted by lifting bodies. The aerodynamic simulation starts out with the calculation of the velocity of the near wake elements which are calculated using the vortex panel method, via the Biot-Savart law. At the next time step, vortex particles are formed from near wake strip elements, thereby becoming part of the 'far-wake'. The strengths of these vortex particles are computed by the integration 
of the vorticity of near-wake elements. Vortex particle methods have been widely used in rotorcraft aerodynamic simulation, and their theory can be found in other publications [10].

Aerodynamics model simulating wake structures generated by rotating blades using vortex blobs have also been pursued [11]. Their experimentally validated results focus on simulated wake geometries in the radial and axial directions. In their simulation of the wake, the wake is divided into three different sections; the tip vortex region which is well defined, an intermediate region, and an initially generated wake bundle. The model developed is a function of the geometry of the rotor blades, and geometries have to be modified for accurate simulations. The vortices shed into the wake are simulated as vortex blobs; which are finite vortex sticks, with a location and an associated strength. The model also takes into account vortex stretching, which is the updated vorticity vector by the local strain field.

The application of vortex-blob method to airfoils with thickness at high Reynold's number can be found in Ref. [12]. According to the author, the vortex-blob method provides a natural and numerically efficient description of eddies and their strengths. Also, the implementation of the method in a Lagrangian frame makes the simulation grid free, and allows modeling around multi element bodies of arbitrary shape. The boundary conditions used in this paper is the same as the ones used for the research 
in this thesis.

CFD codes have been used in the aerodynamic simulation of flow over a wing as well as rotating blades [13]. The generation and transport of the vortices can be described using CFD. However, a rapid decay of the vortical structures is caused by numerical dissipation. The shortcomings and advantages are taken into account before the development of the aerodynamic code by several groups [11]. Describing the far wake boundary condition causes problems in hovering flight and free wake analysis .

To date successful implementation of prescribed wake [14], an interactive freewake and a time marching free-wake model have been done by many researchers [13] [15] [16].

\subsection{Aero-Structures interation}

Of all the studies performed on birds and insects, only the wings of bats and some insects can closely resemble a membranous surface. They can be actively stretched and collapsed, and lack feathers. [6].

As in any aeroelastic problem, the structure and the aerodynamics of a flying body have interactions with each other changing their physical characteristics. This could give rise to aeroelastic instabilities in flight which could have a disastrous 
conclusion. Aeroelastic instabilities can be static or dynamic in nature. The static aeroelastic problems most heard off in the aviation industry are divergence, and control surface reversal. Divergence occurs when the aerodynamic loads deflect the lift surface such that the applied loads increase. The increased loads cause the wings to further deflect and reach the limit loads and could lead to failure. Control surface reversal is the reversal or loss of a control surface, due to the elastic deformations of the lifting surface structure. The dynamic cases most heard off include flutter, buffeting, and dynamic response. Flutter is defined as a dynamic instability occur

Patil [17], explains the difference in flutter and flapping flight using a holistic energy perspective. The paper shows that energy is transferred from propulsion to the structure via the flow during flutter. And thus, as the system goes into flutter there is an increase in the amount of drag. Flutter and flapping flight are both classified as modes of an energy flow. Flutter is an unstable mode resulting in an increase in drag, while flapping flight is a stable and damped mode with energy transferred to be used for propulsion [17].

Aeroelastic analysis of hover-capable, bio-mimetic flapping wings is presented in reference [1]. Biomimetic pitching-flapping mechanisms are studied for flight in air at high frequency. Due to this the light weight and associated high flexibility, the structures undergo significant aeroelastic effects. The structural model takes into account 
the large motion of the wings and is discretized as plate finite elements. The wing consists of mylar sheet with an aluminum external frame. The load and deflection are also calculated using Fourier series and matched reasonably with experimental results. Dynamic aeroelastic analysis of morphing wings has been done with the aid of neural networks [18].

\subsection{Thesis Layout}

Chapter 1 of the thesis goes through the various research work done in the field of numerical modeling of unsteady flows, theoretical as well as experimental work done in the field of MAVs and morphing air vehicles, and works in the field of aeroelasticity. Chapter 2 elaborates on the approach taken in this thesis in aerodynamic modeling. It goes over the theory that leads to the development of the vortex lattice method and describes the details of the VLM. Chapter 3 focuses on the structural theory and the coupling of the aerodynamic and structural models. It emphasizes the aeroelasticity and describes the order of accuracy of the differencing schemes employed. Chapter 4 consists of the results generated from the work, including validation studies for the aerodynamic model. Chapter 5 is the conclusion, and summarizes all that could be deduced from the research. ling of unsteady flows, theoretical as well as experimental work done in the field of MAVs and morphing air vehicles, and works in the 
field of aeroelasticity. Chapter 2 elaborates on the approach taken in this thesis in aerodynamic modeling. It goes over the theory that leads to the development of the vortex lattice method and describes the details of the VLM. Chapter 3 focuses on the structural theory and the coupling of the aerodynamic and structural models. It emphasizes the aeroelasticity and describes the order of accuracy of the differencing schemes employed. Chapter 4 consists of the results generated from the work, including validation studies for the aerodynamic model. Chapter 5 is the conclusion, and summarizes all that could be deduced from the research. 


\section{Chapter 2}

\section{Aerodynamic Modeling}

The aerodynamic model is designed to encompass low-speed, low-angle of attack, but large motions. The model is developed for inviscid, incompressible, and unsteady flow. The low-angle of attack, and low-speed assumption ensures that the flow separates at the trailing edge. To design a model, the vortex lattice method with vortex rings is implemented. An ideal model would take viscous effects into consideration, and would be based on the Navier-Stokes equations. Other ways of modeling the flow would be based on either vortex particle method, free vortex blob method, and computational fluid dynamics.

CFD programs can simulate the case being studied with higher fidelity, as compared to vortex lattice methods or, vortex particle methods. More sophisticated 
codes available in the industry based on vortex lattice methods can also do a very good job of simulating the flow. However, the purpose of this study is to do a preliminary aerodynamic analysis. Since the flow parameters considered are inviscid; with small angle of attack, and pitch angle; and low speed; the vortex lattice method is a reliable method for preliminary analysis. Vortex lattice methods can be implemented with a fixed wake or a free wake approximation. Since the method is being developed for cases of pitch and plunge; and has to be general enough to accommodate flapping wing effects for future work, the free-wake model's wake shape works as a check point to validate whether the physics of the flight is being captured by the model. Also, the vortex stretching terms included in the free-vortex blob methods are not considered as the vortex stretching effects are insignificant for the cases being studied. The VLM is inexpensive medium order, medium fidelity model. The study and application of CFD methods, and sophisticated simulation softwares require experience, and are expensive.

The Vortex Lattice Method is described below. 


\subsection{Basic Concepts}

\subsubsection{Angular Velocity, Vorticity, and Circulation}

The angular motion of a fluid element consists of translation, rotation, and deformation. The translation is caused by the local velocity of the fluid. Due to variations in the local velocity, the fluid element rotates and deforms. The components of the angular velocity of the fluid are given by [14],

$$
\omega_{k}=\frac{1}{2}\left(\frac{\partial V_{i}}{\partial x_{j}}-\frac{\partial V_{j}}{\partial x_{i}}\right) \epsilon_{i j k}
$$

where, indices $i, j$ and $k$ represent the three directions of the cartesian frame, and $i \neq j \neq k$.

The angular velocity can thus be written as,

$$
\vec{\omega}=\frac{1}{2}(\vec{\nabla} \times \vec{V})
$$


For convenience, the term vorticity is defined as,

$$
\begin{aligned}
\vec{\zeta} & =2 \vec{\omega}=\vec{\nabla} \times \vec{V} \\
\zeta_{k} & =2 \omega_{k}=\left(\frac{\partial V_{i}}{\partial x_{j}}-\frac{\partial V_{j}}{\partial x_{i}}\right)
\end{aligned}
$$

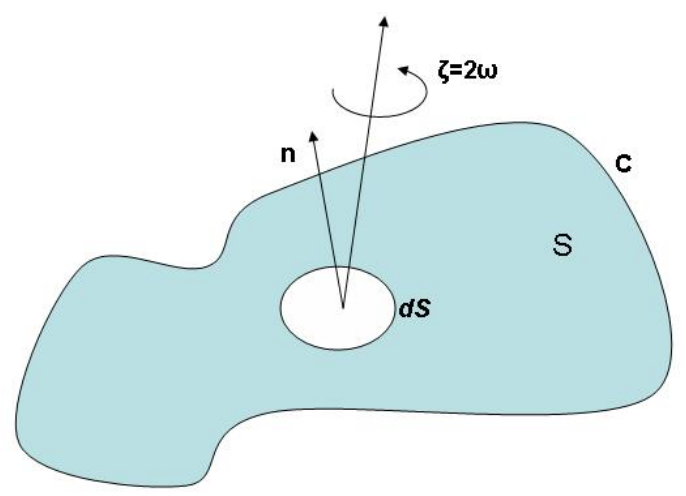

Figure 2.1: Relation between Surface To Line Integrals

For an open surface $S$ with a closed curve $C$ along its boundary shown in Figure 2.1, the vorticity on the surface $S$ can be related to the line integral around curve $C$ using Stokes's theorem.

$$
\int_{S} \vec{\nabla} \times \vec{V} \cdot \vec{n} d S=\int_{S} \vec{\zeta} \cdot \vec{n} d S=\oint_{C} \vec{V} \cdot d l
$$

where $\vec{n}$ is normal to the surface $d S$. This term is called the Circulation and is 
represented by the Greek letter $\Gamma$.

$$
\Gamma=\oint_{C} \vec{V} \cdot d l
$$

The Circulation establishes the relationship with the velocity, and is later used in the computation of aerodynamic loads.

\subsubsection{Irrotationality}

In the presence of viscous forces, a fluid particle will rotate like a rigid body. This flow is called rotational, and

$$
\vec{\nabla} \times \vec{V} \neq 0
$$

On the other hand, in the absence of large viscous forces as being considered in the aerodynamic modeling, which exist in the region outside the boundary layer of a body in motion, the fluid is irrotational. 


$$
\vec{\nabla} \times \vec{V}=0
$$

\subsection{Biot-Savart Law}

For an incompressible fluid, the continuity equation is in the form of Laplace's equation. [14].

$$
\vec{\nabla} \cdot \vec{V}=0
$$

The following steps are taken to establish a relationship in between the velocity and a known vorticity distribution. In a region where vorticity can exist, the velocity field is expressed as the curl of a vector field $\mathbf{B}$ such that,

$$
\vec{V}=\vec{\nabla} \times \vec{B}
$$

$\mathbf{B}$ is indeterminate to within the gradient of a scalar function of position and 
time, as the curl of a gradient vector is zero. $\mathbf{B}$ can further be selected to satisfy,

$$
\vec{\nabla} \cdot \vec{B}=0
$$

Eq. 2.3 in conjunction with Eq. 2.10 can be written as,

$$
\vec{\zeta}=\vec{\nabla} \times \vec{\nabla} \times \vec{B}=\vec{\nabla}(\vec{\nabla} \cdot \vec{B})-\nabla^{2} \vec{B}
$$

By applying 2.11 into the above equation, it is further reduced to

$$
\vec{\zeta}=-\nabla^{2} \vec{B}
$$

Using Green's theorem the solution to this equation is (Ref. [19], pp.533),

$$
\vec{B}=\frac{1}{4 \pi} \int_{Q} \frac{\vec{\zeta}}{\left|r_{0}-r_{1}\right|} d Q
$$

where $Q$ is the volume. 


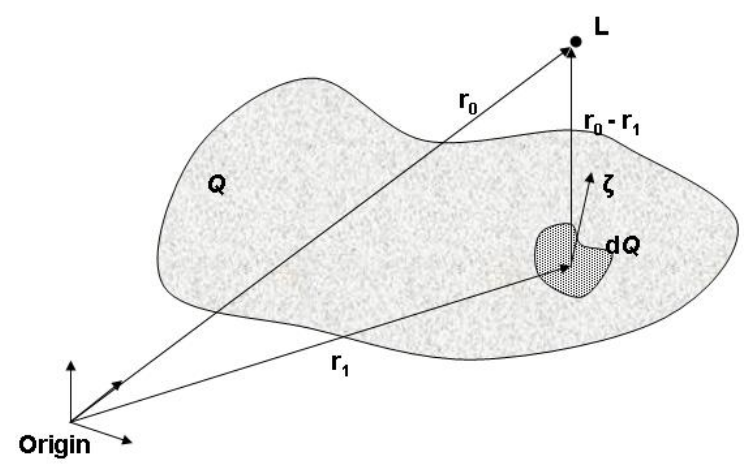

Figure 2.2: Velocity at point $L$ due to a vortex distribution

As shown in Figure 2.2, at a point $L$, the velocity is then a curl of vector $\mathbf{B}$ :

$$
\vec{V}=\frac{1}{4 \pi} \int_{Q} \vec{\nabla} \times \frac{\vec{\zeta}}{\left|\overrightarrow{r_{0}}-\overrightarrow{r_{1}}\right|} d Q
$$

where $r_{0}$ is the distance from the origin, with the vorticity at a distance of $r_{1}$ and $Q$ is the volume containing the vorticity

The velocity due to a volume distribution of vorticity is derived below. Consider an infinitesimal vorticity element $\zeta$, Figure 2.3 . The cross sectional area $d S$ is selected such that the vorticity vector $\zeta$ is perpendicular to it. The direction of the filament $d \mathbf{l}$ is parallel to the vorticity vector and is expressed as, 


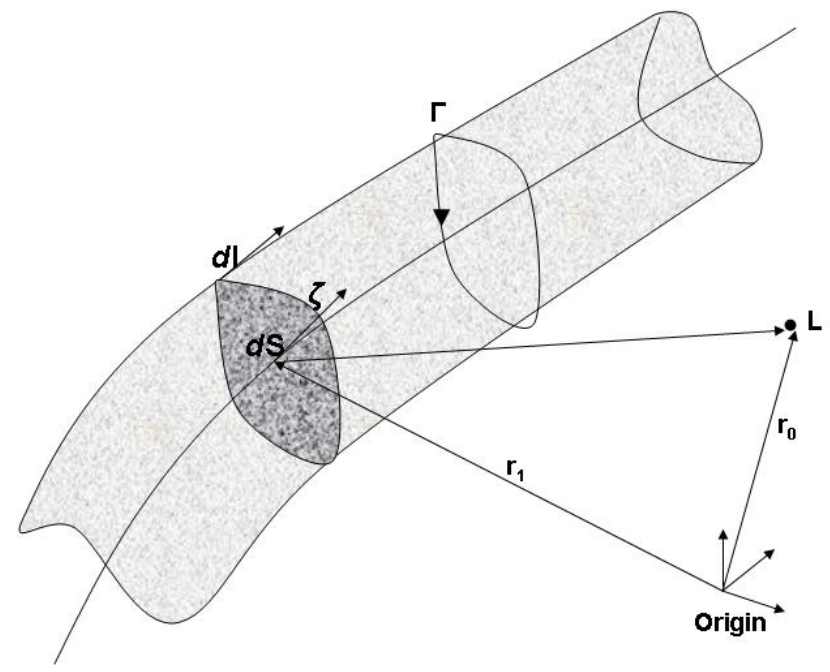

Figure 2.3: Velocity at point $L$ induced by a vortex segment

$$
\overrightarrow{d \mathbf{l}}=\frac{\vec{\zeta}}{\zeta} d l
$$

The circulation is thus given by,

$$
\begin{aligned}
\vec{\Gamma} & =\vec{\zeta} d S \\
d Q & =d S d l
\end{aligned}
$$

On performing a few mathematical manipulation on the velocity field equation with the above three terms it leads to, (Ref. [14] pp. 38) 


$$
\vec{V}=\frac{1}{4 \pi} \int_{Q} \frac{\vec{\zeta} \times\left(\overrightarrow{r_{0}}-\overrightarrow{r_{1}}\right)}{\left|\overrightarrow{r_{0}}-\overrightarrow{r_{1}}\right|^{3}} d Q
$$

\subsubsection{Velocity Induced by a Straight Vortex Section}

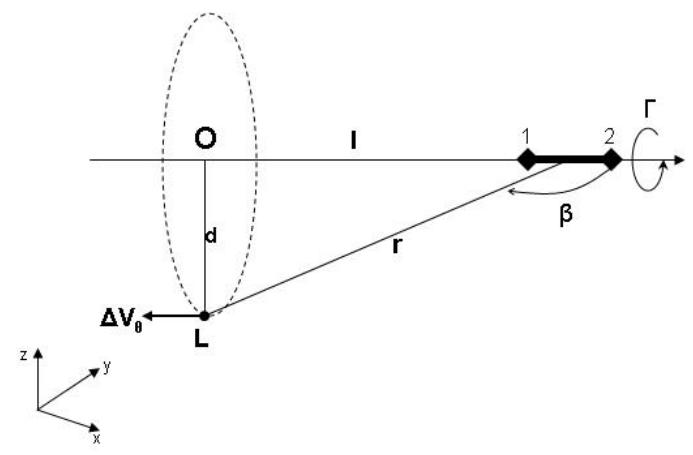

Figure 2.4: Velocity Induced By A Straight Vortex Segment

The velocity induced by a straight vortex section is derived from the Biot-Savart's law. The vortex segment is considered to be a part of a continuous vortex line. The vortex segment has a constant circulation $\Gamma$, and induces a tangential velocity, as shown in Figure 2.4. The velocity induced by this vortex segment at a point that is a distance $\mathbf{r}$ away from it is given by,

$$
\Delta \vec{V}=\frac{\Gamma}{4 \pi} \frac{d \mathbf{l} \times \vec{r}}{r^{3}}
$$




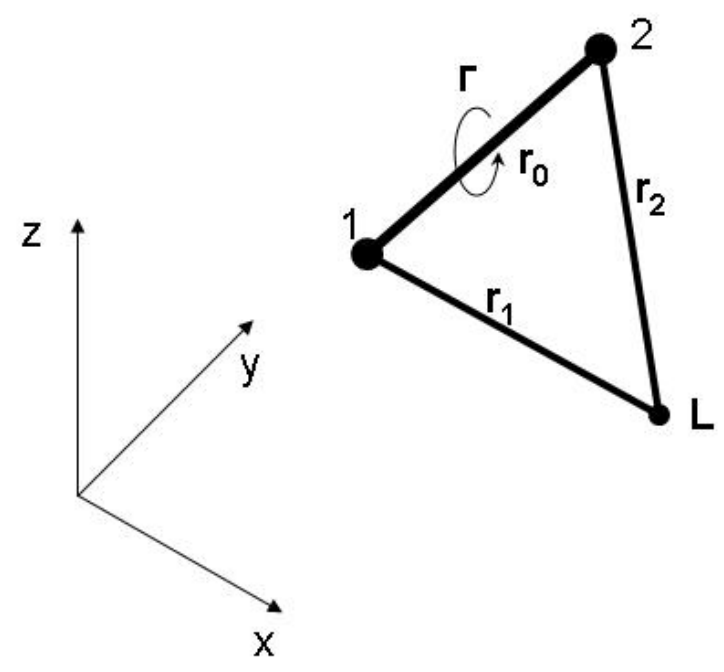

Figure 2.5: Nomenclature used for the velocity induced by a three-dimensional, straight vortex segment

By performing geometric manipulations and using basic trigonometric functions, as illustrated in Katz and Plotkin (Ref. [14] pp. 39), the velocity induced by the section of the vortex line can be written as,

$$
\vec{V}=\frac{\Gamma}{4 \pi} \frac{\overrightarrow{r_{1}} \times \overrightarrow{r_{2}}}{\left|\overrightarrow{r_{1}} \times \overrightarrow{r_{2}}\right|^{2}} \overrightarrow{r_{0}} \cdot\left(\frac{\overrightarrow{r_{1}}}{\left|\overrightarrow{r_{1}}\right|}-\frac{\overrightarrow{r_{2}}}{\left|\overrightarrow{r_{2}}\right|}\right)
$$

where $\overrightarrow{r_{1}}$ is the distance from point 1 to point $\mathrm{L}, \overrightarrow{r_{1}}$ is the distance from point 2 to point L, and $\overrightarrow{r_{0}}$ describes the vortex segment, as shown in Fig. 2.5 . 


\subsection{Vortex Lattice Method}

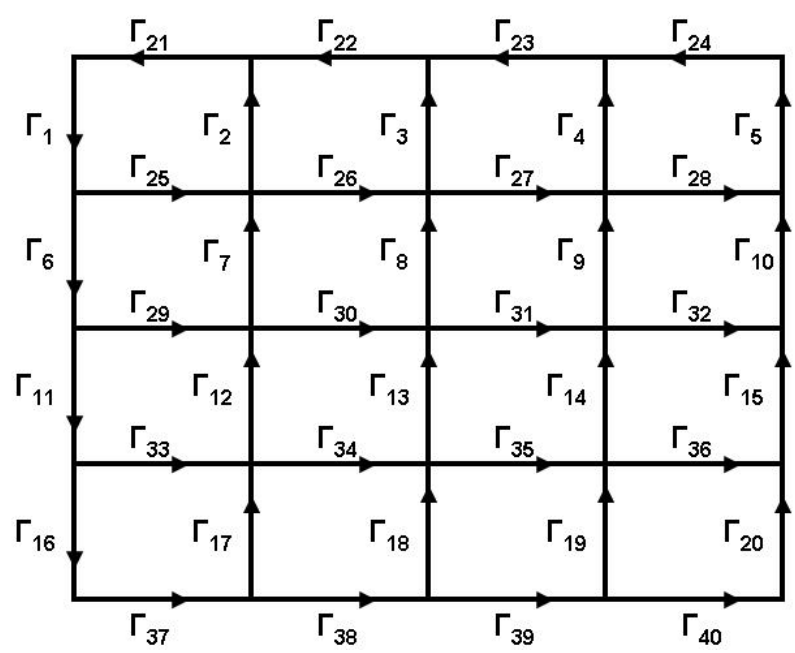

Figure 2.6: Chordwise And Spanwise Running Vortex Segments

The vortex lattice method used in this work is based on references [20] and [7]. The vortex lattice consists of vortex lines that run along the span wise and chord wise direction of the wing, see Figure 2.6. Biot-Savart law serves as the basis for the calculation of velocity. In conjunction with a few theorems and boundary conditions, it can be effectively used for the prediction of aerodynamic loads and the wake parameters.

\subsubsection{Spatial Conservation of Vorticity}

Consider at any instant, a surface $S$ that encloses a region $R$. The application of divergence theorem yields, 


$$
\int_{S} \vec{\zeta} \cdot \vec{n} d S=\int_{R} \vec{\nabla} \cdot \vec{\zeta} d Q=0
$$

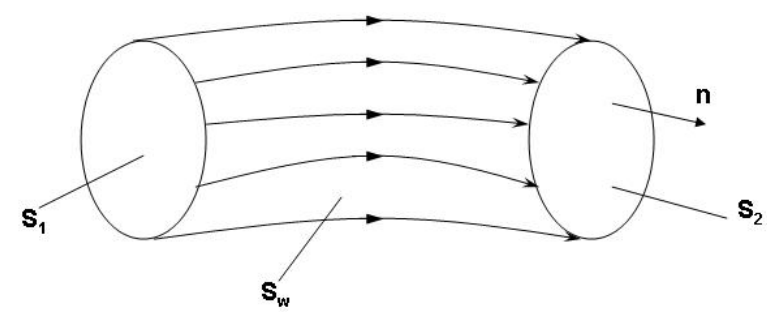

Figure 2.7: Vortex Tube

An application of this principle to a tube, as shown in Figure 2.7. The tube is enclosed by circular surfaces $S_{1}$ and $S_{2}$, and along its length by surface $S_{w}$. Since the vorticity is parallel to the surface $S_{w}$, its contribution vanishes resulting in,

$$
\int_{S} \vec{\zeta} \cdot \vec{n} d S=\int_{S_{1}} \vec{\zeta} \cdot \vec{n} d S+\int_{S_{2}} \vec{\zeta} \cdot \vec{n} d S=0
$$

The normal vector in the equation is the outward normal. Denoting $n_{v}$ as being positive and in the direction of the vorticity vector, the equation becomes, 


$$
\int_{S_{1}} \vec{\zeta} \cdot \overrightarrow{n_{v}} d S=\int_{S_{2}} \vec{\zeta} \cdot \overrightarrow{n_{v}} d S=\text { const }
$$

The circulation of a curve $C$ that surrounds the tube, and lies on its wall is given by,

$$
\Gamma_{C}=\int_{S} \vec{\zeta} \cdot \overrightarrow{n_{v}} d S=\text { const }
$$

\subsubsection{Helmholtz's theorem}

The application of the above equation to a vortex filament yields,

$$
\Gamma_{C}=\vec{\zeta} d S=\text { const }
$$

Helmholtz's theorem deals with the rate of change of vorticity. Helmholtz vortex theorems can be summarized as:

1. The strength of a vortex filament is constant along its length. 
2. A vortex filament cannot start or end in a fluid. It must form a closed path or extend to infinity.

3. The fluid that forms a vortex tube continues to form a vortex tube and the strength of the vortex tube remains constant as the tube moves about. Hence, vortex elements such as vortex lines, vortex tubes, vortex surfaces, etc., will remain vortex elements with time.

\subsubsection{Kelvin's theorem}

Kelvin's Circulation theorem deals with the rate of change of circulation. The theorem states that, In the motion of an inviscid fluid, the rate of change of circulation around any fluid curve is permanently zero if the body forces are irrotational and if there is a single-valued pressure-density relation, or, equivalently under these conditions, the circulation around a fluid curve remains a constant for all times as the curve moves with the fluid. The conclusion of the theorem is mathematically written as,

$$
\frac{D}{D t} \Gamma_{C}=\frac{D}{D t} \iint_{S} \vec{\zeta} \cdot \overrightarrow{n_{v}} d S=0
$$




\subsubsection{Discretized Vortex Sheet}

The vortex sheet consists of vortex lines running along the span wise and chord wise directions on the surface of the wing. The chord wise and span wise running vortex lines cross each other forming quadrilateral vortex elements, as shown in Figures 2.6 and 2.9. These are called vortex rings. In the work done in this thesis, only rectangular elements are considered. Experience has shown that rectangular elements used on the surface of the wing in VLM are more efficient than elements of other shapes [20]. The sum of the circulation at any node on the vortex lattice is zero, as shown in Figure 2.8. This is analogous to Kirchoff's principle in Electrical theory.

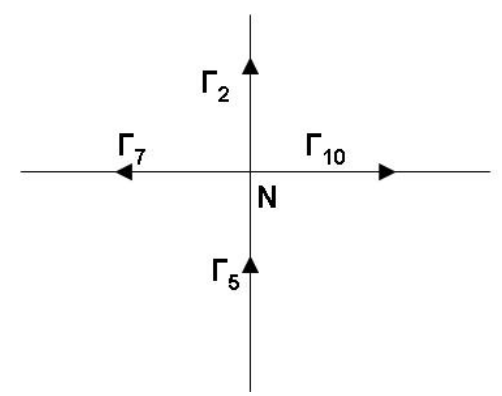

Figure 2.8: Sum of $\Gamma$ at a node is zero

The advantage of using vortex rings is that they simplify the programming [14]. The leading segment of the first row's vortex ring is placed on leading edge of the wing. Thus, the trailing edge of the last row's vortex ring lies on the trailing edge of the wing. The collocation or the control point is in the center of each element, and the normal to each element is defined at this point. 


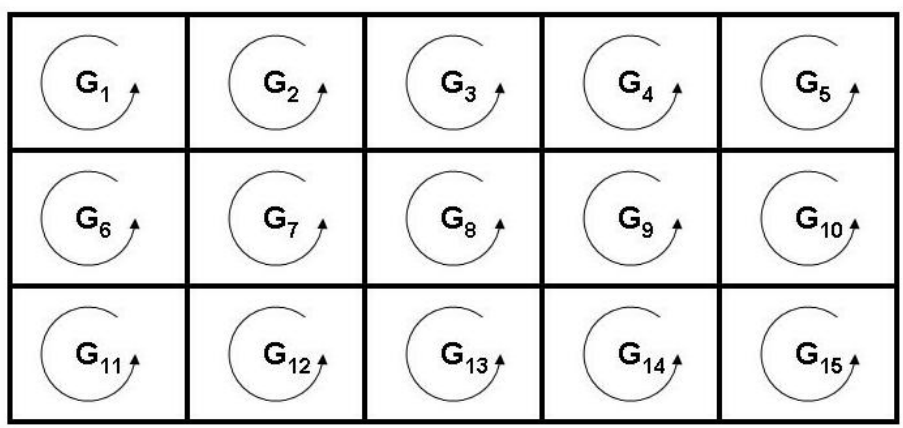

Figure 2.9: Rectangular Wing with Vortex Rings
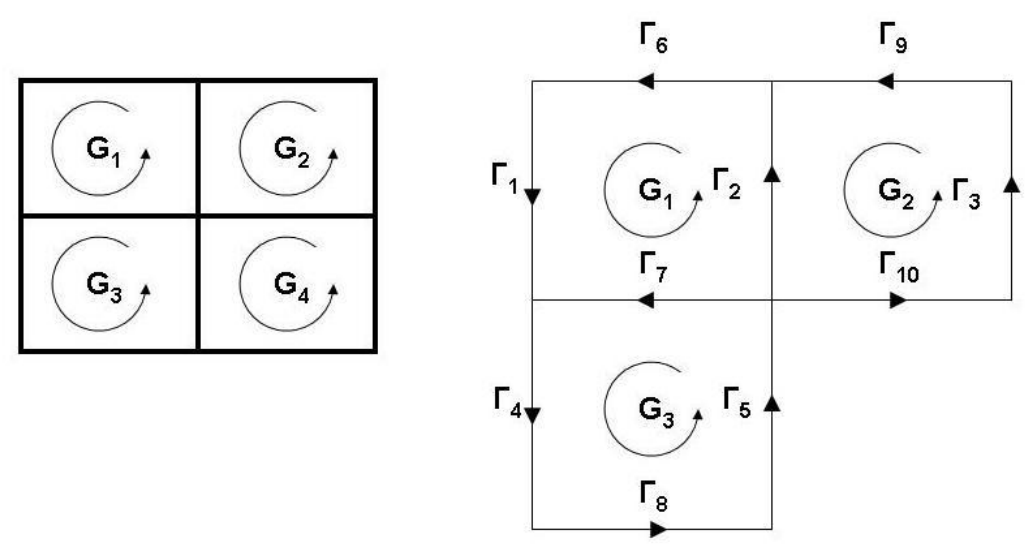

Figure 2.10: Relationship Between $G$ and $\Gamma$ 
Each vortex ring is defined to have a loop circulation which is defined by the letter $G_{i}$ for panel $i$, shown in Figure 2.9. It is defined positive in the counter-clockwise direction or in the positive $\vec{k}$ direction. The circulation of vortex segments lying on the leading edge and the wing tip will be the same as the vortex ring on which they lie. Thus from Figure 2.10 it can be deduced that,

$$
\begin{aligned}
& \Gamma_{1}=\Gamma_{6}=G_{1} \\
& \Gamma_{4}=\Gamma_{8}=G_{3} \\
& \Gamma_{3}=\Gamma_{9}=G_{2}
\end{aligned}
$$

The value of the circulation for a vortex segment that is an overlap of two different vortex segments is the difference in the values of the loop circulations of the two vortex rings involved.

$$
\begin{aligned}
& \Gamma_{7}=G_{1}-G_{3} \\
& \Gamma_{2}=G_{2}-G_{1}
\end{aligned}
$$

The circulation of the vortex segment lying on the trailing edge of the wing is the difference between the value of the last row of vortex rings at any time minus its value at the previous time. This is because a row of vortex rings is shed to conserve 


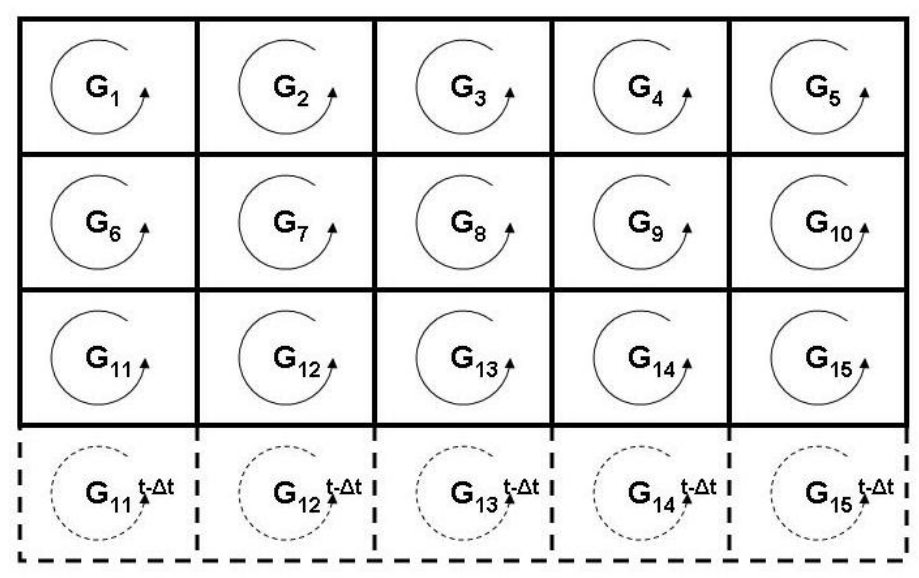

Figure 2.11: One Row of Shed Vortex Rings

the circulation, which is discussed in the next section, as shown in Figure 2.11. The same principle used in the previous two cases is used in the calculating the circulation of the vortex segments that lie on the trailing edge of the wing.

\subsubsection{Boundary conditions}

\section{No Penetration Condition}

Using the vortex ring method, the boundary conditions are satisfied on the wing surface, which can have camber and different planform shapes [14]. The no penetration condition implies that the fluid cannot pass through the wing. Another way of looking at the no penetration condition, is saying that the normal component of the structural velocity of the wing is equal to the normal component of the velocities 
induced by vortex panels on the surface of the wing and in the wake. This condition is applied at the control point of each element, which is the center of the element. The velocities acting on the wing at the control points originate from the freestream, structural dynamics, and the induced wake velocity. This is mathematically written as,

$$
\left(\vec{V}_{s}+\vec{V}_{\infty}\right) \cdot \vec{n}=\left(\vec{V}_{\text {wake }}+\vec{V}_{\text {surf }}\right) \cdot \vec{n}
$$

In the equation above, $V_{s}$ is the velocity of the wing structure, and can be written as,

$$
\vec{V}_{s}=\vec{V}_{\text {rigid }}+\vec{V}_{\text {membrane }}
$$

where, $\vec{V}_{\text {membrane }}$ is the velocity due to structural deformations

$\vec{V}_{\text {rigid }}$ is the velocity with which the wing moves, and is described for an oscillating plunge $h=h_{0} \cos \omega_{0} t$ and plunge $\alpha=\alpha_{0} \cos \left(\omega_{0} t+\phi\right)$ as, 


$$
\vec{V}_{\text {rigid }}=\overrightarrow{\dot{h}}+\overrightarrow{\dot{\alpha}} \times \vec{R}_{a}
$$

where, $\overrightarrow{R_{a}}$ is the distance arm from the pitching axis to the control point.

$\vec{V}_{\text {surf }}$ is the velocity induced on the control points by the vortex rings on the surface of the wing. $\vec{n}$ is the unit normal vector of the element on which the condition is applied. $\vec{V}_{\text {wake }}$ is the velocity induced by vortices in the wake on the control points on the wing.

$\vec{V}_{\text {wake }}$ and $\vec{V}_{\text {surf }}$ are both calculated using the Biot-Savart's law for the vortex ring. The velocity from the structures is elaborated in the next chapter. The loop circulation on the surface at any given time is computed based on the structural and the wake velocity from the previous time step. Thus, the problem involves solving for a number of unknowns which is equal to the number of surface elements.

$$
\begin{aligned}
A_{i j} G_{j} & =V_{\text {surf }} \\
V_{\text {surf }} & =\left(\vec{V}_{\infty}+\vec{V}_{s}-\vec{V}_{\text {wake }}\right) \cdot \vec{n}
\end{aligned}
$$

where A is the coefficient matrix consisting the coefficients from the Biot-Savart law. 


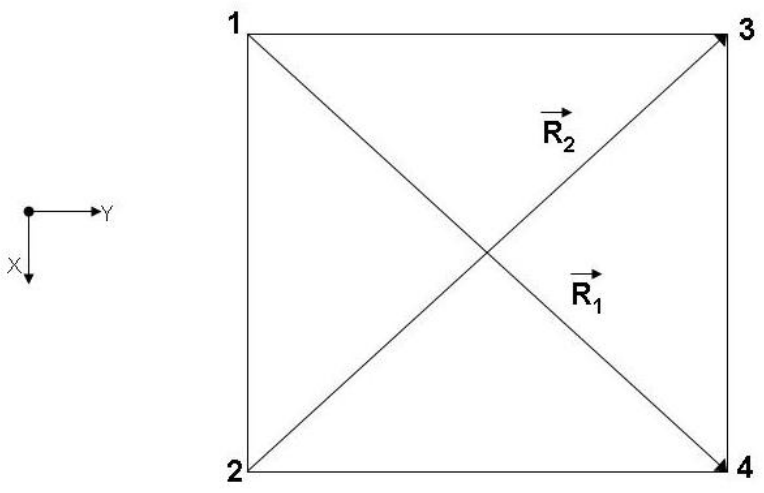

Figure 2.12: Calculation of Normal Vector

In the equation above, $i$ is the panel on which the normal velocity is being computed, and $j$ is the index of the panel loop circulation that induces the velocity. The unit out ward normal is computed by the cross product of vectors connecting nodes diagonally across each other, as shown in Figure 2.12.

$$
\begin{aligned}
\vec{n} & =\frac{\overrightarrow{R_{1}} \times \overrightarrow{R_{2}}}{\left|\overrightarrow{R_{1}} \times \overrightarrow{R_{2}}\right|} \\
\overrightarrow{R_{1}} & =\left(x_{4}, y_{4}, z_{4}\right)-\left(x_{1}, y_{1}, z_{1}\right) \\
\overrightarrow{R_{2}} & =\left(x_{3}, y_{3}, z_{3}\right)-\left(x_{2}, y_{2}, z_{2}\right)
\end{aligned}
$$




\section{Kutta Condition and Vortex Convection}

The Kutta condition is imposed by shedding the vortices from the trailing edge of the wing. By implementing the Kutta condition at the trailing edge, it is being assumed that the flow separates at the trailing edge. The velocity induced on the wake nodes is computed by the Biot-Savart law. Each node in the wake experiences velocities from the freestream, the vortex panels on the surface and vortex panels in the wake. The wake nodes travel a distance that is equal to the velocity times the time interval. The wake panels contain constant circulation, which is acquired during the shedding process from the last row of panels on the wing's surface. The shedding and convection process generates the wake, which induces velocity on the control points at the next time step. The process of the formation of new vortices, and convection from the trailing edge continues for any desired number of time steps. From the nature of the wake formation process, the wake keeps a history of the circulation strength. From the Biot-Savart law, the velocity induced on a point by a vortex panel is inversely proportional to the distance. Thus, the vortices far away from the wing have negligible effect on the aerodynamic loads. Many VLM codes cut off the vortices that have traveled far from the wing. Typically, a vortex segment that has traveled a distance of 8 to 10 chord lengths has negligible effect on the aerodynamic loads. 


\subsubsection{Calculation of Load Coefficient}

The formulation of the load coefficient is acquired from Ref.[7]. The pressure coefficient is calculated by the mathematical manipulation of Bernoulli's equation. Bernoulli's equation for incompressible unsteady flow is given by,

$$
\begin{aligned}
\frac{\partial \phi}{\partial t}+\frac{1}{2} \nabla \phi \cdot \nabla \phi+\frac{p}{\rho} & =P_{f s} \\
P_{f s} & =\frac{1}{2} V_{\infty}^{2}+\frac{p_{\infty}}{\rho}
\end{aligned}
$$

In the equation above, $p$ is the pressure, $\rho$ is the density is the density of air, $P_{f s}$ is the far stream stagnation pressure calculated at conditions at infinity, $p_{\infty}$ is the freestream pressure, $V_{\infty}$ is the freestream velocity, and $\phi$ is the scalar gradient of the velocity. The pressure coefficient $C_{p}$ is derived as shown below,

$$
\begin{aligned}
\frac{p}{\rho}-\frac{p_{\infty}}{\rho} & =\frac{1}{2} V_{\infty}^{2}-\frac{1}{2} \nabla V \cdot \nabla V-\frac{\partial \phi}{\partial t} \\
& =\frac{1}{2} V_{\infty}^{2}\left[1-\frac{V}{V_{\infty}}-\frac{2}{V_{\infty}^{2}} \frac{\partial \phi}{\partial t}\right] \\
\frac{p-p_{\infty}}{\rho} & =\frac{1}{2} V_{\infty}^{2}\left[1-\frac{V}{V_{\infty}}-\frac{2}{V_{\infty}^{2}} \frac{\partial \phi}{\partial t}\right] \\
C_{p} & =\frac{p-p_{\infty}}{\frac{1}{2} \rho V_{\infty}^{2}}=1-\frac{V}{V_{\infty}}-\frac{2}{V_{\infty}^{2}} \frac{\partial \phi}{\partial t}
\end{aligned}
$$


In order to calculate the loads on the wing, the difference in pressure across the wing is essential. Thus, the next parameter that is calculated is $\Delta C_{p}$. The parameter is derived by nondimensionalizing the velocity, the scalar gradient of the velocity, and the time.

$$
\tilde{V}=\frac{V}{V_{\infty}}, \tilde{\phi}=\frac{\phi}{V_{\infty} L_{p}}, \tilde{t}=\frac{t}{L_{p} V_{\infty}}
$$

where, $\tilde{V}, \tilde{\phi}$, and $\tilde{t}$ are the nondimensional velocity, scalar of the velocity gradient, and time respectively. The velocity is nondimensionalized with respect to the freestream velocity $V_{\infty}$ for convenience. $L_{p}$ is the length of a panel in the chord wise direction.

$$
L_{p}=\frac{c}{n_{c p}}
$$

where $c$ is the chord length, and $n_{c p}$ is the number of chord wise panels.

The time step is assigned a value such that the nondimensional time step has a value of one. By taking this step, the bound vortex elements and the wake vor- 
tex elements have the same size. Experience has shown same size vortex elements are better than vortex elements of different sizes. After nondimensionalizing, the equation describing $C_{p}$ has the following form,

$$
C_{p}=1-\tilde{V}^{2}-2 \frac{\partial \tilde{\phi}}{\partial \tilde{t}}
$$

Since the no penetration condition is applied at the control points of the element, the aerodynamic pressure coefficient is also calculated at the same point. This is computed by taking the difference between the pressure coefficient computed on the lower surface and the upper surface of the wing.

$$
\begin{aligned}
\Delta C_{p} & =\left(C_{p}\right)_{L}-\left(C_{p}\right)_{U} \\
& =\left(\tilde{V}_{U}\right)^{2}-\left(\tilde{V}_{L}\right)^{2}+2\left(\left.\frac{\partial \tilde{\phi}}{\partial \tilde{t}}\right|_{U}-\left.\frac{\partial \tilde{\phi}}{\partial \tilde{t}}\right|_{L}\right) \\
& =\vec{V}_{U} \cdot \vec{V}_{U}+\overrightarrow{\tilde{V}}_{L} \cdot \vec{V}_{L}+2\left(\left.\frac{\partial \tilde{\phi}}{\partial \tilde{t}}\right|_{U}-\left.\frac{\partial \tilde{\phi}}{\partial \tilde{t}}\right|_{L}\right)
\end{aligned}
$$

where, $\tilde{V}_{U}$ is the local particle velocity at a point just over the lattice, $\tilde{V}_{L}$ is the 


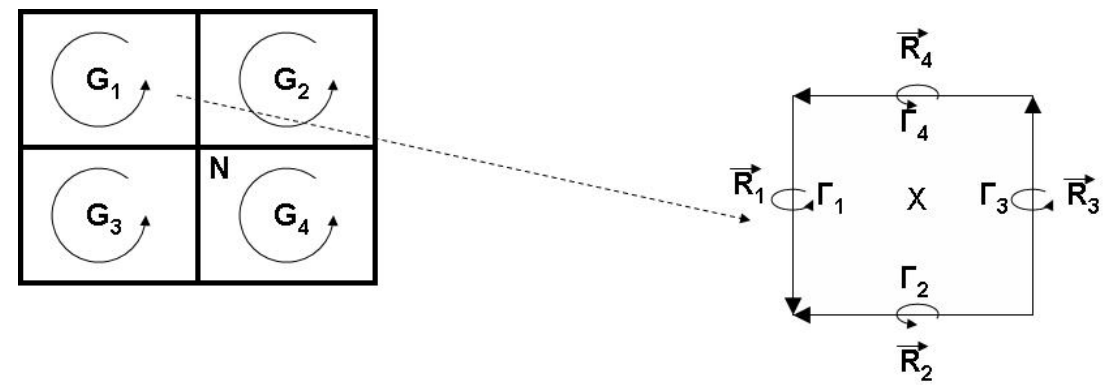

Figure 2.13: Anatomy of a vortex element

local particle velocity at a point under over the lattice, $\left.\frac{\partial \tilde{\phi}}{\partial \hat{t}}\right|_{U}$ and $\left.\frac{\partial \tilde{\phi}}{\partial t}\right|_{L}$ are evaluated on the upper and lower surfaces of the control point of the wing respectively.

The calculation of $\Delta C_{p}$ is based on the calculation of $\tilde{V}$ and $\frac{\partial \tilde{\phi}}{\partial t}$ on the upper and lower surfaces.

$$
\begin{aligned}
& \overrightarrow{\tilde{V}}_{U}=\overrightarrow{\vec{V}_{a}}+\frac{\Delta \overrightarrow{\tilde{V}}}{2} \\
& \overrightarrow{\tilde{V}}_{L}=\overrightarrow{\tilde{V}}_{a}-\frac{\Delta \overrightarrow{\tilde{V}}}{2}
\end{aligned}
$$

where $\vec{V}_{a}$ is the sum of all the velocities induced by the bound surface vortices and wake vortices, and the freestream velocity. $\Delta \overrightarrow{\tilde{V}}$ denotes the jump in the tangential velocity across the vortex sheet, which is equal to the vortex sheet strength. For the derivation of $\Delta \overrightarrow{\tilde{V}}$, a new vector $\overrightarrow{\tilde{\Gamma}}$ is defined, whose components are shown in Figure 2.13 


$$
\begin{aligned}
\overrightarrow{\tilde{\Gamma}} & =\frac{1}{2}\left(\overrightarrow{\Gamma_{1}}+\overrightarrow{\Gamma_{2}}+\overrightarrow{\Gamma_{3}}+\overrightarrow{\Gamma_{4}}\right) \\
\overrightarrow{\Gamma_{i}} & =\Gamma_{i} \frac{R_{i}}{r_{i}}
\end{aligned}
$$

where, $r_{i}=\left|R_{i}\right|$.

$\Delta \overrightarrow{\tilde{V}}$ is thus defined by,

$$
\begin{aligned}
\Delta \overrightarrow{\tilde{V}} & =-\frac{\vec{n} \times \overrightarrow{\tilde{\Gamma}}}{\tilde{A}} \\
\tilde{A} & =\frac{1}{2}\left(\frac{\overrightarrow{R_{1}} \times \overrightarrow{R_{3}}}{\left|\overrightarrow{R_{1}} \times \overrightarrow{R_{3}}\right|}+\frac{\overrightarrow{R_{2}} \times \overrightarrow{R_{4}}}{\left|\overrightarrow{R_{2}} \times \overrightarrow{R_{4}}\right|}\right)
\end{aligned}
$$

Next, $\frac{\partial \tilde{\phi}}{\partial \tilde{t}}$ is evaluated. The time derivative of the velocity potential, at a given point, is mathematically defined as,

$$
\frac{\partial \phi(r, t)}{\partial t}=\lim _{\Delta t \rightarrow 0} \frac{\phi(r, t+\Delta t)-\phi(r, t)}{\Delta t}
$$

where $\vec{r}$ is the position vector in the inertial frame.

The distance traveled by a control point, denoted by point $Y$ and $Y^{\prime}$ at time $t$ 


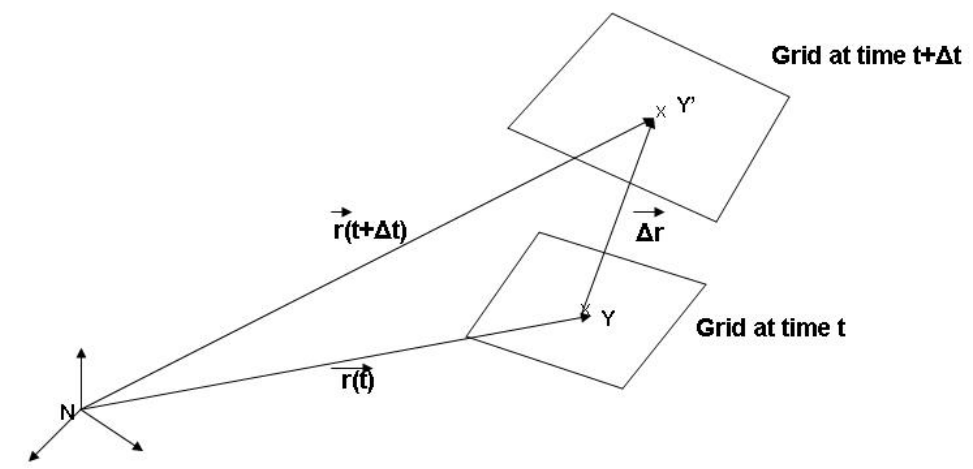

Figure 2.14: Description of Grid at times $t$ and $t+\Delta t$

and $t+\Delta t$ respectively is described mathematically by $\Delta r$. This is illustrated in Figure 2.14 .

$$
\overrightarrow{\Delta r}=\vec{r}(t+\Delta t)-\vec{r}(t)
$$

The velocity potential at time $t+\Delta t$ can be expressed by the expansion of Taylor series. Thus,

$$
\begin{aligned}
\phi(\vec{r}+\Delta \vec{r}, t+\Delta t) & =\phi(\vec{r}, t+\Delta t)+\nabla \phi(\vec{r}, t+\Delta t) \cdot \Delta \vec{r}+\ldots \\
\phi(\vec{r}, t+\Delta t) & \approx \phi(\vec{r}+\Delta \vec{r}, t+\Delta t)-\nabla \phi(\vec{r}, t+\Delta t) \cdot \Delta \vec{r}
\end{aligned}
$$

Eq. 2.58 can be expanded using the Taylor series expansion and thereby be 
written as,

$$
\begin{aligned}
\frac{\partial \phi(r, t)}{\partial t} & =\lim _{\Delta t \rightarrow 0} \frac{\phi(\vec{r}+\Delta \vec{r}, t+\Delta t)-\phi(\vec{r}, t)-\nabla \phi(\vec{r}, t+\Delta t) \cdot \Delta \vec{r}}{\Delta t} \\
& =\lim _{\Delta t \rightarrow 0} \frac{\phi(\vec{r}+\Delta \vec{r}, t+\Delta t)-\phi(\vec{r}, t)}{\Delta t}-\lim _{\Delta t \rightarrow 0} \nabla \phi(\vec{r}, t+\Delta t) \cdot \frac{\Delta \vec{r}}{\Delta t}(2 .
\end{aligned}
$$

The body velocity at point $L$ fixed at a vortex lattice is given by $\lim _{\Delta t \rightarrow 0} \frac{\Delta \vec{r}}{\Delta t}$. Thus,

$$
\begin{aligned}
\frac{\partial \phi(r, t)}{\partial t} & =\lim _{\Delta t \rightarrow 0} \frac{\phi(\vec{r}+\Delta \vec{r}, t+\Delta t)-\phi(\vec{r}, t)}{\Delta t}-\nabla \phi(\vec{r}, t+\Delta t) \cdot \overrightarrow{V_{B L}} \\
& =\left.\frac{D \phi}{D t}\right|_{L}-\nabla \phi(\vec{r}, t+\Delta t) \cdot \overrightarrow{V_{B L}}
\end{aligned}
$$

where $\frac{D \phi}{D t}$ is the total derivative of $\phi(\vec{r}, t)$. Non dimensionalizing the parameters in the equation above yields,

$$
\begin{aligned}
\frac{\partial \tilde{\phi}}{\partial \tilde{t}} & =\frac{D \tilde{\phi}}{D \tilde{t}}-\frac{t}{V_{\infty} L_{p}} V_{\infty}^{2} \tilde{\nabla}(\tilde{\phi}) \cdot \overrightarrow{\tilde{V_{B L}}} \\
& =\frac{D \tilde{\phi}}{D \tilde{t}}-\tilde{\nabla}(\tilde{\phi}) \cdot \overrightarrow{V_{B L}}
\end{aligned}
$$


$V_{\infty}=L_{p} / \Delta t$

For the upper and lower surfaces $\frac{\partial \tilde{\phi}}{\partial \tilde{t}}$ and their difference is written as,

$$
\begin{array}{r}
\left.\frac{\partial \tilde{\phi}}{\partial \tilde{t}}\right|_{U}-\left.\frac{\partial \tilde{\phi}}{\partial \tilde{t}}\right|_{L}=\frac{D}{D \tilde{t}}\left[\tilde{\phi}\left(\vec{r}_{U}, t\right)-\tilde{\phi}\left(\vec{r}_{L}, t\right)\right] \\
-\left[\tilde{\nabla}\left(\tilde{\phi}\left(\overrightarrow{r_{U}}, t\right)\right)-\tilde{\nabla}\left(\tilde{\phi}\left(\overrightarrow{r_{L}}, t\right)\right)\right] \cdot \overrightarrow{V_{B L}}
\end{array}
$$

Now,

$$
\begin{aligned}
& \tilde{\nabla}\left(\tilde{\phi}\left(\vec{r}_{U}, t\right)\right)=\overrightarrow{\tilde{V}}_{U}=\overrightarrow{\tilde{V}}_{a}+\frac{\Delta \overrightarrow{\tilde{V}}_{U}}{2} \\
& \tilde{\nabla}\left(\tilde{\phi}\left(\vec{r}_{L}, t\right)\right)=\overrightarrow{\tilde{V}}_{L}=\overrightarrow{\vec{V}}_{a}-\frac{\Delta \overrightarrow{\tilde{V}}_{U}}{2}
\end{aligned}
$$

This yields,

$$
\tilde{\nabla}\left(\tilde{\phi}\left(\overrightarrow{r_{U}}, t\right)\right)-\tilde{\nabla}\left(\tilde{\phi}\left(\overrightarrow{r_{L}}, t\right)\right)=\Delta \overrightarrow{\vec{V}}
$$

Moreover from Eq. 2.25, $\tilde{\nabla}\left(\tilde{\phi}\left(\overrightarrow{r_{U}}, t\right)\right)-\tilde{\nabla}\left(\tilde{\phi}\left(\overrightarrow{r_{L}}, t\right)\right)$ can be calculated by, 


$$
\left.\left(\tilde{\phi}\left(\overrightarrow{r_{U}}, t\right)\right)-\left(\tilde{\phi}\left(\overrightarrow{r_{L}}, t\right)\right)=\oint \tilde{\vec{V}} \tilde{d r}=\Gamma \tilde{(} t\right)=-G(t)
$$

Using a first order finite-difference $\frac{D \tilde{G}_{i}}{D \tilde{t}}$ can be approximated, for panel $i$, as

$$
\frac{D \tilde{G}_{i}}{D \tilde{t}}=\frac{\tilde{G}_{i}(t)-\tilde{G}_{i}(t-\Delta t)}{\Delta t}
$$

Thus, the element force vectors is given by,

$$
F(i, j)=\frac{1}{2} \rho U_{\infty}^{2} L_{p} \Delta C_{p}(i, j) L_{p}^{2} n(\vec{i}, j)
$$

The lift and induced drags can be calculated by taking the component of the forces in the $z$ and freestream $x$ directions respectively.

$$
\begin{aligned}
L & =F \cdot \overrightarrow{n_{z}} \\
D & =F \cdot \overrightarrow{n_{x}}
\end{aligned}
$$




\subsubsection{Implementation of VLM}

The vortex lattice sheet is discretized on the surface of the wing with $n r$ and $n c$ number of nodes in rows and columns respectively. The vortices running in the chordwise and spanwise direction cross each other at these nodes forming vortex rings as seen in Figure 2.9. These vortex rings thus have vortex segments on its boundaries. These vortex rings, composed of finite vortex segments, can induce velocity on any point in space using the Biot-Savart law. At any given time, the Biot-Savart law is used to compute the induced velocity by these bound vortex rings, on the control points. It is also used to compute the induced velocity on the nodes at the trailing edge and in the wake.

The velocity with which the trailing edge is shed into the wake is the sum of the velocity induced by the bound vortex rings and the wake vortex rings, and the freestream velocity. The time step $d t$ is pre-set to a value such that in a time step the trailing edge travels a distance that is equal to the chord length of a panel, as seen in Figure 2.11. This makes sure that the wake vortex rings and the bound rings are about the same size.

The following steps outline the aerodynamic modeling.

- The boundary condition is satisfied on each control point on the surface of the wing. The boundary condition is given by, 


$$
\left(\vec{V}_{s}+\vec{V}_{\infty}\right) \cdot \vec{n}=\left(\vec{V}_{\text {wake }}+\vec{V}_{\text {surf }}\right) \cdot \vec{n}
$$

where,

$$
\vec{V}_{s}=\vec{V}_{\text {rigid }}+\vec{V}_{\text {membrane }}
$$

- The individual velocity components can be elaborated as,

$$
\begin{aligned}
\vec{V}_{\text {rigid }} & =\overrightarrow{\dot{h}}+\overrightarrow{\dot{\alpha}} \times \vec{R}_{a} \\
A_{\text {ij }} G_{j} & =V_{\text {surf }} \\
V_{\text {surf }} & =\left(\vec{V}_{\infty}+\vec{V}_{s}-\vec{V}_{\text {wake }}\right) \cdot \vec{n}
\end{aligned}
$$

where $A_{i j}$ is the coefficient matrix that contains the influence of bound vortex $j$ on control point $i$ through the Biot-Savart method, as seen in Eq. 2.21. The normal vector is computed as shown in Eq. 2.40. At the first time step, the wake does not contain any wake panels, thus there is no velocity induced by the wake vortex rings, $\vec{V}_{\text {wake }}$, on the control points. Also, the velocity due to the membrane does not exist, as the wing is set into motion from an undeformed 
state.

- On satisfying the boundary condition, the vortex ring strengths are computed. Using this vortex ring strength, the velocity on the wake nodes, which comprises the trailing edge nodes, is computed. The wake elements are then moved in space for the next time step. The newly shed wake row assumes the value of the last row of vortex rings lined up along the span of the wing. It thereby keeps the history of the circulation of the previous time step.

- Now that the wake node locations are known for the new time step, the velocity induced by these wake ring elements is computed on the control points using the Biot-Savart law. The boundary condition on the control points can be satisfied and the new bound vortex ring strengths can be computed. The bound vortex ring strengths can be used to compute the pressure coefficients, and thus the lift coefficients.

\subsection{Theoretical Results}

In this section, the theoretical results used in the validation of the aerodynamic loads are described. For the cases of a very high aspect ratio wing pitch and plunge, Theodorsen's theoretical results are used for comparison [21]. Theodorsen's ana- 
lytical results for the vortex strengths, and lift for plunge and pitch described by, $h=h_{0} e^{i \omega t}$ and $\alpha=\alpha_{0} e^{i \omega t}$ respectively are given by,

$$
\begin{aligned}
\Gamma_{T} & =\frac{4 b i}{k} e^{-i k}\left[H(k)\left(V_{\infty} \alpha+\dot{h}+\frac{1}{2} b \dot{\alpha}\right)\right] \\
L_{T} & =2 \pi \rho b\left[\left(\frac{b}{2}\right)\left(V_{\infty} \dot{\alpha}+\ddot{h}-b a \ddot{\alpha}\right)+C(k) V_{\infty}\left(V_{\infty} \alpha+\dot{h}+b\left(\frac{1}{2}-a\right) \dot{\alpha}(\$) .79\right)\right.
\end{aligned}
$$

where,

$$
\begin{aligned}
H(k) & =\frac{1}{H_{1}^{(2)}(k)+i H_{0}^{(2)}(k)} \\
C(k) & =\frac{H_{1}^{(2)}(k)}{H_{1}^{(2)}(k)+i H_{0}^{(2)}(k)} \\
k & =\frac{\omega b}{U_{\infty}}
\end{aligned}
$$

$h_{0}$ and $\alpha_{0}$ are the plunge and pitch amplitudes respectively that oscillate with a frequency of $\omega_{0}$ and $a$ is the distance between the midchord and the pitch axis nondimensionalized with respect to the semichord. $b$ is the semichord, and $V_{\infty}$ is the freestream velocity.

For a sudden change in angle of attack, and for a very high aspect ratio wing, Wagner's function is used for validation of the lift per unit span [21], which is given 
by,

$$
L_{w}=2 \pi \rho V_{\infty}^{2} b \alpha \phi(s)
$$

where,

$$
\begin{aligned}
s & =\frac{V_{\infty} t}{b} \\
\phi(s) & =\frac{2}{\pi} \int_{0}^{\infty} \frac{F(k)}{k} \sin (k s) d k=1+\frac{2}{\pi} \int_{0}^{\infty} \frac{G(k)}{k} \cos (k s) d k
\end{aligned}
$$

$F(k)$ and $G(k)$ are the real and imaginary parts of $C(k)$ respectively. 


\section{Chapter 3}

\section{Structural Modeling And}

\section{Aeroelasticity}

The wing is modeled as a membrane structure. A membrane is defined as a tensile structure, that can carry tension but cannot carry compression or have bending. Typical examples of membrane structures include a trampoline and a tent, where a membrane is supported by a rigid structure on the boundary. The pre-stress introduced into the membrane by stretching its boundaries give it a definite form and thereby enables its usage as a structure. Common materials used for building membranes are Teflon coated fibreglass, and PVC coated polyester. In one dimension, strings are an equivalent of membranes. 


\subsection{Membrane theory}

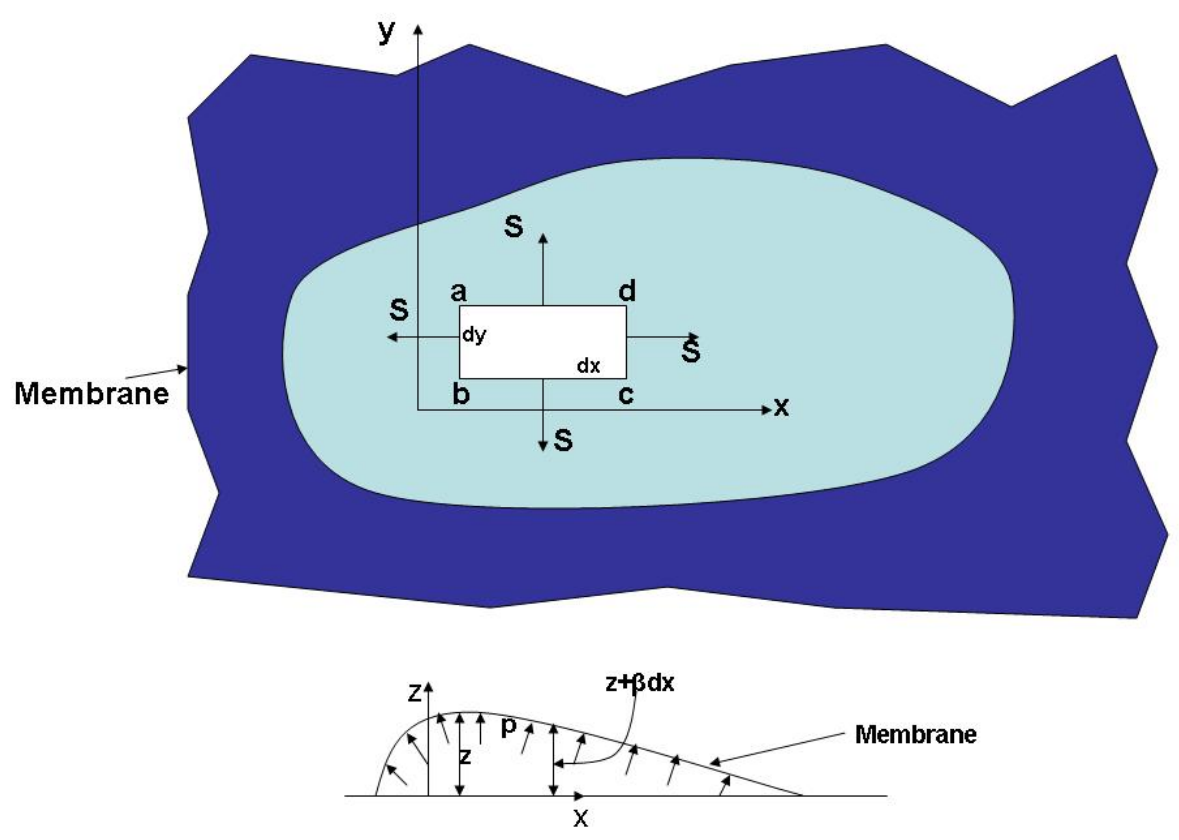

Figure 3.1: Description of Membrane And Membrane Deflection

Consider a homogenous membrane that is supported at the edge, given its boundary contour by a hole cut in a plate, as shown in Figure 3.1. The boundary of the membrane wing is rigid. The stress from the rigid boundary is introduced into the membrane wing, and is hereby referred to as the pre-stress as it is known before the system is set into a dynamical state. The membrane will have different displacements at different locations on the wing plane.

Consider the infintesimal element abcd in Figure 3.1. Let $S_{\text {stress }}$ be the tensile 
force per unit membrane length. From a small deflection $z, \beta \approx \frac{\partial z}{\partial x}$ describes the inclination of $S_{\text {stress }}$ acting on side $a b$. Since $z$ varies from point to point, the angle at which $S_{\text {stress }}$ is inclined on side $d c$ is given by,

$$
\beta+\frac{\partial \beta}{\partial x} d x=\frac{\partial w}{\partial x}+\frac{\partial^{2} w}{\partial x^{2}} d x
$$

where, $w$ is the deflection

Similarly, the inclination of the tensile forces are $\frac{\partial w}{\partial y}+\frac{\partial^{2} w}{\partial y^{2}} d y$, on sides $a d$ and $b c$ respectively. For constant $S_{\text {stress }}$, and uniform pressure $p$, the equation of a dynamically deforming membrane is given by,

$$
\begin{aligned}
&-\left(S_{\text {stress }} d y\right) \frac{\partial w}{\partial x}+S_{\text {stress }} d y\left(\frac{\partial z}{\partial x}+\frac{\partial^{2} w}{\partial x^{2}} d x\right)-\left(S_{\text {stress }} d x\right) \frac{\partial w}{\partial y} \\
&+\left(S_{\text {stress }} d x\right)\left(\frac{\partial w}{\partial y}+\frac{\partial^{2} w}{\partial y^{2}} d y\right)+p d x d y=\rho_{m} t_{w} \frac{\partial^{2} w}{\partial t^{2}} d x d y
\end{aligned}
$$

where $p$ is the pressure on the wing. Solving Eq. 3.2 leads to,

$$
\frac{\partial^{2} w}{\partial x^{2}}+\frac{\partial^{2} w}{\partial y^{2}}+\frac{p}{S_{\text {stress }}}=\frac{\rho_{m}}{S_{\text {stress }}} t_{w} \frac{\partial^{2} w}{\partial t^{2}}
$$


where, $S_{\text {stress }}$ is the pre-stress introduced into the membrane,

$\rho_{m}$ is the density of the membrane,

$t_{w}$ is the thickness of the wing

This is Poisson's equation [22], and the small slope restriction must be taken into account in view of the derivation.

\subsubsection{Static Membrane Deformation}

For a statically deforming membrane, the differential equation describing the deflection, in the wing plane is given replacing the right hand side of Eq. 3.2 by zero. Thus,

$$
\frac{\partial^{2} w}{\partial x^{2}}+\frac{\partial^{2} w}{\partial y^{2}}=-\frac{p}{S}
$$

where, $\rho_{m}$ is the density of the membrane.

\subsection{Application of Fourier Series}

For a geometry as shown in Figure 3.2 with simple supports along all edges, the solution to the membrane problem can be obtained by the application of Fourier 
series. The rectangular membrane can be thought of as a wing with rigid boundaries, with chord length $c$ and span $b$. The pressure and the structural deformations are thus represented by,

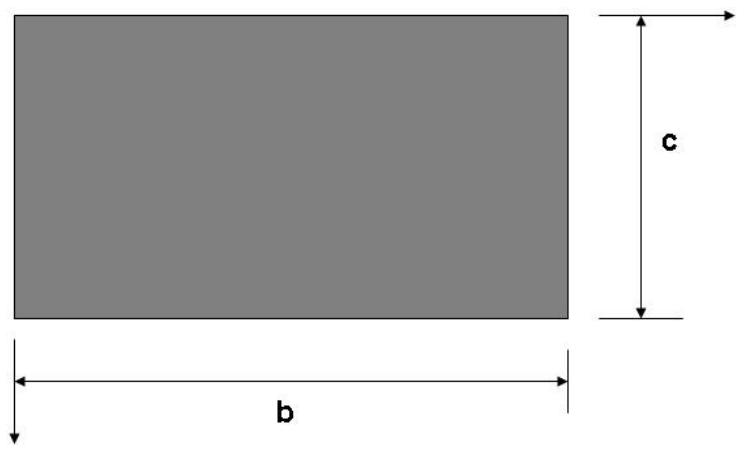

Figure 3.2: Rectangular Wing

$$
\begin{aligned}
& p(x, y)=\sum_{m=1}^{\infty} \sum_{n=1}^{\infty} p_{m n} \sin \frac{m \pi x}{c} \sin \frac{n \pi y}{b} \\
& w(x, y)=\sum_{m=1}^{\infty} \sum_{n=1}^{\infty} a_{m n} \sin \frac{m \pi x}{c} \sin \frac{n \pi y}{b}
\end{aligned}
$$

where $c$ and $b$ are the chord length and wing span respectively

Eq. 3.4 can be solved for $p_{m n}$ by using the following mathematical steps.

- Multiply both sides of the equation by $\sin \frac{m^{\prime} \pi x}{c} \sin \frac{n^{\prime} \pi y}{b}$ and integrate in $x$ and 


$$
\int_{0}^{b} \int_{0}^{c} p(x, y) \sin \frac{m^{\prime} \pi x}{c} \sin \frac{n^{\prime} \pi y}{b} d x d y=\int_{0}^{b} \int_{0}^{c} p_{m n} \sin \frac{m \pi x}{c} \sin \frac{n \pi y}{b} \sin \frac{m^{\prime} \pi x}{c} \sin \frac{n^{\prime} \pi y}{b} d x d y
$$

- Due to the orthogonality of the functions in the $x$ and $y$ directions; $m$ and $m$ ', and $n$ and n' have to be the same. Thus, it culminates at,

$$
\begin{gathered}
\int_{0}^{b} \int_{0}^{c} p(x, y) \sin \frac{m \pi x}{c} \sin \frac{n \pi y}{b} d x d y=\frac{b c}{4} p_{m n} \\
\Rightarrow p_{m n}=\frac{4}{b c} \int_{0}^{b} \int_{0}^{c} p(x, y) \sin \frac{m \pi x}{c} \sin \frac{n \pi y}{b} d x d y
\end{gathered}
$$

The pressure distribution is acquired from the aerodynamic computation of the loads and are represented discretely over the panels. The pressure distribution is represented by,

$$
p(x, y)=p(i, j)=\frac{1}{2} \rho U_{\infty}^{2} \Delta C_{p}(i, j)
$$


where $i$ and $j$ are the panel's row and column numbers respectively.

Each of the pressure coefficients can thus be represented by,

$$
p_{m n}=\left.\left.\sum_{i=1}^{i=n r-1} \sum_{j=1}^{j=n c-1} 4 \frac{p(i, j)}{m n} \cos \left(\frac{m \pi x}{c}\right)\right|_{x(i, j)} ^{x(i+1, j)} \cos \left(\frac{n \pi y}{b}\right)\right|_{y(i, j)} ^{y(i, j+1)}
$$

where $n c$ and $n r$ are the number of column and row nodes on the wing respectively. Once the pressure coefficients are computed, the coefficients for the structural deformations are computed. From this Fourier series approximations, the spatial and temporal partial differentials are,

$$
\begin{aligned}
\frac{\partial^{2} w}{\partial x^{2}} & =-\sum_{m=1}^{\infty} \sum_{n=1}^{\infty} a_{m n} \frac{m^{2} \pi^{2}}{c^{2}} \sin \frac{m \pi x}{c} \sin \frac{n \pi y}{b} \\
\frac{\partial^{2} w}{\partial y^{2}} & =-\sum_{m=1}^{\infty} \sum_{n=1}^{\infty} a_{m n} \frac{n^{2} \pi^{2}}{b^{2}} \sin \frac{m \pi x}{c} \sin \frac{n \pi y}{b} \\
\frac{\partial^{2} w}{\partial t^{2}} & =\sum_{m=1}^{\infty} \sum_{n=1}^{\infty} \ddot{a}_{m n} \sin \frac{m \pi x}{c} \sin \frac{n \pi y}{b}
\end{aligned}
$$

Plugging these approximations for the partial differentials into the differential equation for dynamic aeroelasticity Eq. 3.2 yeilds,

$$
-a_{m n} \pi^{2}\left(\frac{m^{2}}{c^{2}}+\frac{n^{2}}{b^{2}}\right)+\frac{p_{m n}}{S_{\text {stress }}}=\frac{\rho t_{w}}{S_{\text {stress }}} \ddot{a}_{m n}
$$




\subsection{Aeroelasticity}

Eq. 3.13 has two unknowns for each equation with a value for $m$ and $n$. The coupled problem is solved by approximating the unknowns using finite differencing methods. The section is divided into two parts, one for the aeroelasticity at the first time step and the other for all time steps after that.

\subsubsection{Time $t=t_{1}$}

The rectangular wing being studied is assumed to be at rest at $t=t_{0}$. There are no initial deformations on the membrane wing. At this time step, it has forward velocity $V_{\infty}$ and prescribed motion either in the form of an angle of attack, or plunge, or both. The following steps are taken to solve the aeroelastic problem.

- The wing moves with a pitching and plunging velocity. Since there are no structural deformation there is no velocity due to membrane structural deformation. The only velocity acting on the control points is that of the freestream.

- Using the no penetration condition on the control points on the wing as described in Eq. 2.77 and Eq. 2.37, the vortex ring strengths are computed.

- The vortex strengths are used to compute the pressure on the surface of the wing. 
- The bound vortex strengths computed in this time step induce a velocity on the trailing edge of the wing. This velocity along with the freestream velocity moves the vortex segments on the trailing edge into the wake for the next time step. The row of vortex panels shed have the ring strengths of the vortex rings on the last chordwise row of the wing. This is where the computations end at the first time step. The wake position required for the next time step is thus based on the wake velocities calculated at the present time step.

\subsubsection{Time $t_{i}>t_{1}$}

At this point we know the pressure at time $t=t_{i}-\Delta t$, and the wake position for $t=t_{i}$

- At time $t_{i}$, the aerodynamic pressure on the surface of the wing is used to compute the structural pressure coefficients in Eq. 3.9.

- The pressure coefficients computed are then used to compute the structural coefficients in Eq. 3.13. The structural coefficients are solved by the expansion of Eq. 3.13 as follows,

$$
-a_{m n}^{t_{i}-\Delta t} \pi^{2}\left(\frac{m^{2}}{c^{2}}+\frac{n^{2}}{b^{2}}\right)+\frac{p_{m n}^{t_{i}-\Delta t}}{S_{\text {stress }}}=\frac{\rho t_{w}}{S_{\text {stress }}} \ddot{a}_{m n}^{t_{i}-\Delta t}
$$


The second derivative of the structural coefficients is computed using central difference and represented by,

$$
\ddot{a}_{m n}^{t_{i}-\Delta t}=\frac{a_{m n}^{t_{i}}-2 a_{m n}^{t_{i}-\Delta t}+a_{m n}^{t_{i}-2 \Delta t}}{\Delta t^{2}}
$$

It needs to be pointed out that the central difference scheme is $2^{\text {nd }}$ order accurate, and thus introduces $3^{\text {rd }}$ order errors in the deformation calculation. Eq. 3.14 is expanded as,

$$
\frac{\rho t_{w}}{S_{\text {stress }}} \frac{a_{m n}^{t_{i}}-2 a_{m n}^{t_{i}-\Delta t}+a_{m n}^{t_{i}-2 \Delta t}}{\Delta t^{2}}+\pi^{2}\left(\frac{m^{2}}{c^{2}}+\frac{n^{2}}{b^{2}}\right) a_{m n}^{t_{i}-\Delta t}=-\frac{p_{m n}^{t_{i}-\Delta t}}{S_{\text {stress }}}(3
$$

The equation above is solved for $a_{m n}^{t_{i}}$, with known $a_{m n}^{t_{i}-\Delta t}$. An exception exists at $t_{i}=t_{2}$. At $t_{i}=t_{2}, a^{t_{i}-2 \Delta t}$ is unknown. The problem is resolved by knowing that $\dot{a}_{m n}^{t_{1}}=0$. By the application of central difference at time $t_{i}=t_{1}, a_{m n}^{t_{2}}=$ $a_{m n}^{t_{0}}=0$

- The structural coefficients can now be applied to Eq. 3.5 and the deformations at time $t=t_{i}$ can be computed. The velocity due to the deforming membrane, 
structural velocity $V_{s}$, is calculated using backward difference, and represented by,

$$
\dot{w}^{t_{i}}=\frac{w^{t_{i}}-w^{t_{i}-\Delta t}}{\Delta t}
$$

Using the backward differencing introduces errors of the second order in the velocity calculation.

- The structural velocity is computed in the wing frame, or the body axis. In order to compute the velocity in the inertial frame, $\dot{w}$ is multipled with the unit normal on the panel on which it is computed.

- The unit normal on the wing's plane changes because of the deformations experienced. Before the new normal vectors are calculated, the new vortex nodes have to be calculated after the deformation. This is done by the addition of $w(i, j) \vec{n}$ to the wing surface nodes. The normal can now be calculated by Eq. 2.40.

- The velocity acting on the control points is calculated for time $t=t_{i}$, and the no penetration condition is applied. 


\section{Chapter 4}

\section{Results}

\subsection{Validation of the 2-dimensional aspects of the Aerodynamic model}

The 3-dimensional VLM developed is validated with the 2-dimensional VLM for cases of constant angle of attack; pitch; plunge; and pitch and plunge. Certain parameters are kept constant for all the cases. These parameters and their values are, density of air $\rho=1 \mathrm{~kg} / \mathrm{m}^{3}$, freestream velocity $U_{\infty}=100 \mathrm{~m} / \mathrm{s}$, and wing chord $c=5 \mathrm{~m}$. Since the vortex strengths, and lift per unit span of the 3-dimensional VLM are compared with the corresponding values of a 2-dimensional VLM, it is imperative to have a very high aspect ratio. For this purpose, the span is assumed to be $9000 \mathrm{~m}$, giving 
the wing an aspect ratio of $A R=1800$. Having a high aspect ratio ensures that the 3-dimensional effects are negligible.

The 2-dimensional VLM code has been developed by the author, and the technique implemented can be found in Ref.[23]. The 2-dimensional code with free wake has been validated with a fixed wake model. Results from a 2-dimensional constant angle of attack case have been compared with the analytical results of Wagner, and results from cases of sinusoidal pitch and plunge motion have been compared to the analytical results of Theodorsen successfully [21].

\subsubsection{Constant Angle of Attack}

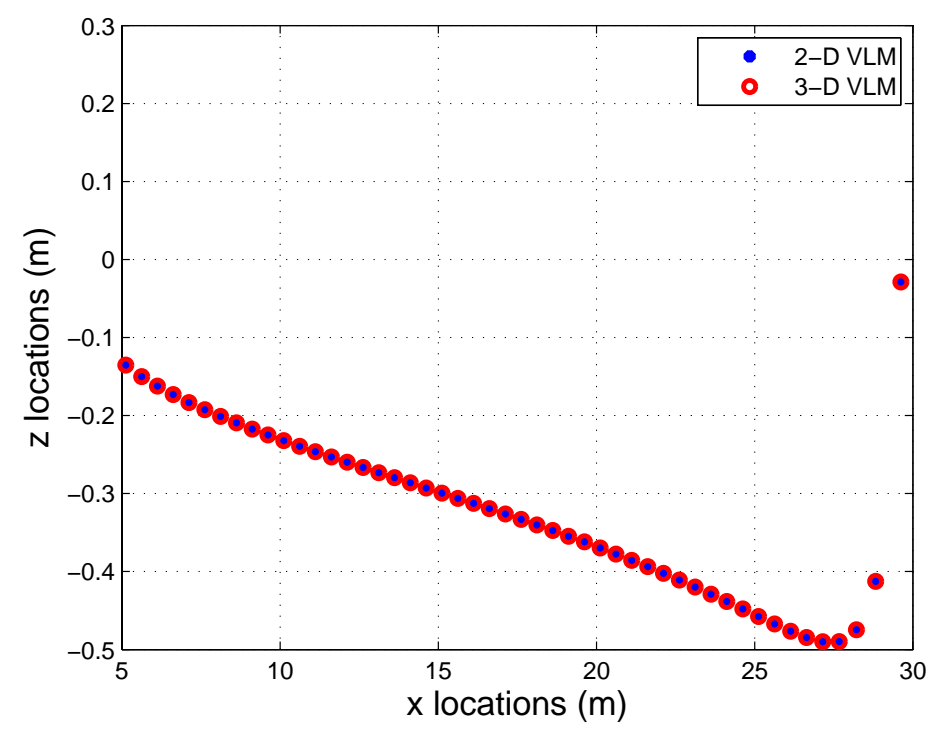

Figure 4.1: 2-D plot of the wake vortices after 50 time steps 


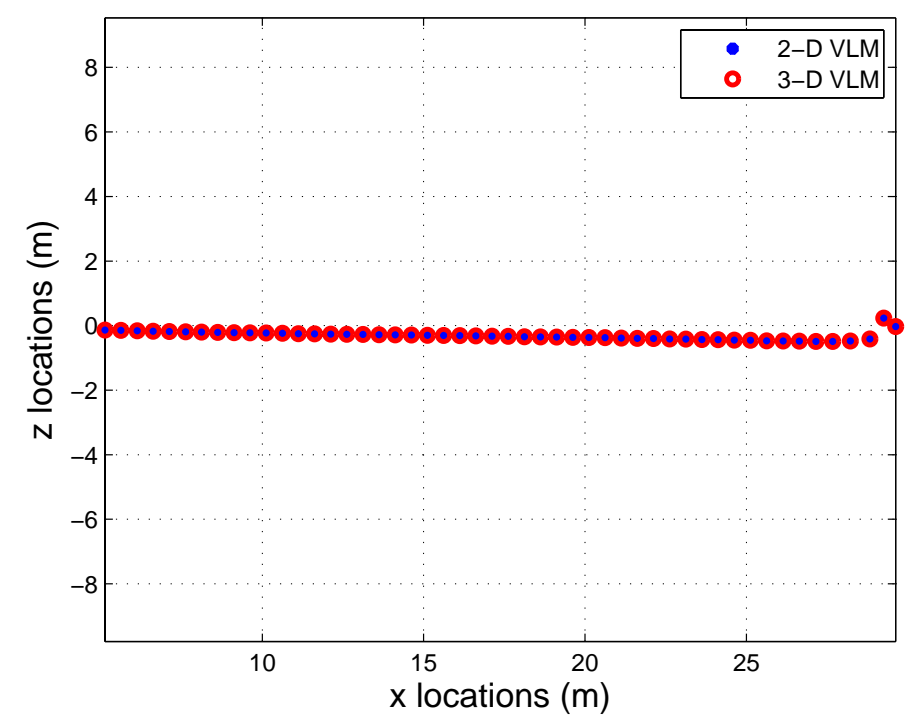

Figure 4.2: 2-D plot of the wake vortices after 50 time steps with equal axis

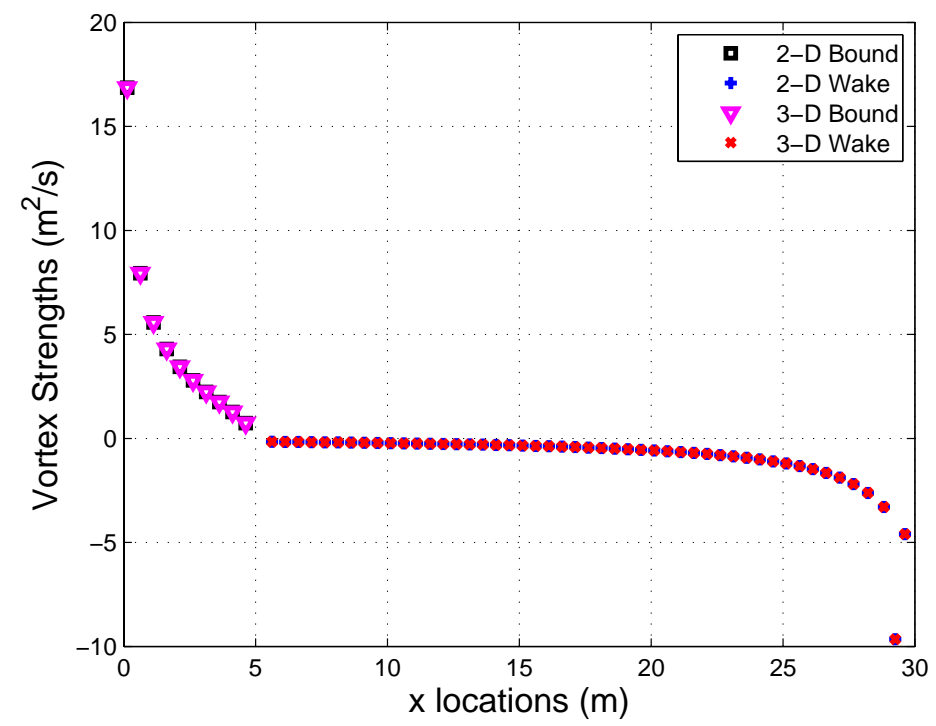

Figure 4.3: Comparison of Vortex strengths of 2-D and 3-D VLM after 50 time steps 


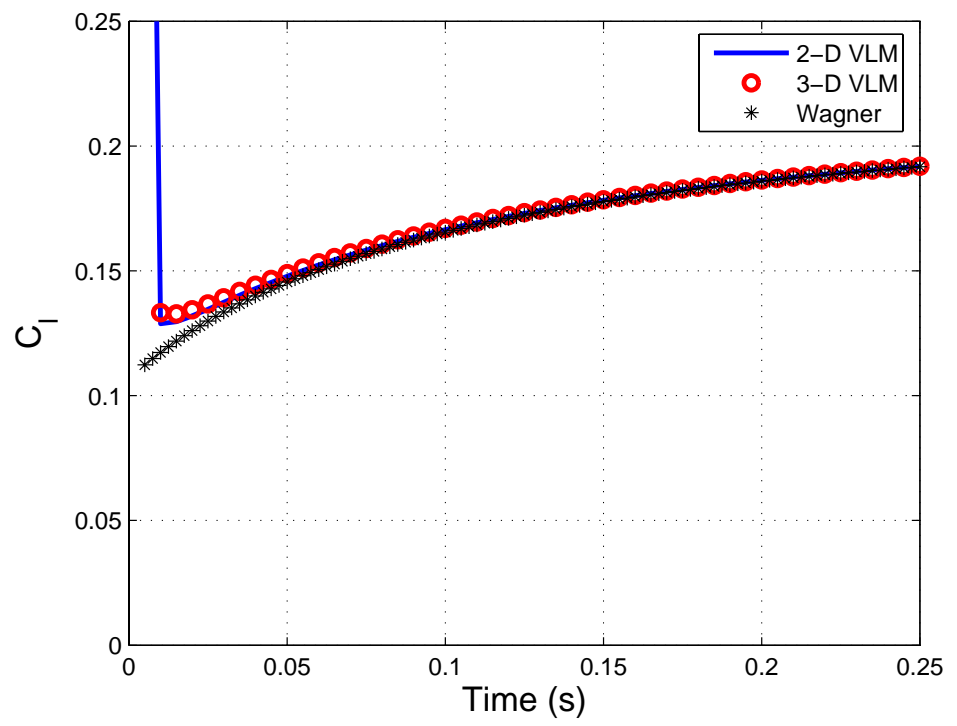

Figure 4.4: Comparison of 2-D Lift coefficient as a function of time

For an angle of attack of $2^{\circ}$, the plots of the locations of the wake vortices (2plots), the strengths of the bound and wake vortices, and the lift per unit span are given in Figures 4.1, 4.2, 4.3, and 4.4 respectively. The flow has been simulated for 50 time steps, which corresponds to 10 wing chord lengths, with each time step having a value of, $d t=0.005 \mathrm{~s}$.

In Figures 4.1 and 4.2, the wake locations of the vortices shed from the midspan of the wing at each of the time step are compared with the vortex locations of the vortices shed by the 2-dimensional VLM. The $\mathrm{x}$-location starts from 5 on the $x$ axis because the origin of the reference axis is the wing's leading edge and the wing has a $5 \mathrm{~m}$ chord length. The vortices are shed from the wing's trailing edge which is at 
$5 \mathrm{~m}$, and move in the positive $x$ direction.

The strengths of the vortex segments are compared in Figure 4.3. It can be seen that the values of the vortex strengths computed by the two methods are the same, thereby validating the 3-dimensional aerodynamic modeling for constant angle of attack. It has to be realized that the contribution of the free wake to the various parameters predicted is non-linear. The displacement of the vortices in the wake becomes insignificant in the calculation of the bound vortex strengths. The match in the results of the vortex locations and vortex strengths ensures that the wake effects have been effectively captured for constant angle of attack.

Figure 4.4 shows that the lift coefficient generated from the 2-dimensional VLM, and the 3-dimensional VLM match with the analytical results of Wagner [21]. The two VLM method's transition into the steady state is modeled for a sudden change in angle of attack from zero degrees. The lift calculation in the VLM is a sum of a steady and unsteady lifts. At the initial time steps, the unsteady loads are high due to vortices of higher strengths being shed into the wake. As time passes by, the unsteady part becomes less dominant, and the lift results converge to the steady state lift. 


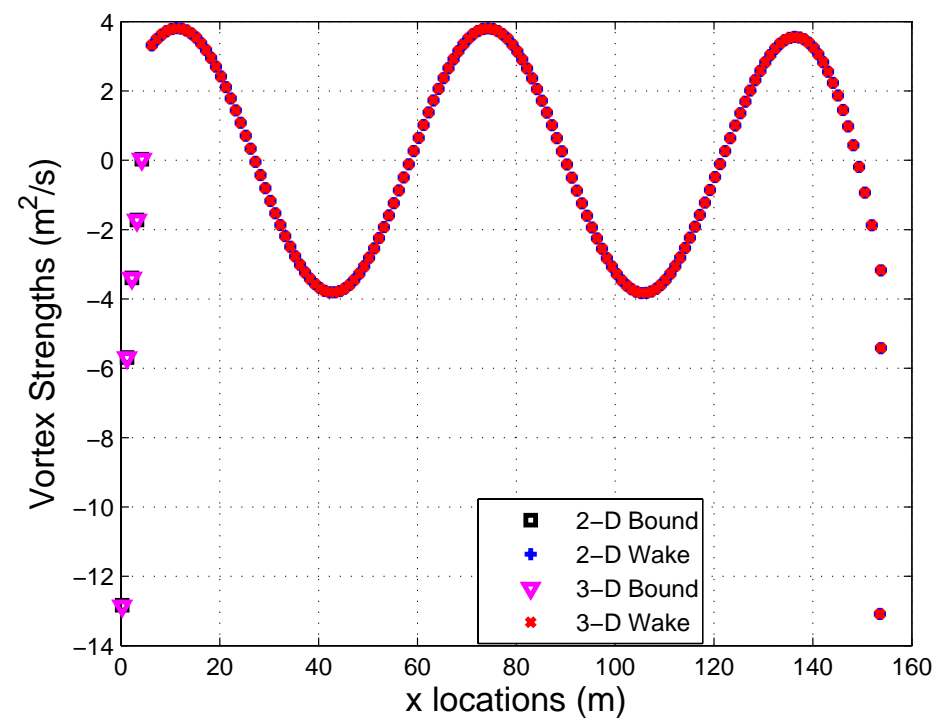

Figure 4.5: Comparison of Vortex Strengths of 2-D and 3-D VLM

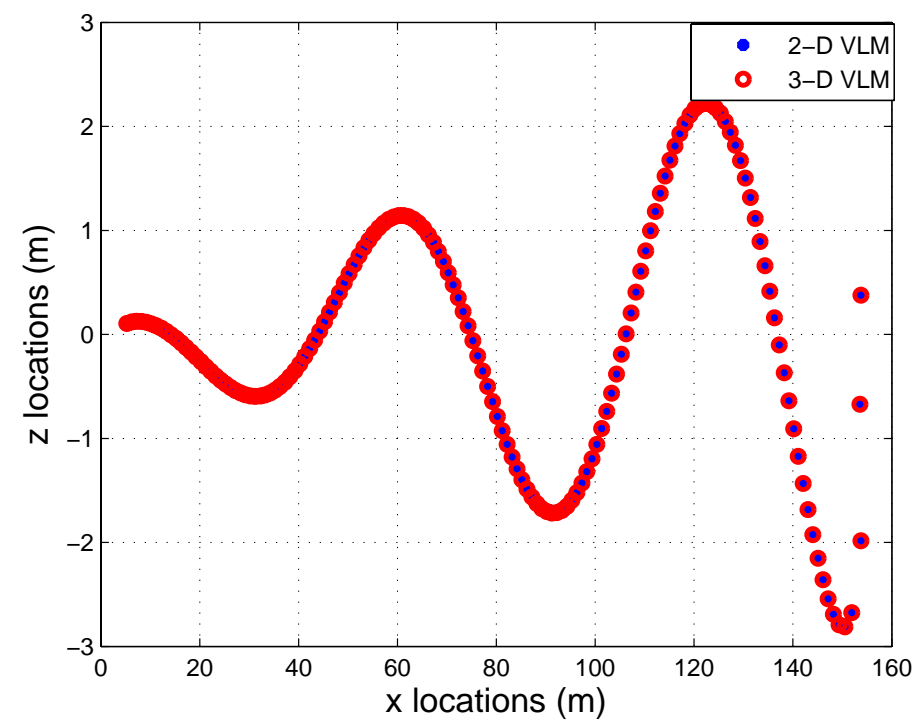

Figure 4.6: 2-D plot of the location of the wake vortices after 150 time steps 


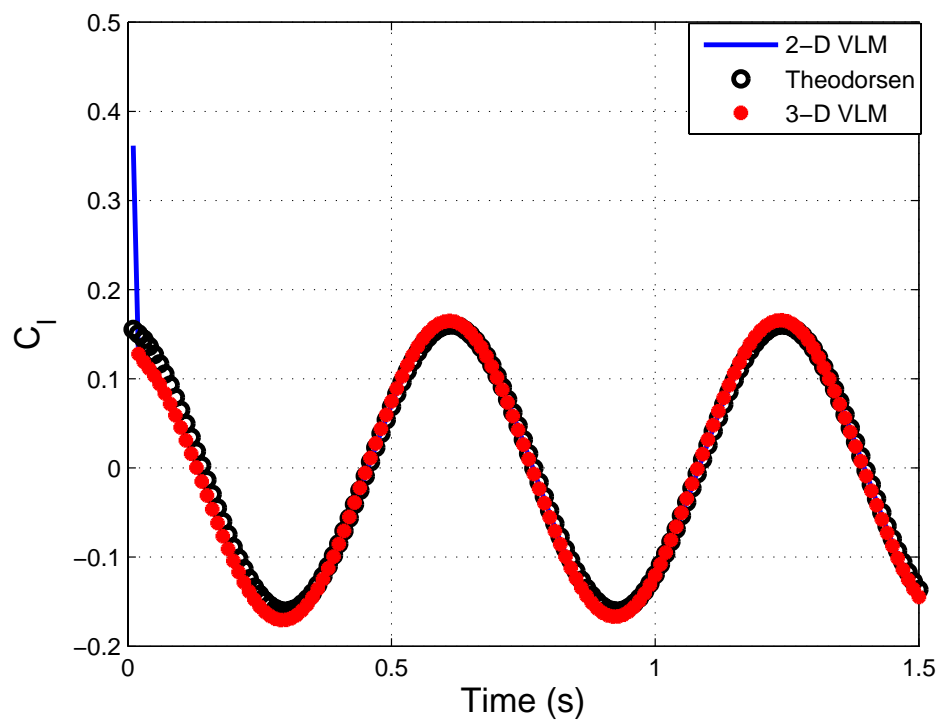

Figure 4.7: 2-D lift coefficient developed by 2-D and 3-D VLM as a function of time

\subsubsection{Pitching wing}

For the case of pitch, the parameters and their values are as follows; angle of attack amplitude $\alpha_{0}=2^{o}$; reduced frequency $k=0.25$; number of time steps of $n=150$; and time interval $d t=0.01 \mathrm{~s}$. Also, for this case the number of vortex panels in the chordwise and spanwise directions are 5 and 20 respectively. As can be seen from Figures $4.5,4.6$, and 4.7 the vortex strengths, wake locations and lift per unit span generated by the 3-dimensional VLM are in agreement with the results generated by the 2-dimensional method. The lift per unit span is also in agreement with the analytical results of Theodorsen [21]. 


\subsubsection{Plunging wing}

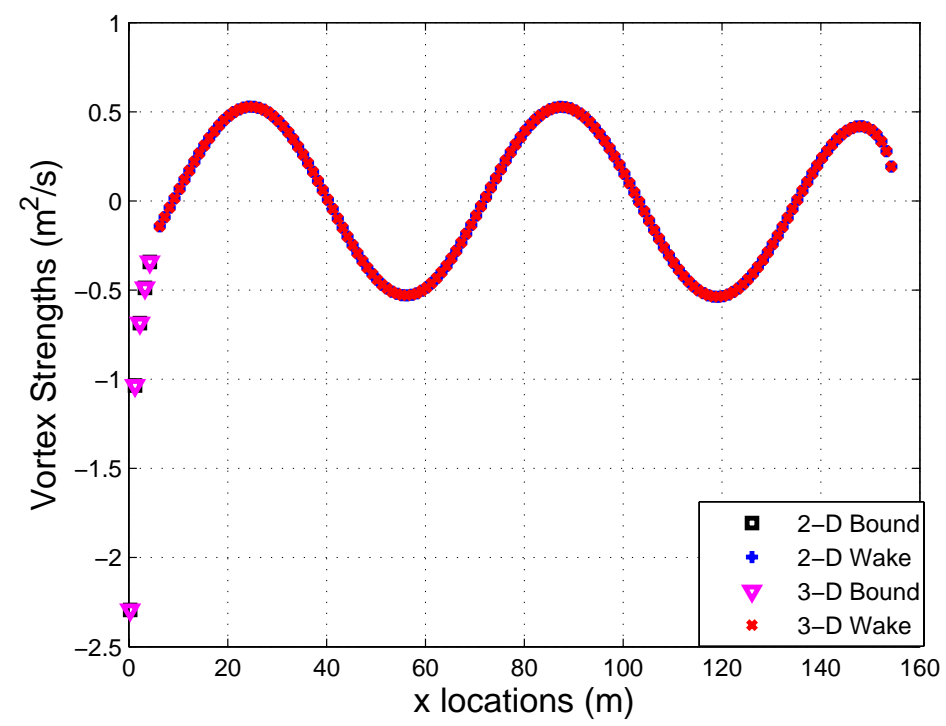

Figure 4.8: Comparison of Circulation of 2-D and 3-D VLM

For the case of plunge, the parameters and their values are as follows; plunge amplitude of $h_{0}=0.01 \mathrm{~m}$; reduced frequency $k=0.25$; number of time steps of $n=150$; number of rows of vortex ring elements, 5 ; number of columns of vortex ring elements, 20; and time interval $d t=0.01 \mathrm{~s}$. As can be seen from Figures 4.8, 4.9, and 4.10 the vortex strengths, wake locations and lift per unit span generated by the 3-dimensional VLM are in agreement with the results generated by the 2dimensional method. The lift per unit span is also in agreement with the analytical result of Theodorsen. 


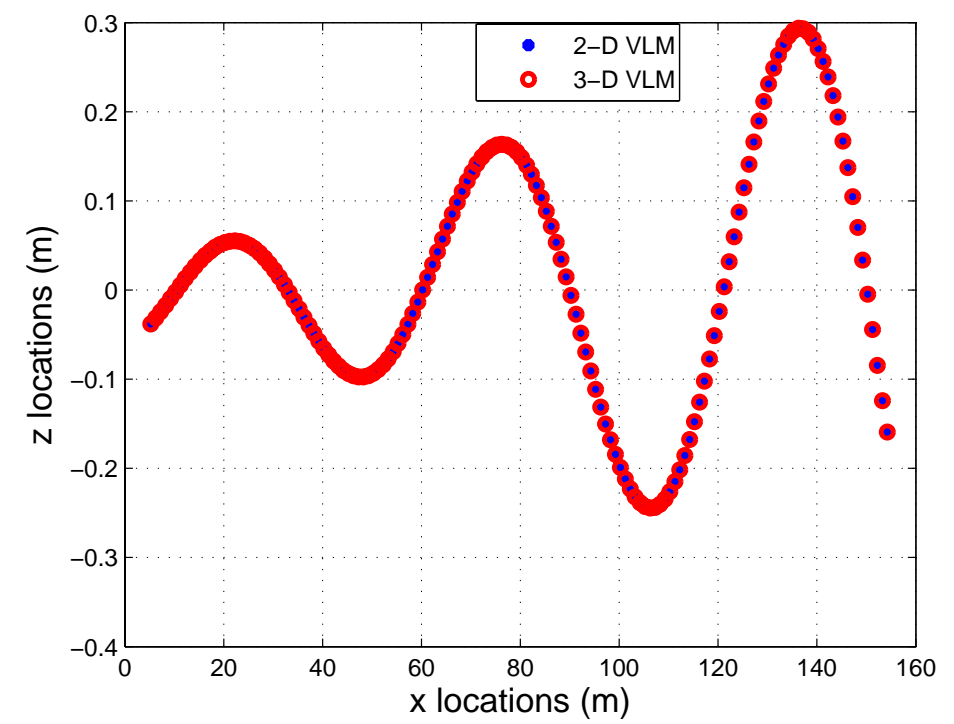

Figure 4.9: 2-D plot of the location of the wake vortices after 150 time steps

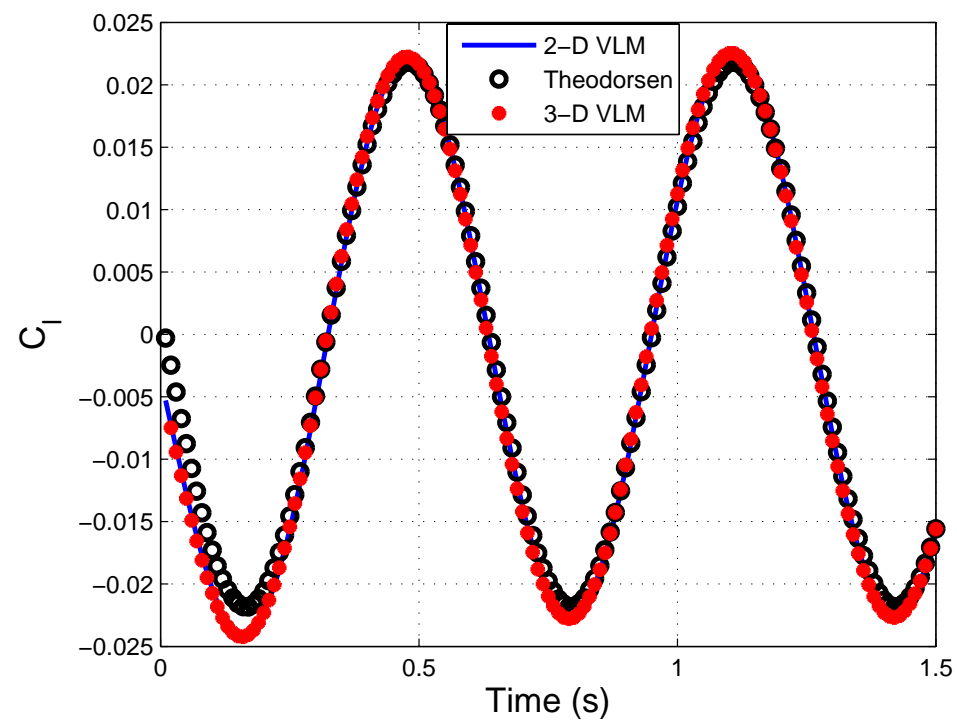

Figure 4.10: 2-D lift coefficient developed by 2-D and 3-D VLM 


\subsection{Grid discretization studies}

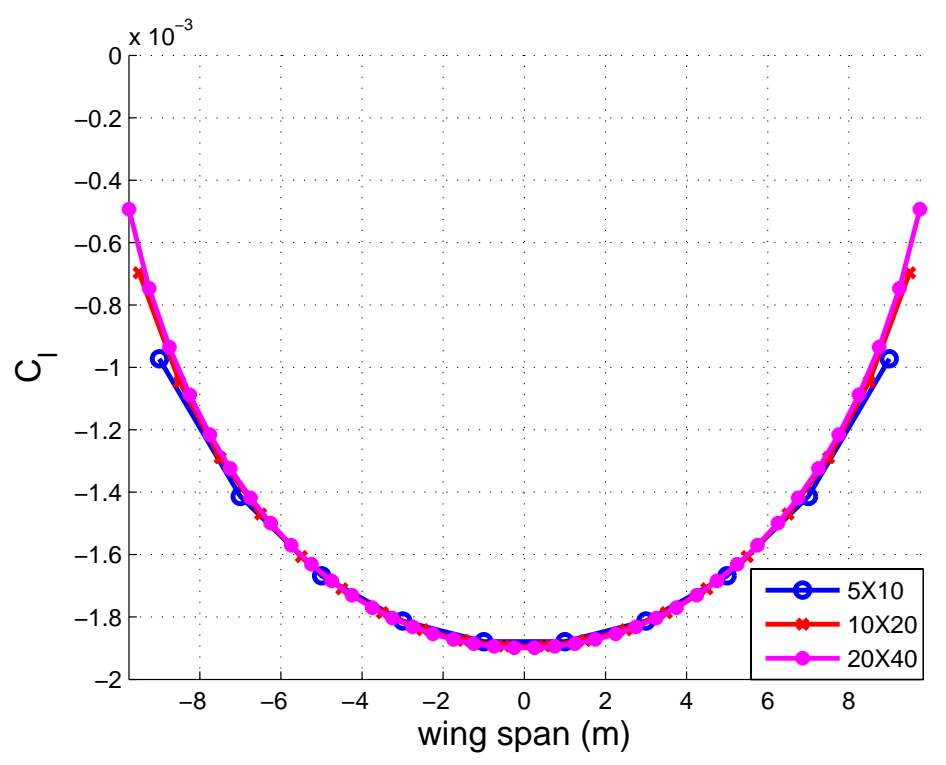

Figure 4.11: $C_{l}$ from grid discretization study for wing in oscillating plunge

In order to make sure that the results generated are converged, the spanwise lift and the circulation are compared for three different grid configurations. The results generated are for a wing of span $20 \mathrm{~m}$, and a chord length of $5 \mathrm{~m}$. The freestream velocity is $100 \mathrm{~m} / \mathrm{s}$. For the case of plunge, the parameters used are; plunge amplitude of $h_{0}=0.01 \mathrm{~m}$, and reduced frequency $k=0.25$. For the case of pitch, the parameters and their values are as follows; angle of attack amplitude $\alpha=5^{\circ}$, and reduced frequency $k=0.25$. The results are compared for grid sizes of 5 chordwise and 10 spanwise panels; 10 chordwise and 20 spanwise panels; and 20 chordwise and 40 spanwise panels. The results are plotted in Figures 4.11 and 4.12 for the plunging 


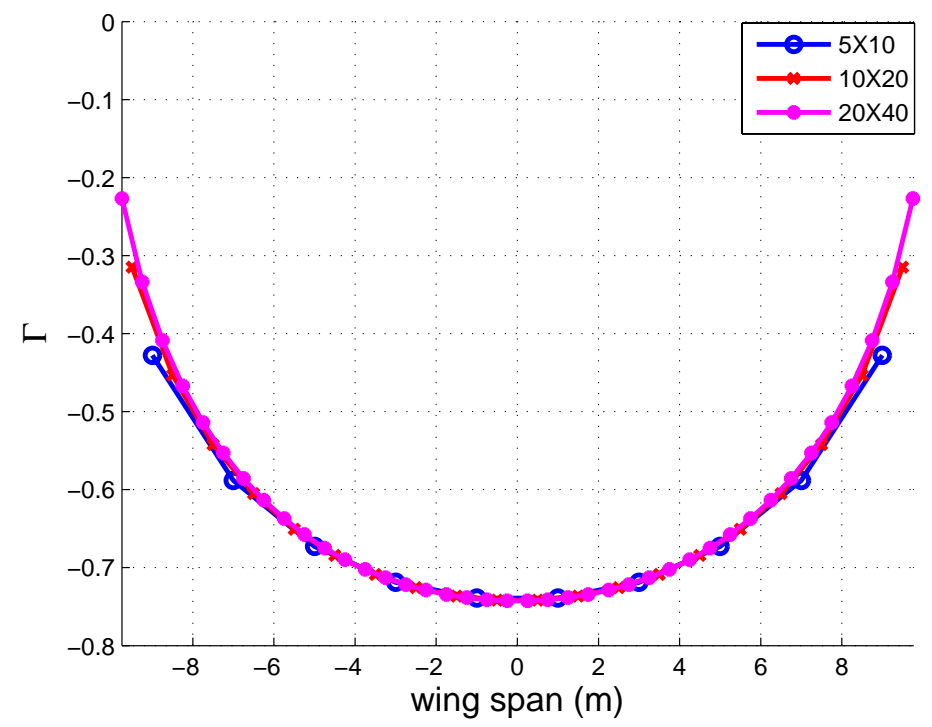

Figure 4.12: $\Gamma$ from grid discretization study for wing in oscillating plunge

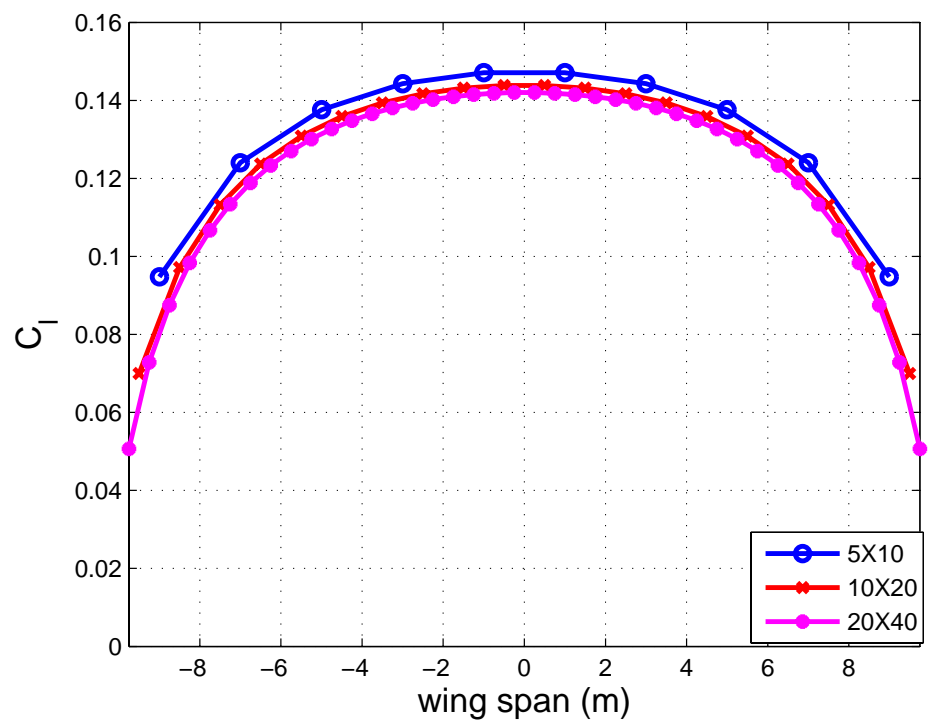

Figure 4.13: $C_{l}$ from grid discretization study for wing in oscillating pitch 


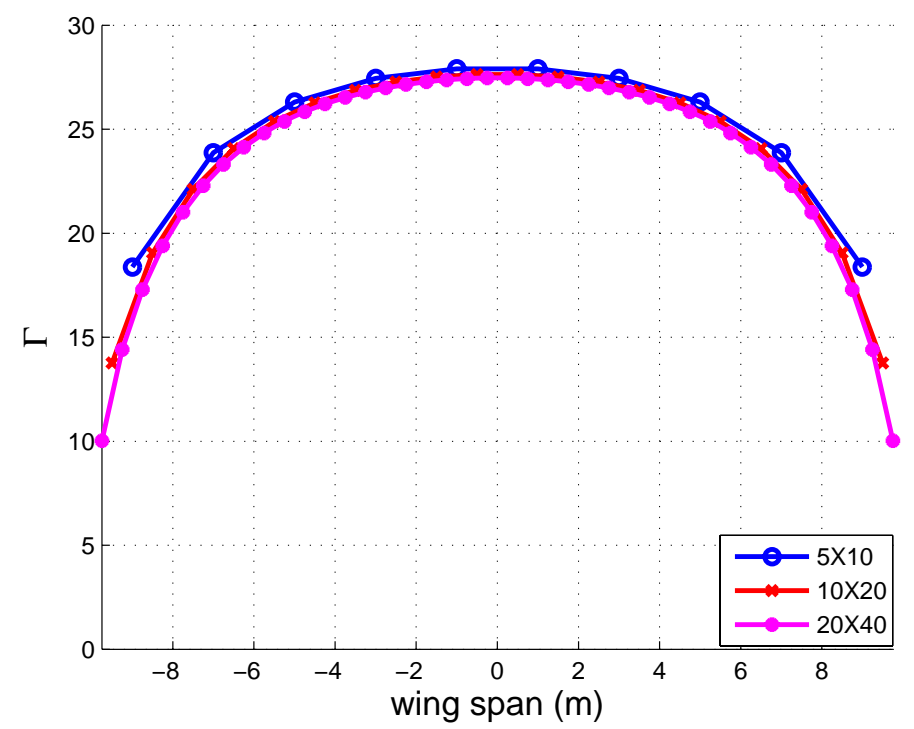

Figure 4.14: $\Gamma$ from grid discretization study for wing in oscillating pitch

case, and in Figures 4.13 and 4.14 for the pitching case.

It can be seen that the results for the three different grid discretization are converged. It can therefore be concluded that the results presented have insignificant grid discretization related errors.

\subsection{Comparison with lifting line theory}

The 3-dimensional VLM is compared with the lifting line theory for constant angle of attack. The lifting line theory provides lift and drag for a 3-dimensional wing using discrete horseshoe vortices [24]. For constant angle of attack of 5 degrees, wing span of $20 \mathrm{~m}$, chord length of $5 \mathrm{~m}$, freestream velocity of $100 \mathrm{~m} / \mathrm{s}$, and density of 1 


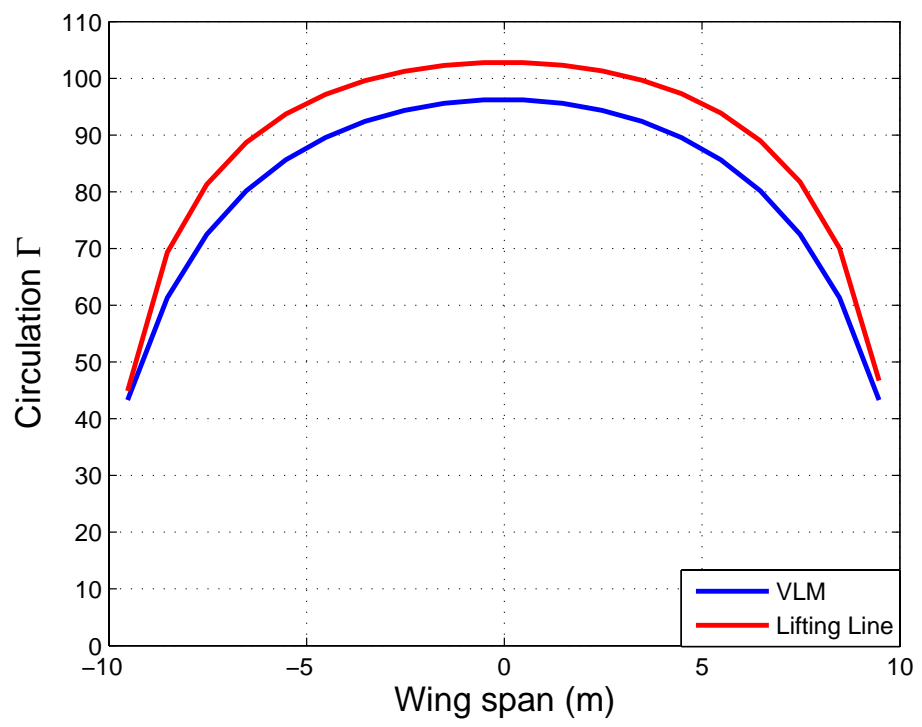

Figure 4.15: Plot of the spanwise circulation for $\alpha=5^{0}$

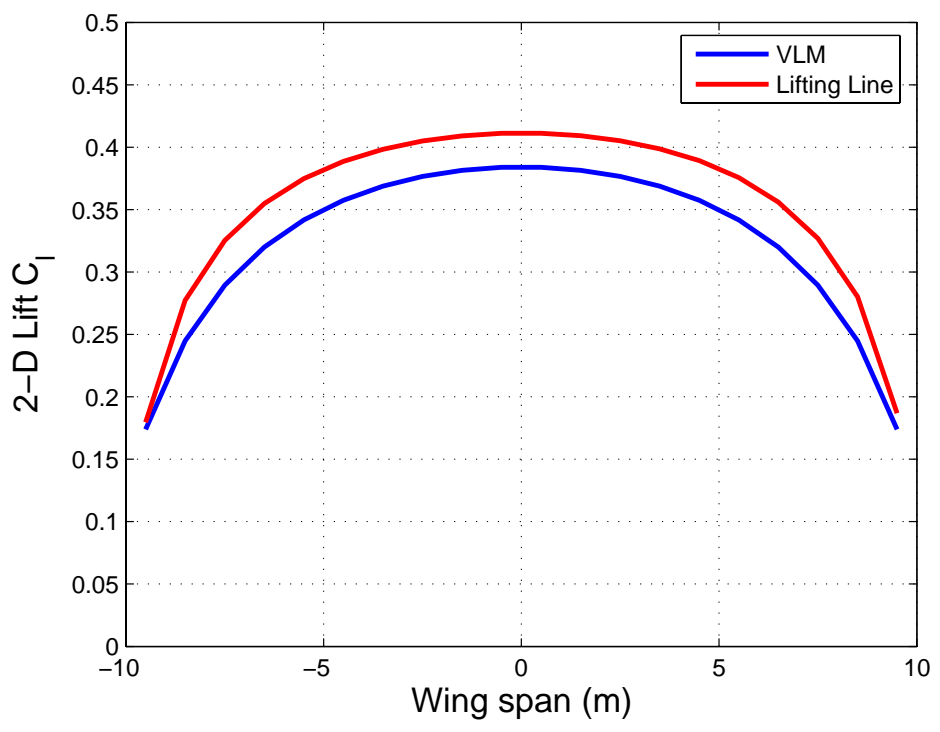

Figure 4.16: Plot of 2-D $C_{l}$ for $\alpha=5^{0}$ 
$\mathrm{kg} / \mathrm{m}^{3}$, the spanwise circulation is compared in Figures 4.15 and 4.16 . It can be seen that the circulation and 2-dimensional lift coefficient estimated by the 3-dimensional method are very close to those predicted by the lifting line.

\subsection{Validation of 3-dimensional aspects of VLM with Doublet Lattice method}

The 3-dimensional aspect of the aerodynamic modeling is validated with the doublet lattice method which can be found in Ref.[25]. The 3-dimensional VLM and DLM with 10 chordwise panels and 20 spanwise panels are considered for a wing of span $20 \mathrm{~m}$, and with a chord length of $5 \mathrm{~m}$. The code is validated for cases of pitch and plunge. The freestream velocity is $100 \mathrm{~m} / \mathrm{s}$, reduced frequency is 0.25 , and $d t$ is 0.005 S.

\subsubsection{Pitch}

For a pitch amplitude of $\alpha_{0}=2^{0}$, the amplitude of lift coefficient per unit span is compared in Figure 4.17. The VLM is run for 400 time steps, and the $C_{l}$ amplitude is determined from the last period of data. The phase angles for the results over the span of the wing is shown in Figure 4.18. The closeness in the results validates the 


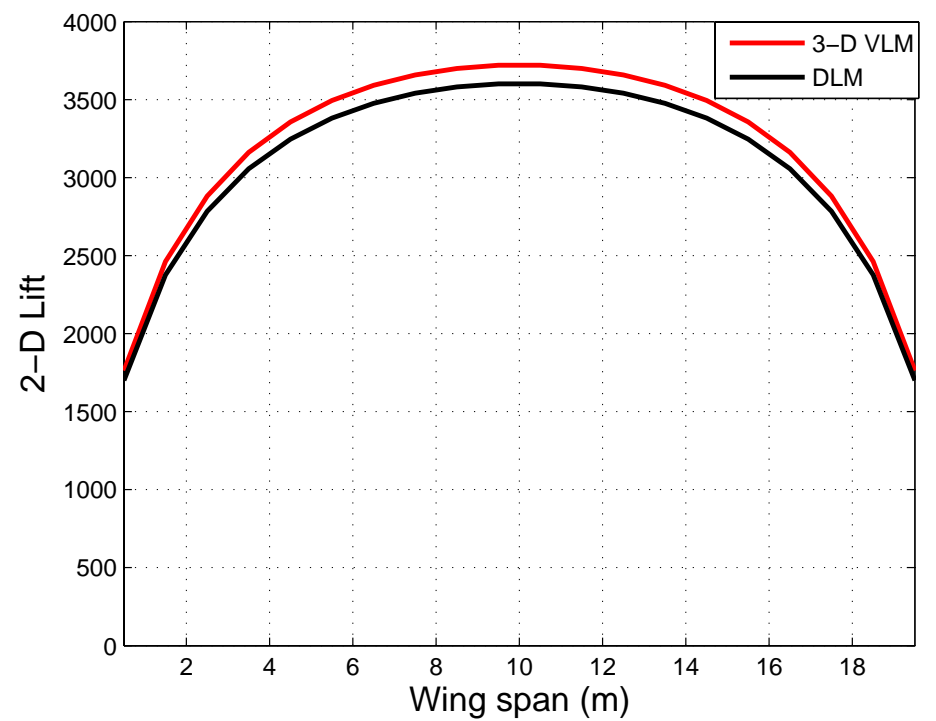

Figure 4.17: Comparison of $C_{l}$ from 3-D VLM and DLM for pitch

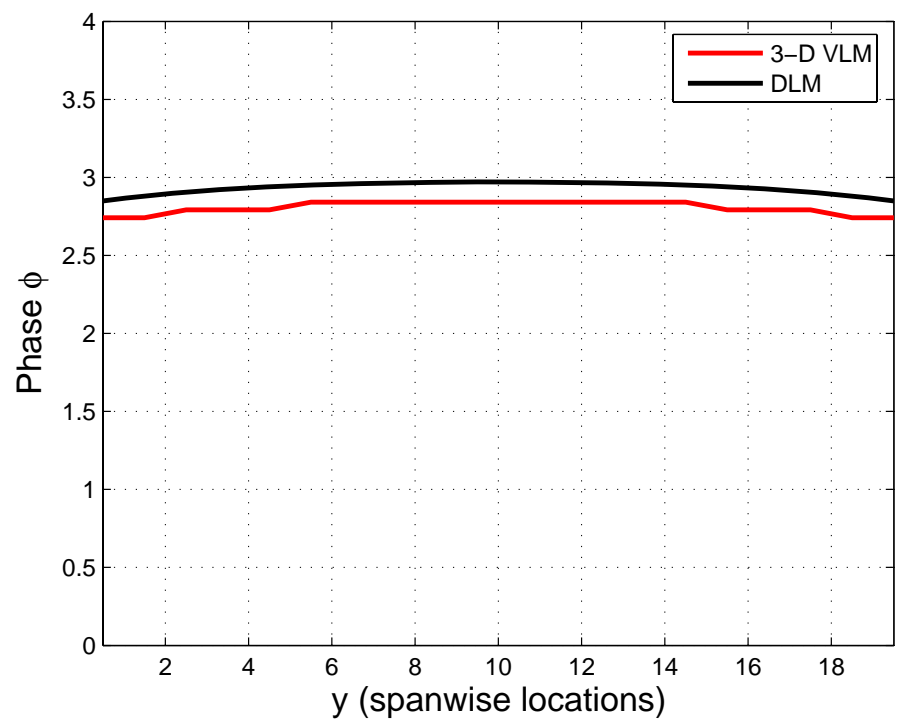

Figure 4.18: Comparison of the phase angle between the input pitch and the $C_{l}$ 
3-dimensional VLM.

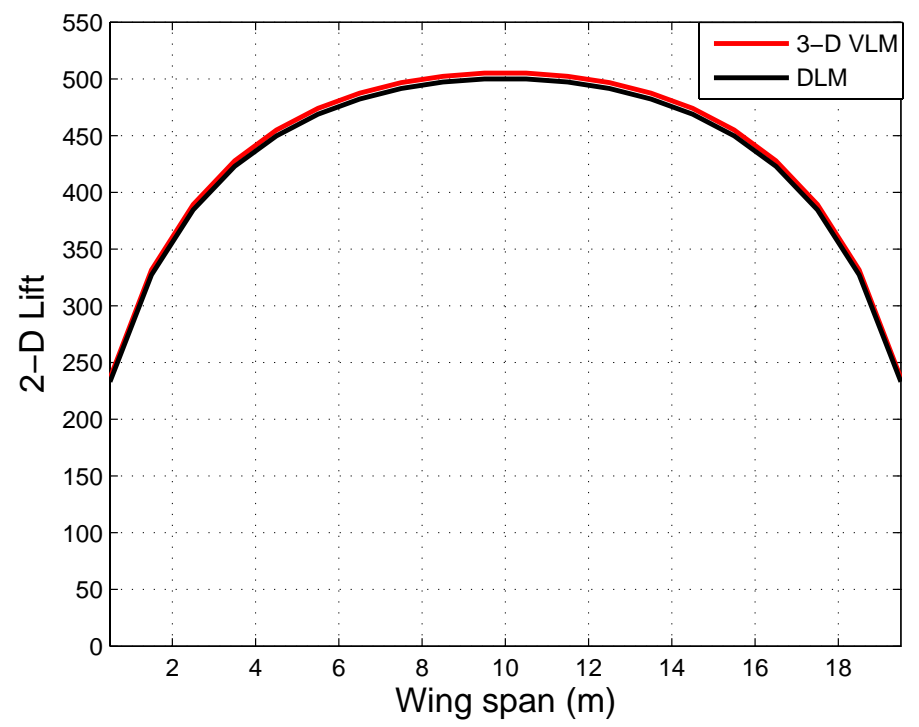

Figure 4.19: Comparison of $C_{l}$ from 3-D VLM and DLM for plunge

\subsubsection{Plunge}

For a plunge amplitude of $h_{0}=0.01 \mathrm{~m}$, the amplitude of lift coefficient per unit span is compared in Figure 4.19. Again, the VLM is run for 400 time steps, and the $C_{l}$ amplitude is determined from the last period of data. The phase angles for the results over the span of the wing is shown in Figure 4.20. 


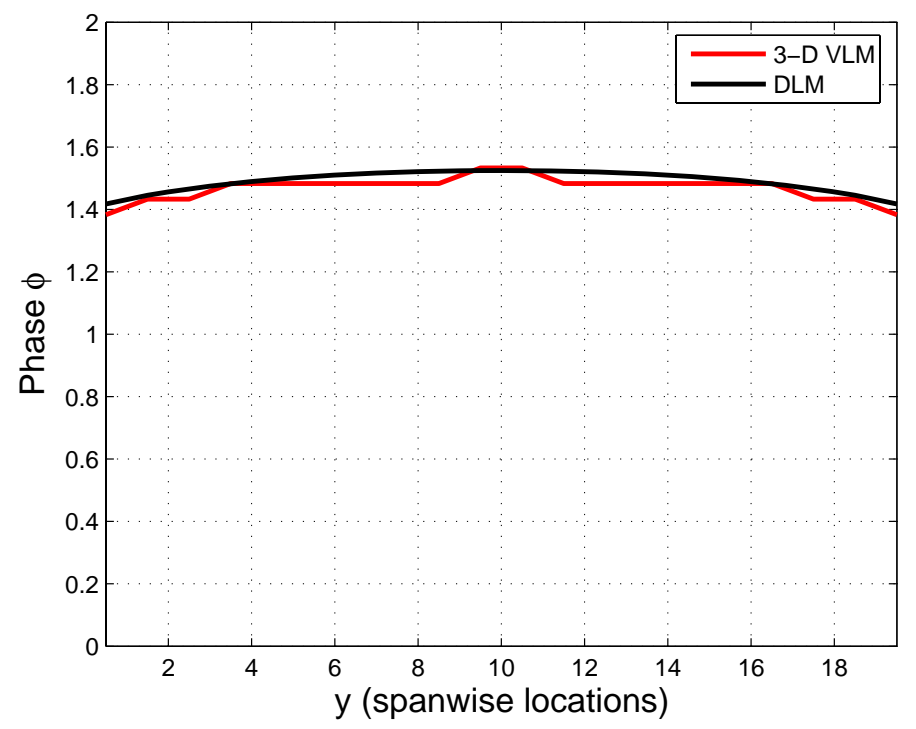

Figure 4.20: Comparison of the phase angle between the plunge and the $\mathrm{Cl}$

\subsection{Study of loads and deformations for constant angle of attack}

In this section of the thesis, the rectangular wing, with chord length and span of 0.1 $\mathrm{m}$ and $0.5 \mathrm{~m}$ respectively, is studied for a constant angle of attack of $5^{\circ}$. The wing has a rigid boundary, and it supports a thin membranous surface. The membrane is modeled with the properties of nylon, material density of $\rho_{m}=1200 \mathrm{~kg} / \mathrm{m}^{3}$, and has a thickness of $t_{w}=0.00025 \mathrm{~s}$. The membrane is modeled with a pre-stress of $S_{\text {stress }}=10 \mathrm{~N} / \mathrm{m}$ and $m=3, n=3$ number of structural modes. The membrane wing is allowed to deform under the influence of the loads. The freestream velocity 
and density are $U_{\infty}=10 \mathrm{~m} / \mathrm{s}$ and $\rho=1 \mathrm{~kg} / \mathrm{m}^{3}$ respectively; there are 10 rows and 20 columns of vortex panels on the surface of the wing, and the study is performed for 400 time steps. Each time step has a value of $d t=0.001 \mathrm{~s}$. The case for the properties mentioned above is referred to as the base case, and the various parametric studies conducted are compared and analyzed with respect to the base case. The drag results do not take the leading edge drag into account. These studies are described below.

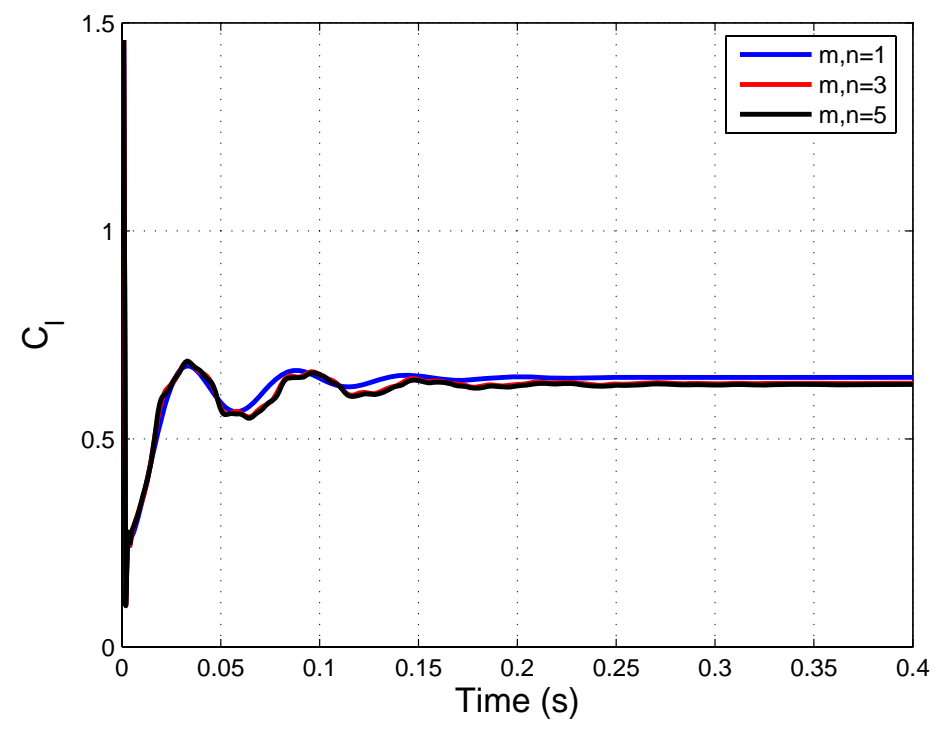

Figure 4.21: Plot of $C_{l}$ versus time for constant angle of attack of $5^{\circ}$ and $\mathrm{S}=10$ 


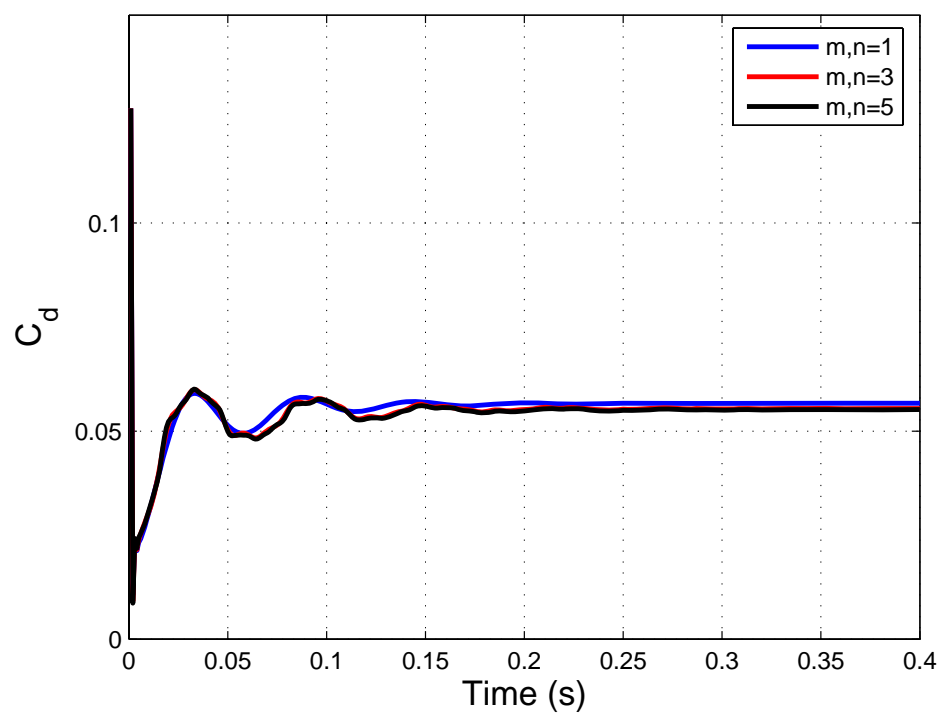

Figure 4.22: Plot of $C_{d}$ versus time for angle of attack of $5^{\circ}$ and $\mathrm{S}=10$

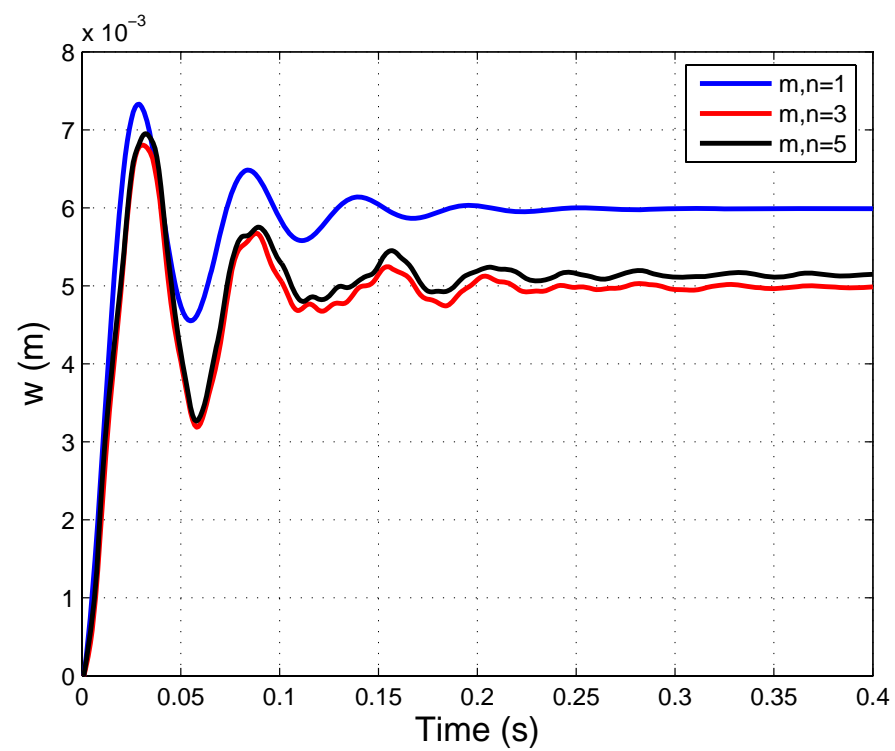

Figure 4.23: Plot of $w$ versus time at approximately the center of the wing for angle of attack of $5^{\circ}$ and $S=10$ 


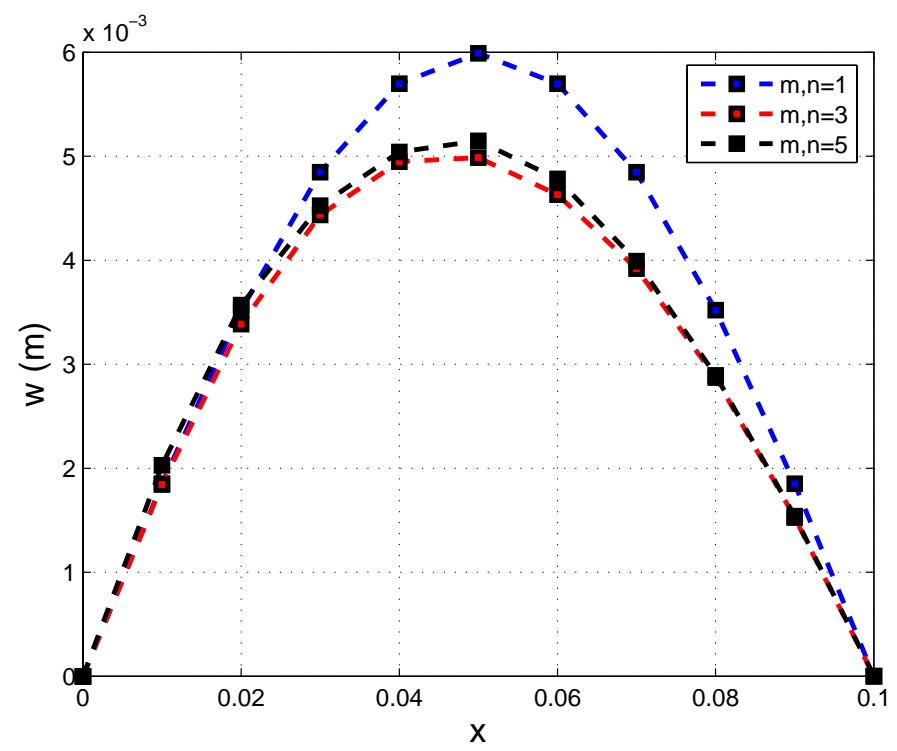

Figure 4.24: Plot of the deformation at the midspan of the wing for an angle of attack of $5^{\circ}$ and $\mathrm{S}=10$

\subsubsection{Variation in the number of structural modes}

The number of modes used to define the structural deformation is varied from values

of $m=n=1$ to $m=n=5$. The pre-stress stiffness is assigned a value of $S_{\text {stress }}=10$

$\mathrm{N} / \mathrm{m}$. Other parameters are kept constant and the influence on the lift coefficient, drag coefficient, the deformation of the wing's center, and the deformation at the wing's midspan are analyzed. For all three cases, the lift and the drag coefficients oscillate and damp out in 250 time steps. The case where $m=n=1$ oscillates with one frequency as there is only one mode defining the deformation; whereas the cases with multiple modes oscillate with more than one frequency as seen in Figure 4.23. 
The wing is set into motion from rest at the initial time step. The membrane is at an undeformed state at this time. The step change in angle of attack causes a step change in the aerodynamic load, and a corresponding high lift. The aerodynamic loads causes it to oscillate about the steady state deformation. Some of the oscillation may be caused by the initial load as well.

The deformation leads to a relative angle of attack for any point on the membrane. Thus, the total angle of attack at any point on the membrane is the sum of the angle of attack of the rigid wing and relative angle of attack due to the membrane deformation. Since, the pressure acts normal to the surface of the membrane, these deformations bring about a change in the aerodynamic loads by changing the unit normal vector. Figure 4.24 shows the deformation at the midspan on the wing's surface. It can be seen that the deformation calculated using one structural mode is symmetric about the midchord, whereas the deformation calculated using multiple structural modes has the highest deformation slightly toward the leading-edge side of the midchord and is lower in value.

The symmetry in the deformation curve for the $m=n=1$ case is expected, as only one sinusoidal function with zero deformation at the boundary is used to predict the deformation. For this case, the region ahead of the point experiencing the maximum deformation has a lower angle of attack, and the region aft of this 
point has higher angle of attacks. When more modes are used in the prediction of the deformation the amplitude and shape of the deformation calculated is more accurate.

The shift in the highest deformation on the midspan toward the leading edge of the wing can be attributed to higher pressure around the leading edge of the wing. As compared to the $m=n=1$ case, the slope of the deformation at the leading edge is lower, and therefore the total angle of attack experienced by the membrane in this region is higher than the $m=n=1$ case. The slope of the deformation keeps on decreasing as the deformation increases and reaches its peak. Beyond its peak, the slope of the deformation is negative just as the $m=n=1$ case. However, its magnitude is lower than the $m=n=1$ case. The lower magnitude results in the region beyond the peak of the deformation curve experiencing a lower angle of attack than the $m=n=1$ case. Since the deformation calculated for the $m=n=3$ case is lower, the loads calculated are lower too than the $m=n=1$ case.

The deformation of the membrane at $m=n=3$ and $m=n=5$ is lower as compared to deformation of the membrane for $m=n=1$, as seen in Figure 4.24. This is expected because for the cases of $m=n=3$ and $m=n=5$ the deformed shape is such that the reduction in angle of attack due to membrane deformation is magnified and the increase in the angle of attack at the trailing edge due to membrane 
deformation is attenuated. The overall effect of the higher accuracy at $m=n=3$ is that the deformation leads to a decrease in the increase of angle of attack which leads to the decrease in the aeroelastic loads and thus lower deformation.

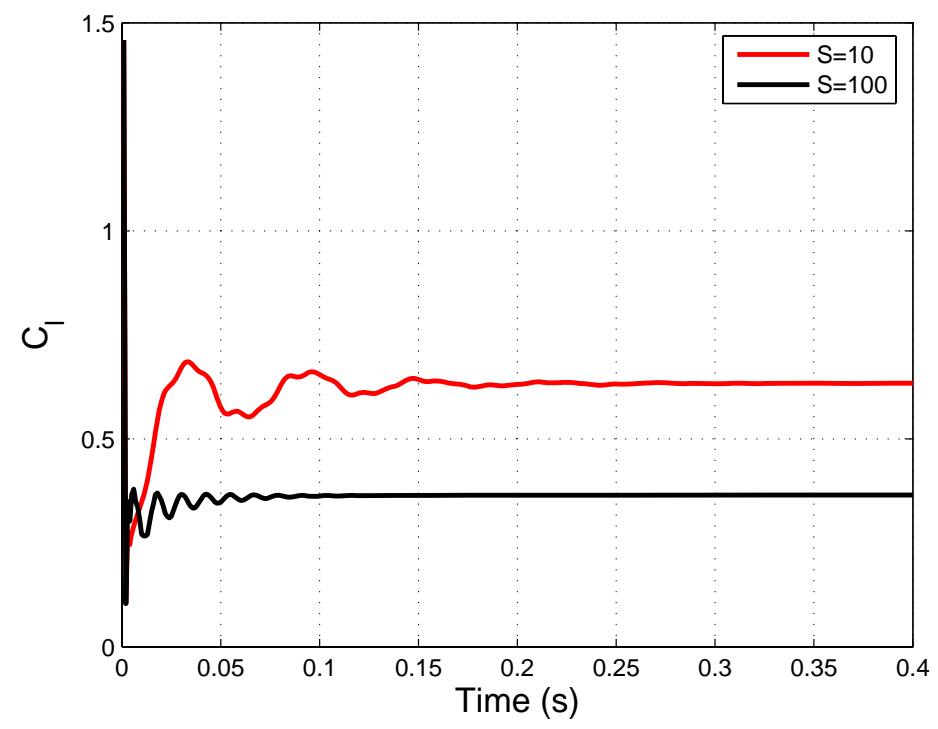

Figure 4.25: Plot of $C_{l}$ versus time for constant angle of attack of $5^{\circ}$

\subsubsection{Variation in stiffness}

The base case is compared to a case where the pre-stress stiffness is increased, and its influence on the loads and deformations are analyzed. The base case is compared to a case with a higher value of pre-stress, $S_{\text {stress }}=100 \mathrm{~N} / \mathrm{m}$. The higher stiffness provides higher resistance against deformation, and is evident in Figure 4.27. The deformation for the higher pre-stress is $1 / 20^{\text {th }}$ the deformation of the base case. As 


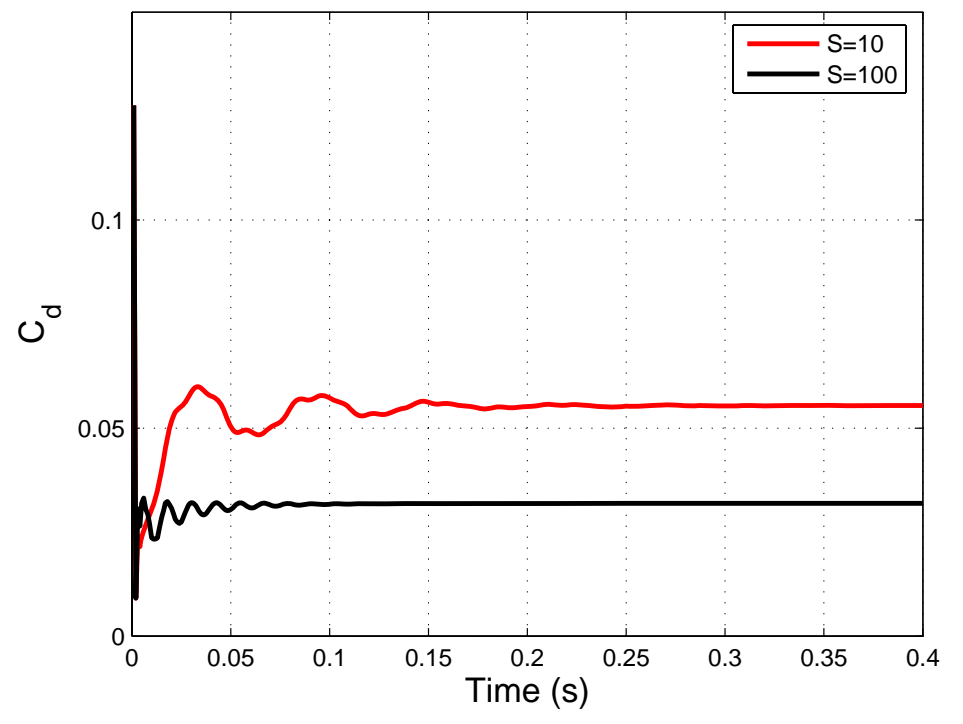

Figure 4.26: Plot of $C_{d}$ versus time for angle of attack of $5^{\circ}$

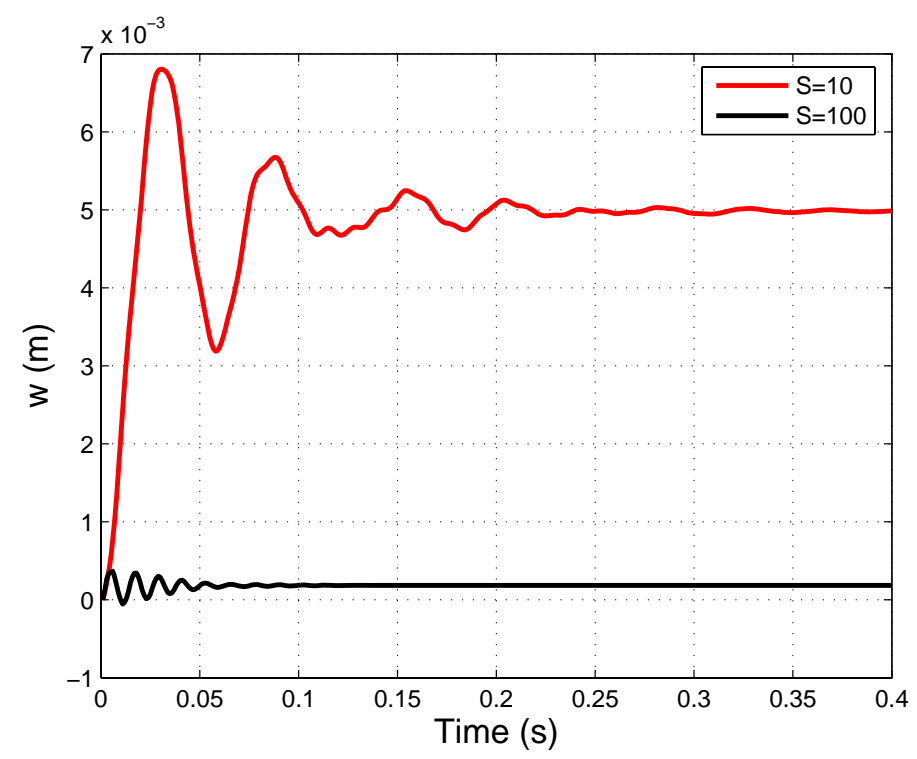

Figure 4.27: Plot of $w$ versus time at approximately the center of the wing for angle of attack of $5^{\circ}$ 


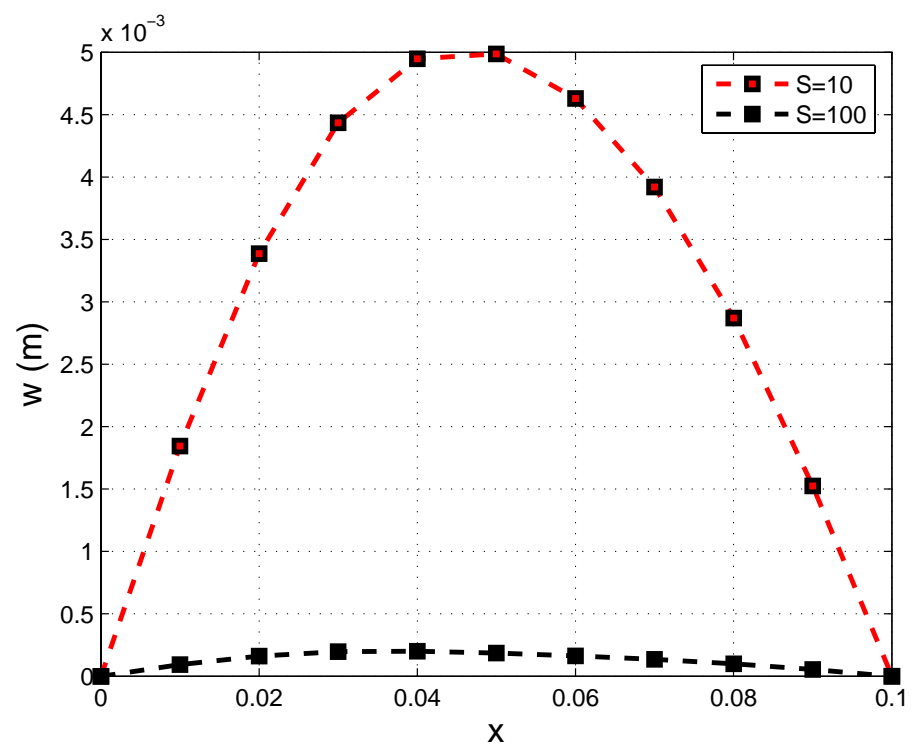

Figure 4.28: Plot of the deformation at the midspan of the wing for an angle of attack of $5^{\circ}$

the stiffness is increased 10 times, the deformation will decrease by 10 times just based on the loads at $5^{\circ}$ angle of attack. This lower deformation also leads to lower aeroelastic loads, and thus in Figure 4.25 the $C_{l}$ for the higher pre-stress is onehalf the $C_{l}$ of the base case. The combination of lower aeroelastic loads and higher stiffness leads to much smaller deformation for the stiffer case(1/20th).

Also, the frequency of oscillation in the $C_{l}, C_{d}$ and the wing center's deformation values increases for higher pre-stress, and the damping occurs much earlier, see Figures $4.27,4.25$ and 4.26 . The effect of higher modes in the response for the higher pre-stress case appears to be negligible, and the frequency of oscillation for the load and the deformations being analyzed appear to be oscillating at one frequency. 
As seen in Figure 4.28, after 400 time steps, the deformation along the midspan of the wing is much lower for the higher pre-stress case as compared to the base. Also, the peak of the deformation occurs much ahead of the midchord for the higher pre-stress case.

\subsection{Study of loads and deformations for a plung- ing wing}

In this section, a rectangular wing undergoing plunge is studied and analyzed. The freestream velocity and density for the base case are $U_{\infty}=10 \mathrm{~m} / \mathrm{s}$ and $\rho=1$ $\mathrm{kg} / \mathrm{m}^{3}$ respectively. The pre-stress is $S=100 \mathrm{~N} / \mathrm{m}$, number of structural modes $m=n=3$, reduced frequency of 0.25 and has a plunge amplitude of $h_{0}=0.01 \mathrm{~m}$. Various parametric studies are performed and compared with the base case. The drag results do not take the leading edge drag into account. The studies and their results are described below.

\subsubsection{Variation in the number of structural modes}

The base case, with $m=n=3$ number of structural modes that are used in the calculation of deformation, is compared with a case having $m=n=1$ structural 


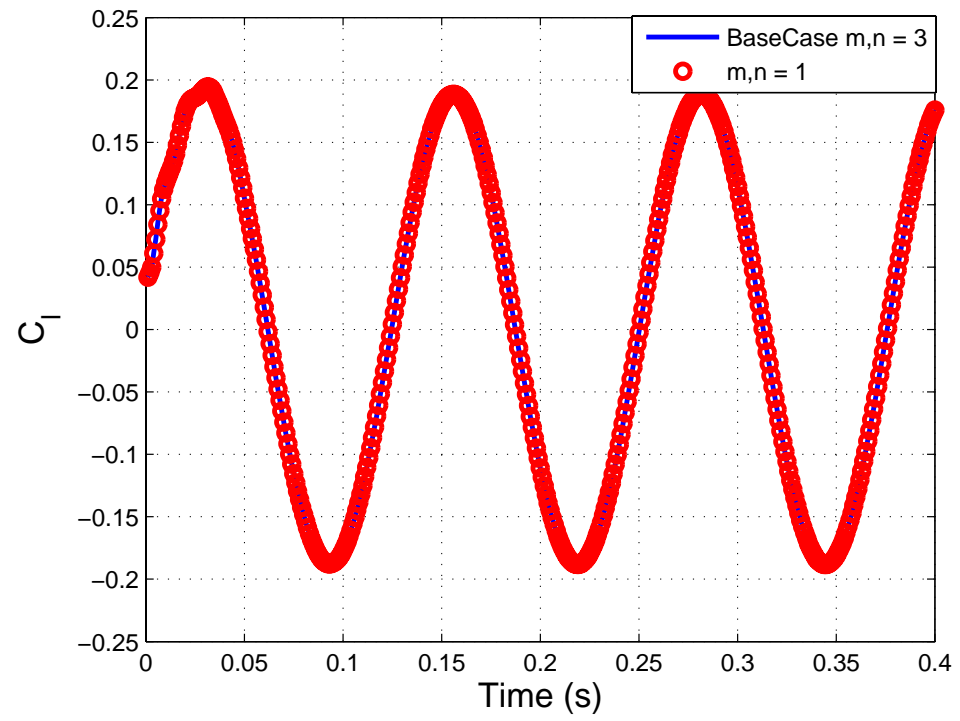

Figure 4.29: Plot of $C_{l}$ Vs Time for different number of structural modes for a plunging wing

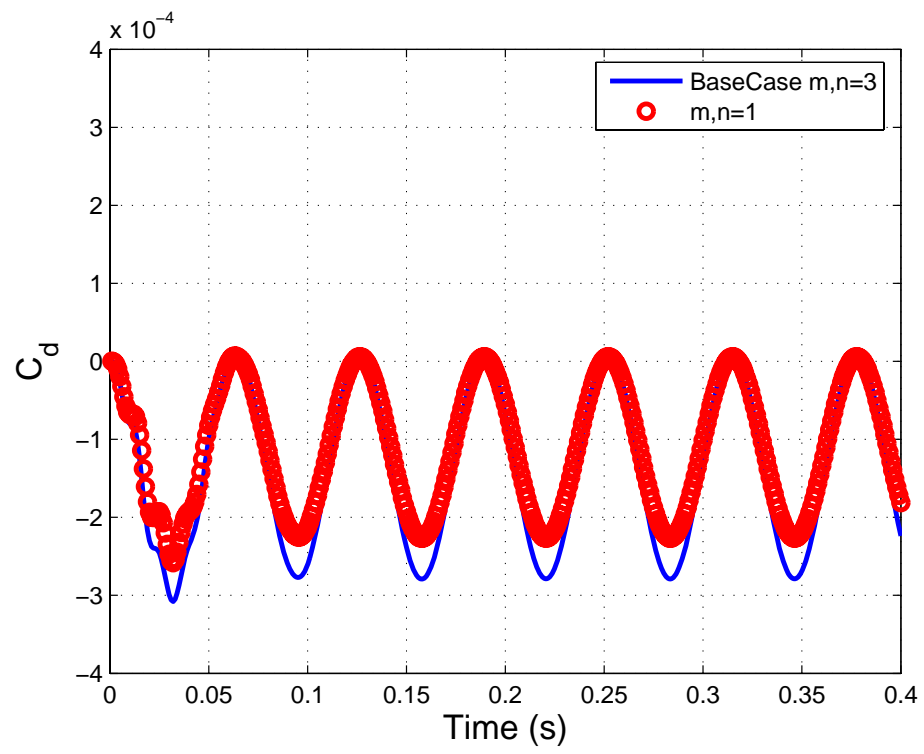

Figure 4.30: Plot of $C_{d}$ Vs Time for different number of structural modes for a plunging wing 


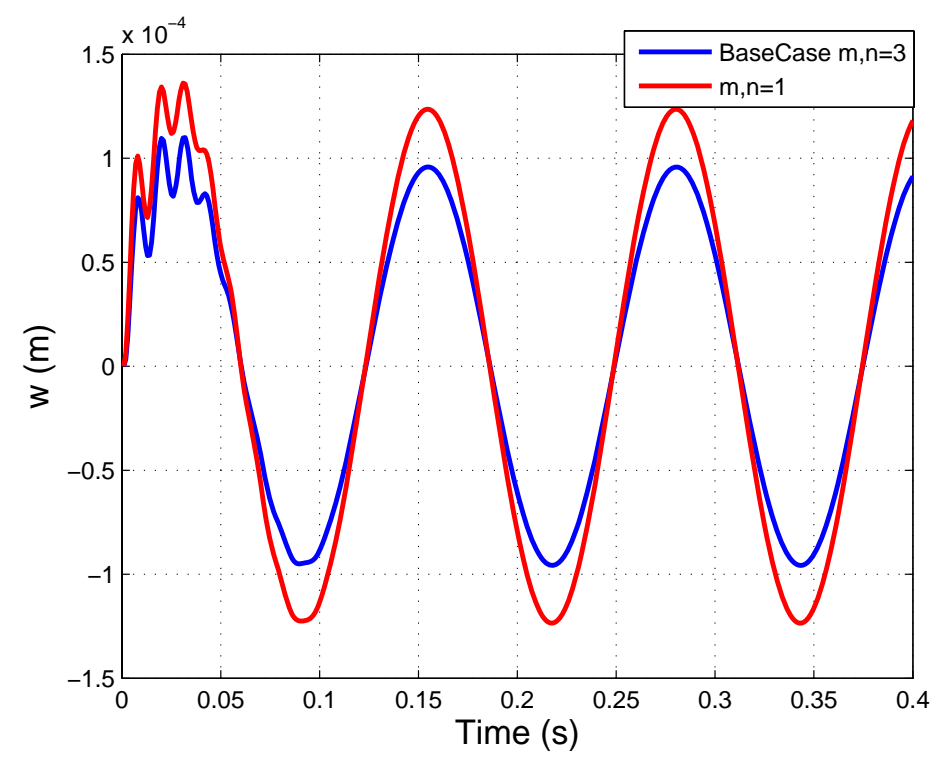

Figure 4.31: Plot of wing's center Vs Time for different number of structural modes for a plunging wing

modes. The lift coefficient data is found to be a match, as seen in Figure 4.29. The drag coefficient of the base case has troughs with much higher magnitudes Figure 4.30. The wing's deformation is better approximated with multiple modes. The pressure on the membrane is influenced by the dynamic motion of the wing, and the deformations of the membrane. Since the membrane deformations are small as compared to the prescribed plunging motion, it has a minor contribution to the pressure. Now, for the case of plunge, the wing is almost flat as the deformations are minor, as seen in Figure 4.31 for the wing's center. The aeroelastic influence on the load calculation is therefore minor too. Due to this reason, the $C_{l}$ calculated by the base case and the case with one structural mode are the same. In a linear sense, 
as the approximated deformation decreases, the load decreases by approximately the same factor. In the absence of angle of attack, the induced drag would be expected to be zero. Although, the thrust calculated for the minor deformations is minor, the drag coefficient calculated by the $m=n=1$ case is lower than the base case. This is attributed to the deformation calculated by the base case. When multiple modes are used in the approximation of deformations, the peak of the deformation is always forward of the midchord due to the pressure distribution, as seen in the previous section for constant angle of attack. This leads to higher slopes of the deformations in the forward part of the wing. Due to this, the pressure's streamwise component, which is the thrust, yields a higher value. On the other hand, the pressure's streamwise components calculated aft of the deformation's peak on the wing have lower values in the negative direction, which is the drag. Thus, more thrust is produced from the forward section, and less drag is produced from the backward section. The sum produces a higher net thrust on the wing. As seen in Figure 4.30, the values of the drag coefficient are negative. The negative drag values are thrust and thus contribute in the forward motion of the wing. Also, the wing's center point seems to follow the same trend for both cases. The membrane keeps on oscillating as cyclic loads act on it. It is also apparent from Figure 4.31 that the membrane deformation reaches a periodic steady state after 2 periods of oscillation. 


\subsubsection{Variation in plunge amplitude}

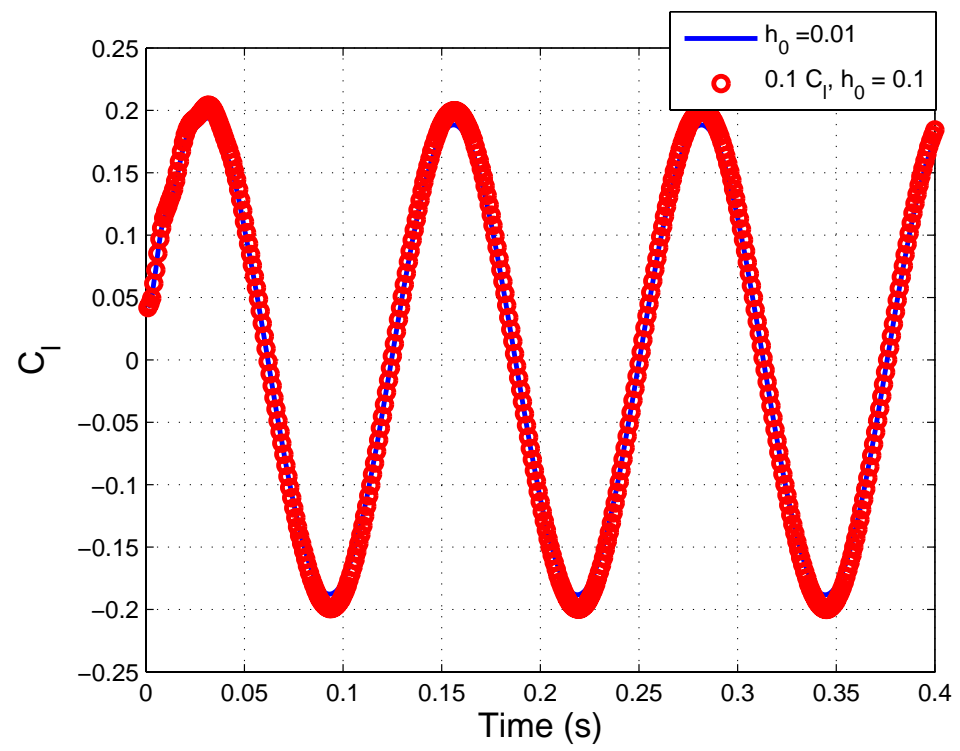

Figure 4.32: Plot of $C_{l}$ Vs Time for different plunge amplitudes for a plunging wing

The base case with plunge amplitude $h_{0}=0.01 \mathrm{~m}$ is compared with a case having a higher plunge amplitude of $h_{0}=0.1 \mathrm{~m}$. In a linear sense, it would be expected that the loads would be 10 times higher than the base case. For this reason, the value of $C_{l}$ for the $h_{0}=0.1 \mathrm{~m}$ case is multiplied by 0.1 when plotting it as a function of time, which is shown in Figure 4.32. This is done to make the $C_{l}$ plots of the same order, which gives more clarity in visual comparison. The lift even after correcting for the higher amplitude of motion is slightly higher for the $h_{0}=0.1 \mathrm{~m}$ case. This can be attributed to the nonlinear effect of large motion and the fact that the shed vortices will be spread out more vertically for higher plunge amplitude $h_{0}$. Otherwise, the 


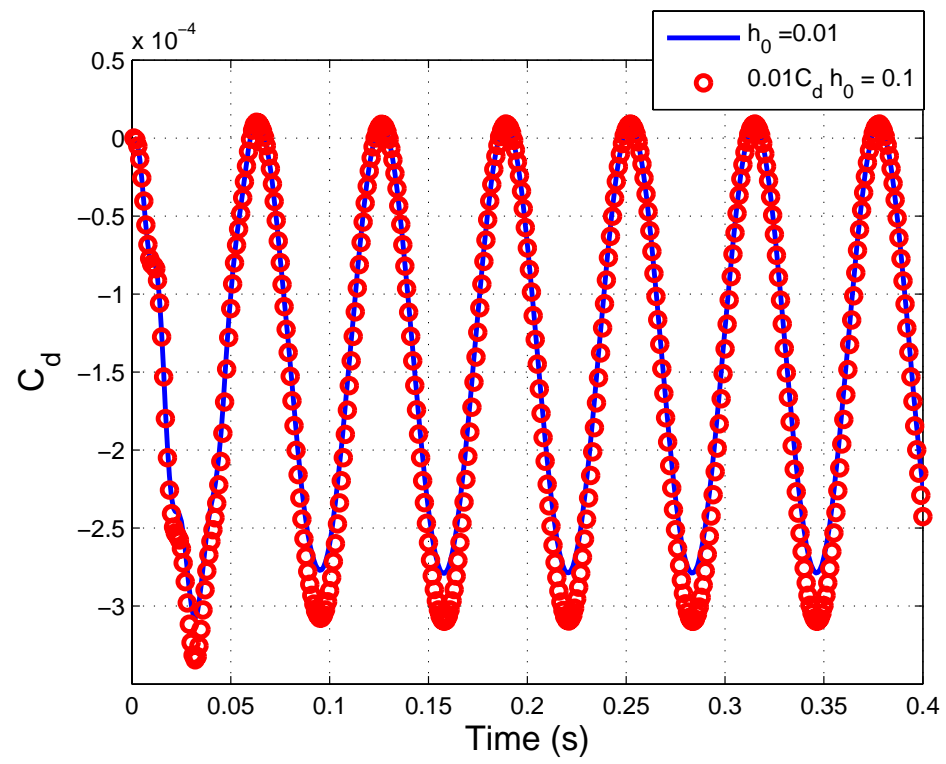

Figure 4.33: Plot of $C_{d}$ Vs Time for different plunge amplitudes for a plunging wing

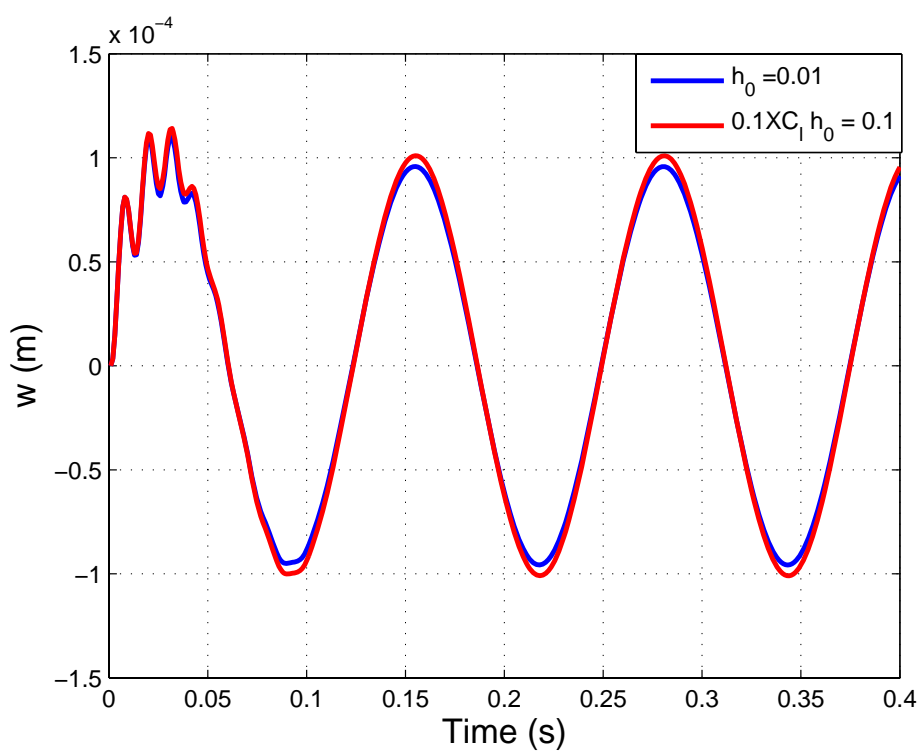

Figure 4.34: Plot of Wing's center Vs Time for different plunge amplitudes for a plunging wing 
lift coefficients match well with each other, concluding that for the 2 plunge cases considered, the $C_{l}$ is predominantly proportional to the plunge amplitude.

Figure 4.34 shows the deformation of the membrane for the two cases. As the $h_{0}=0.1 \mathrm{~m}$ case has around 10 times as much aerodynamic load as the $h_{0}=0.01 \mathrm{~m}$ case, it is expected that the deformation will be 10 times more for the $h_{0}=0.1 \mathrm{~m}$ case. To take this into account, the deformation for the $h_{0}=0.1 \mathrm{~m}$ case is multiplied by 0.1 . Even after this correction, the deformation at $h_{0}=0.1 \mathrm{~m}$ case is slightly higher because the corrected loads at $h_{0}=0.1 \mathrm{~m}$ are slightly higher.

Figure 4.33 shows the drag coefficient as a function of time for the two cases. Since the drag/thrust comes from the component of the pressure in the direction of the flow, it is a nonlinear (purely quadratic) expression. The increase in the pressure by a factor of 10 and the increase in slopes by a factor of 10 leads to an increase in the drag coefficient by a factor of 100 . Thus, to compare the two cases, the drag coefficient for $h_{0}=0.1 \mathrm{~m}$ case is multiplied by 0.01 . And as expected, the corrected drag coefficients match, with the drag coefficient being slightly larger for the $h_{0}=0.1$ $\mathrm{m}$ case (as the corrected loads and corrected deformation are larger for $h_{0}=0.1 \mathrm{~m}$ case). Also, as expected the cases lead to thrust because the thrust generated near the leading edge due to the high pressure and deformation slope is much higher than the drag generated from to the lower pressure and deformation slope at the trailing 
edge. It should be noted that the present analysis does not take into account the leading edge suction which can have a significant impact on the total thrust.

\subsubsection{Variation in reduced frequency}

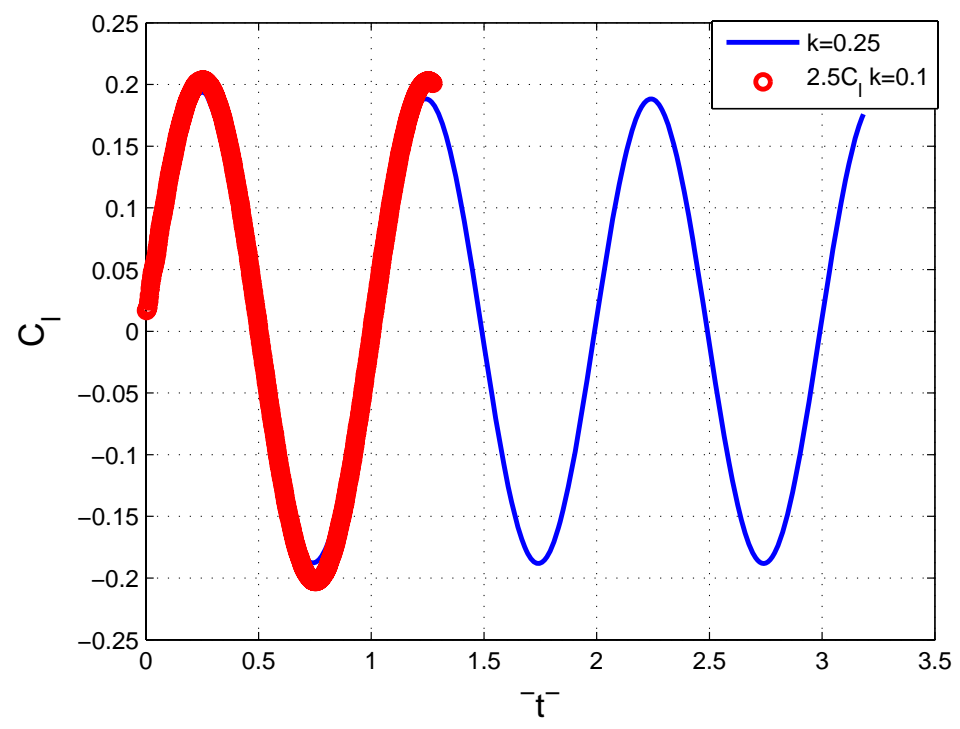

Figure 4.35: Plot of $C_{l}$ Vs Time for different reduced frequencies for a plunging wing

The base case with reduced frequency of $k=0.25$ is compared with a frequency of $k=0.1$. In a linear sense again, as the frequency is decreased from 0.25 to 0.1 , the pressure would be expected to decrease by a factor of 2.5 since we are considering a case of plunge and the magnitude of the effective angle of attack for the plunge case is derived from $\dot{h}$ and thus proportional to $h_{0} \times k$. For clarity in comparison, the $C_{l}$ for the $k=0.1$ case is multiplied by 2.5 and plotted in Figure 4.35 . Also, since 


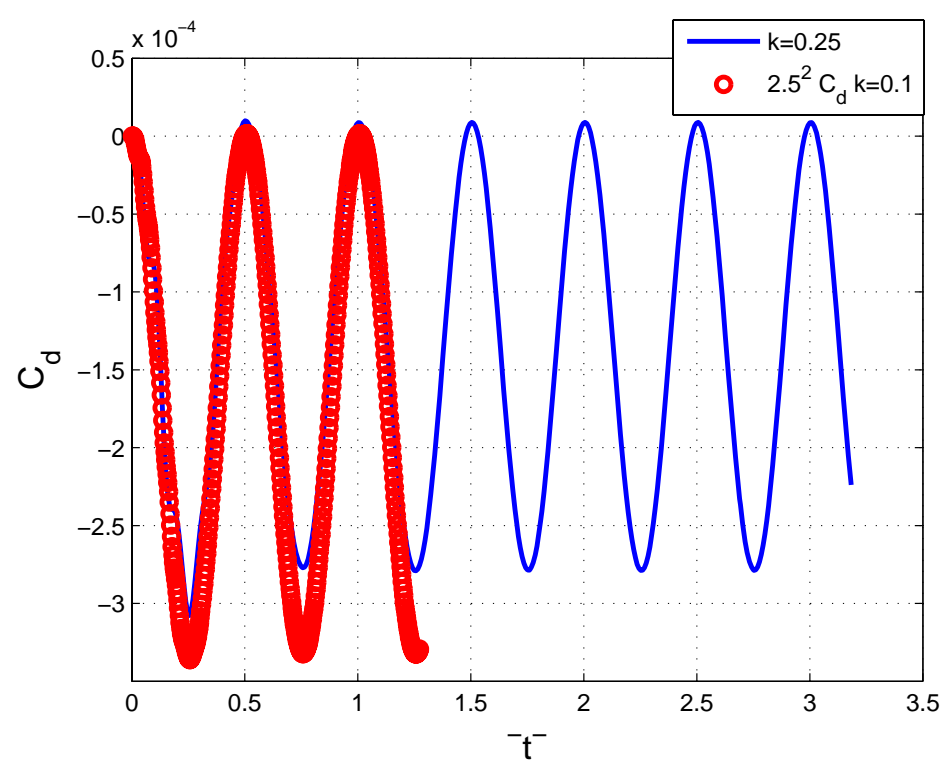

Figure 4.36: Plot of $C_{d}$ Vs Time for different reduced frequencies for a plunging wing

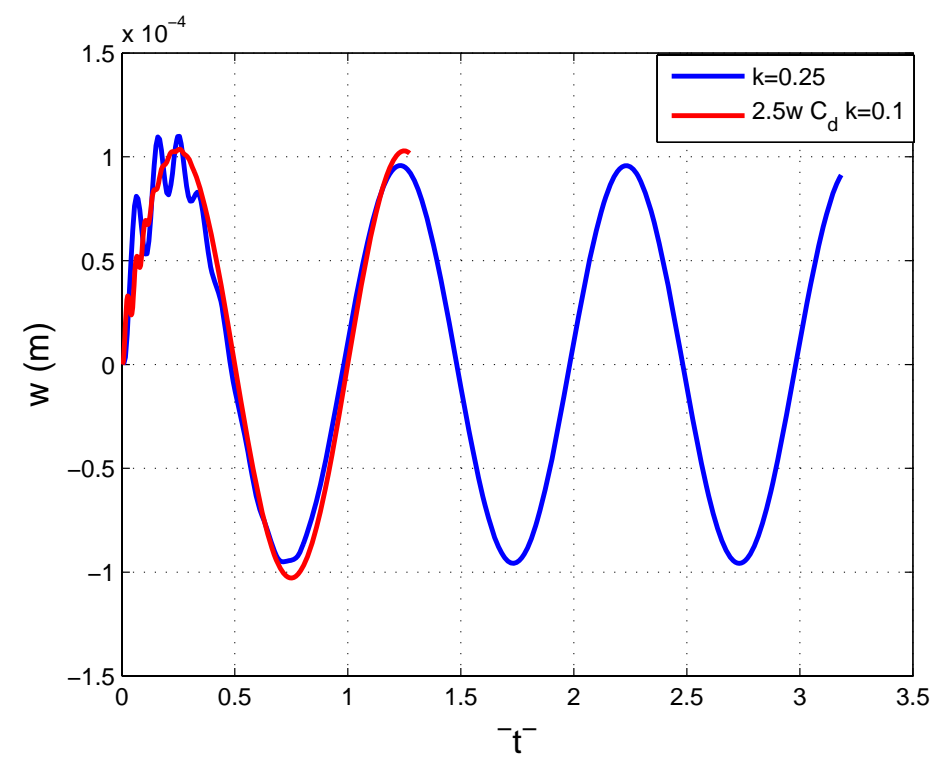

Figure 4.37: Plot of Wing's center Vs Time for different reduced frequencies for a plunging wing 
the frequency is changed, the time on the $x$ scale is changed to a non dimensional temporal parameter given by,

$$
\bar{t}=\frac{t}{T}
$$

where, $\mathrm{T}$ is the period

The change in the scale for the $x$ axis squeezes the data for the $k=0.1$ case as it undergoes fewer oscillations, but the plotted data is much easier to analyze. The correction of the results is the same as the correction imposed for a change in the plunge amplitude. The lift coefficient and deformation change by a factor of 2.5 , and the drag coefficient changes by a factor of $2.5^{2}$. There is slight difference in the magnitude and phase of the results. The lower reduced frequency has higher loads and lower phase lag relative to the plunge motion as expected from Theodorsen's function.

\subsubsection{Variation in stiffness}

In this study, the base case with a pre-stress stiffness of $S_{\text {stress }}=100 \mathrm{~N} / \mathrm{m}$ is compared with a case where the pre-stress is $S_{\text {stress }}=10 \mathrm{~N} / \mathrm{m}$. In the previous two comparisons, the varied parameter changed the input prescribed motion, which changed the aerodynamic loads and thus the structural deformation. In this case, however, 


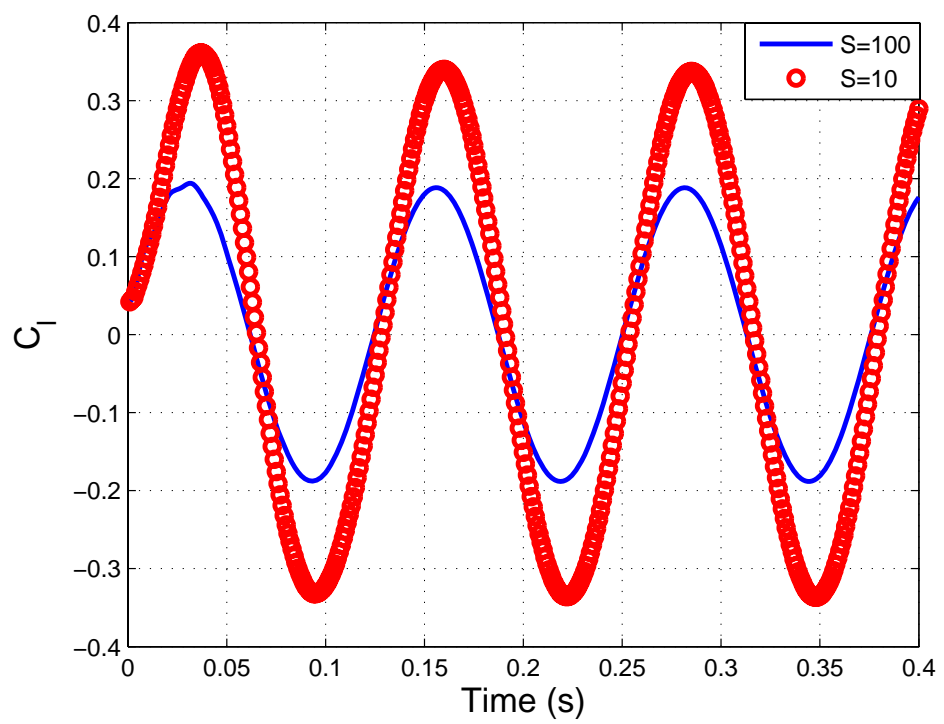

Figure 4.38: Plot of $C_{l}$ Vs Time for different pre-stresses for a plunging wing

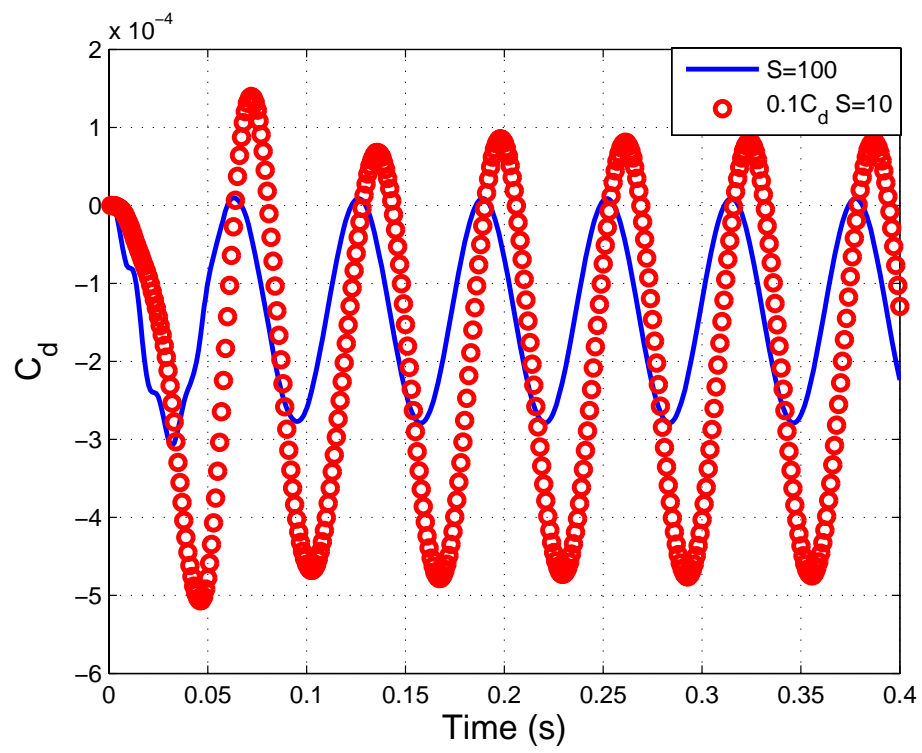

Figure 4.39: Plot of $C_{d}$ Vs Time for different pre-stresses for a plunging wing 


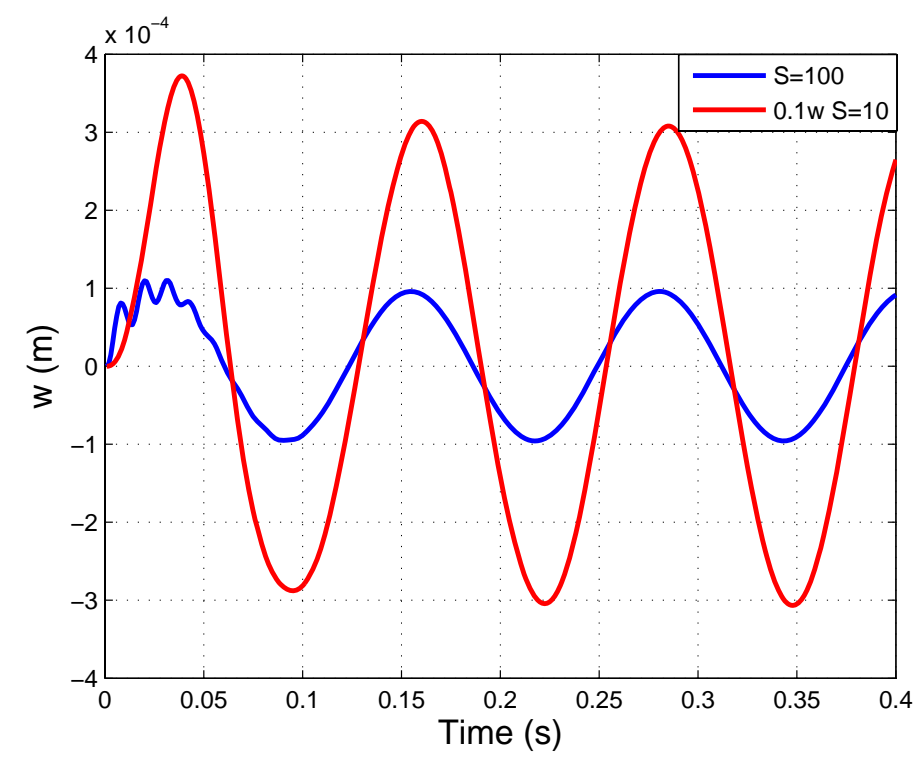

Figure 4.40: Plot of Wing's center Vs Time for different pre-stresses for a plunging wing

the varied parameter has a direct influence on the structural deformation. As the pre-stress stiffness is decreased, higher membrane deformation is expected. This is evident in Figure 4.40, which shows that the deformation is more than 10 times that of the base case. The lift rises for lower stiffness, as seen in Figure 4.38. This is because, as the membrane deformation rises, the pressure on the wing rises. However, considerable change is observed in the drag coefficient results, as seen in Figure 4.39. This is because, as the stiffness is reduced and the deformation rises, the pressure acting on the membrane gets more inclined toward the freestream direction resulting in a much higher component of the pressure as compared to the previous studies, resulting in higher thrust. The thrust generated is more than 10 times than the 
thrust generated by the base case.

\subsection{Study of loads for a pitching and plunging wing at different phases}

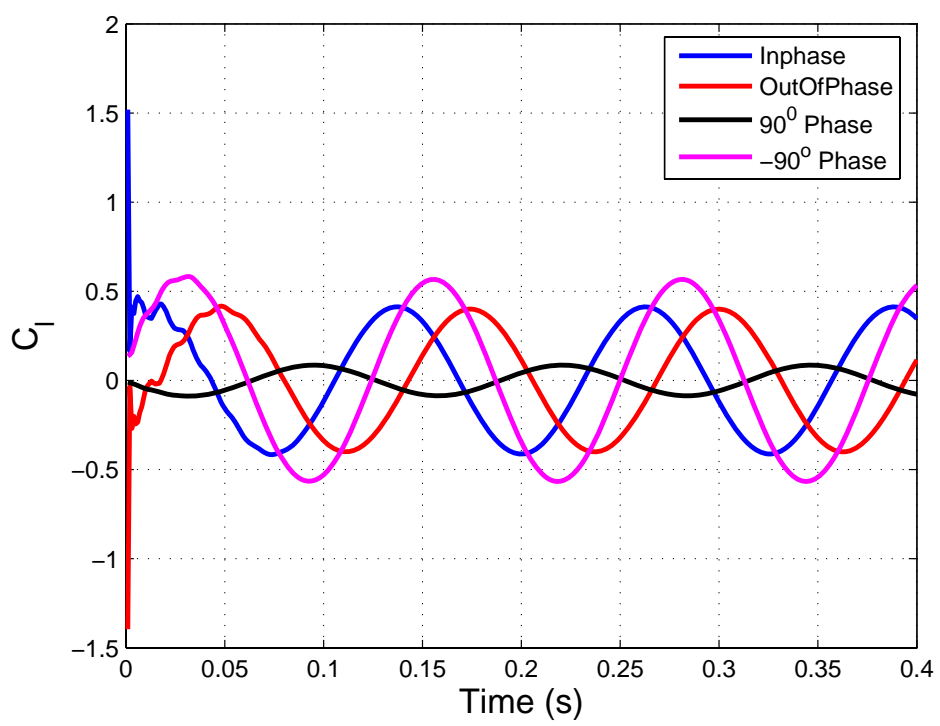

Figure 4.41: Comparison of $C_{l}$ for a pitching and plunging wing at different phases

The rectangular wing considered in this case has a chord length and span of $0.1 \mathrm{~m}$ and $0.5 \mathrm{~m}$ respectively. The wing oscillates at an angle of attack amplitude of $\alpha_{0}=5^{\circ}$, and plunges at a plunge amplitude of $h_{0}=\pi / 180 \mathrm{~m}$. It has a reduced frequency of 0.25. The density of air is $\rho=1 \mathrm{~kg} / \mathrm{m}^{3}$, and $m=n=3$ number of structural modes are used to estimate the deformation. The stiffness of the membrane is $S_{\text {stress }}=100$ 


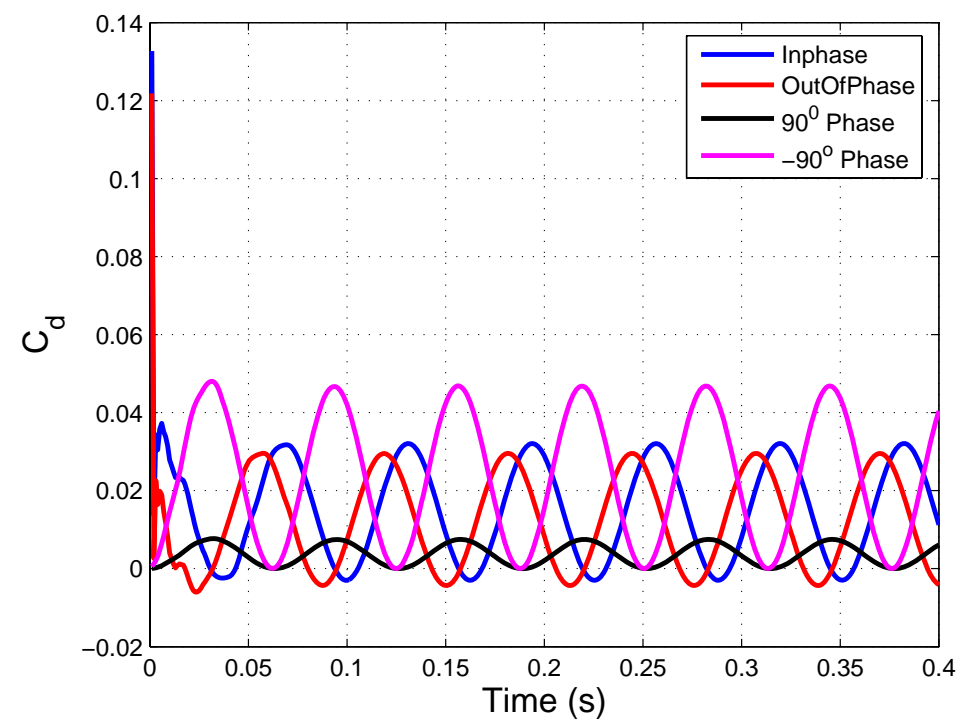

Figure 4.42: Comparison of $C_{d}$ for a pitching and plunging wing at different phases

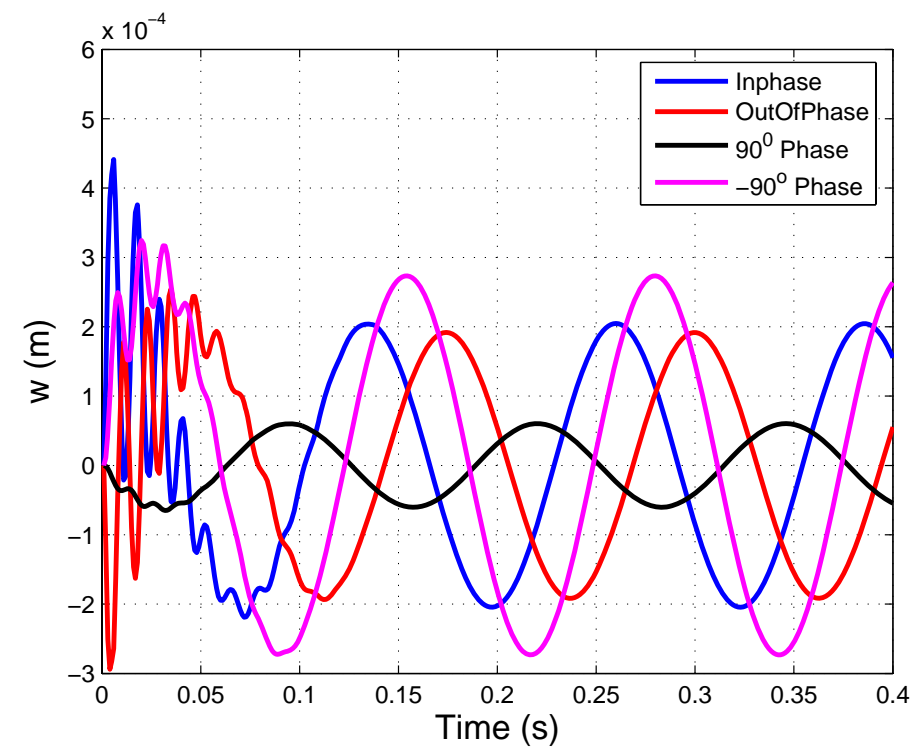

Figure 4.43: Comparison of $w$ for a pitching and plunging wing at different phases 
$\mathrm{N} / \mathrm{m}$. The value of the plunge amplitude has been selected such that,

$$
\frac{\dot{h}}{V_{\infty} \alpha} \approx 1
$$

where the freestream velocity $V_{\infty}=10 \mathrm{~m} / \mathrm{s}$.

The rigid wing structural velocity's and the freestream velocity's component normal to the wing is given by,

$$
\begin{aligned}
V_{\text {struc }} & =\left(V_{\text {rigid }}+V_{\infty}\right) \cdot \vec{n} \\
V_{\text {struc }} & =-h_{0} w_{0} \sin w_{0} t+R_{c} \alpha_{0} w_{0} \sin \left(w_{0} t+\phi\right)-V_{\infty} \alpha_{0} \cos \left(\omega_{0} t+\phi\right) \\
V_{\text {struc }} & =-h_{0} w_{0} \sin w_{0} t-V_{\infty} \alpha_{0} \sin \left(90-\left(\omega_{0} t+\phi\right)\right)+R_{c} \alpha_{0} w_{0} \sin \left(w_{0} t+\phi\right)(4.5) \\
& =-h_{0} w_{0} \sin w_{0} t+V_{\infty} \alpha_{0} \sin \left(\left(\omega_{0} t+\phi\right)-90\right)+R_{c} \alpha_{0} w_{0} \sin \left(w_{0} t+\phi\right)(4 .
\end{aligned}
$$

where, $R_{c}$ is the distance from the control point to the pivot point.

The velocity due to the freestream velocity and the plunge are the major components of $V_{\text {struc }}$, whereas the pitch component is the minor term. From Eq. 4.6, it can be seen that when the plunge and the pitch are in-phase, i.e $\phi=0$, there exists a phase of $-90^{\circ}$ in between the velocity component due to the freestream and the one due to plunge. Similarly when the two velocities are out-of-phase, there exists a phase of $90^{\circ}$ in between them. During both these cases the maximum and the minimum 
velocity remain the same. Thus, the amplitude of the lift coefficient for the in-phase and out-of-phase are the same. When the phase is $\phi=90^{\circ}$, the velocity amplitudes subtract yielding a lower total $V_{\text {struc }}$, which results in a lower $C_{l}$. And when the phase is $\phi=-90^{\circ}$, the amplitudes of the velocities add to give a higher $C_{l}$. Figure 4.41 shows this trend in the $C_{l}$ results.

Similar results can be seen in the drag coefficients in Figure 4.43. The difference in the amplitudes of the drag coefficients for the in-phase and out-of-phase cases exist because of the deformation, which causes a relative change in angle of attack. The drag results do not take the leading edge drag into account.

The deformation on the surface of the wing for the in-phase and out-of-phase cases go through a transition period before they oscillate in a periodic steady state. The transition is due to the different directions in which the plunge and pitch deflect the membrane. The $\pm 90^{\circ}$ phases do not transition as the pitch and the plunge cause deformation in the same direction. 


\section{Chapter 5}

\section{Conclusion}

With the goal of developing a tool that could be used in the preliminary aeroelastic analysis of MAVs, the aeroelastic model is developed. The unsteady aerodynamics of the model is based on the vortex lattice method. The unsteady aerodynamic code has been validated with a 2-dimensional VLM, lifting line theory,and the 3-dimensional aerodynamics has been validated with the doublet lattice method. To ascertain that the wake effects of the flow are captured, the wake locations of the 3-dimensional code were matched with those of the 2-dimensional VLM. Grid discretization study was performed to ensure convergence with different number of panels on the wing. The aerodynamic program is then interfaced with the structural code. The structural code is designed for a rectangular wing modeled by a rigid boundary structure sup- 
porting a membrane with the properties of nylon. The deformation of the structure is calculated using Fourier series. The coupled model is studied for constant angle of attack, oscillating plunge and pitch. For the studies conducted, various parameters such as the pitch amplitude $h_{0}$, structural stiffness $S_{\text {stress }}$, reduced frequency $(\mathrm{k})$, and the number of structural modes $(m, n)$ are varied. The conclusions drawn from the analysis of the results are outlined below:

- The unsteady VLM developed for the project is valid for pitching, plunging, and pitch and plunge dynamics.

- For constant angle of attack, the deformation calculated by multiple structural modes are more accurate. For the case considered, $m=n=3$ structural modes are sufficient to estimate the loads and deformations.

- For constant angle of attack, as the stiffness of the membrane is increased, lower deformation is experienced, which in turn yields a lower lift and lower drag.

- For an oscillating plunge motion, the use of multiple mode shapes in the approximation of the deformation yields more accurate and physically correct results.

- For an oscillating plunge motion, as the plunge amplitude is increased, it is 
observed that the lift and the deformation on the wing increase by the factor by which the amplitude is increased. However, the thrust increases/decreases by the factor squared.

- For an oscillating plunge motion, as the reduced frequency is increased/decreased, it is observed that the lift and the deformation on the wing increases/decreases by the factor by which the reduced frequency is increased/decreases and again, the thrust increases/decreases by the factor squared.

- For an oscillating plunge motion, as the stiffness of the membrane is reduced, higher deformation is experienced, which in turn yields a higher lift and much higher thrust.

- For an oscillating plunge motion, in all the cases considered, thrust is generated. This thrust is due to the pressure on the wing and does not include the leading edge suction. Thus, the thrust is produced due to the deformation of the wing and will not be present for a rigid wing oscillating in plunge.

- For an oscillating pitch and plunge motion, the $C_{l}$ increases for phase lag of $-90^{\circ}$, decreases for $-90^{\circ}$, and the $C_{l}$ amplitude stays the same for in-phase and out-of-phase motion 


\section{Bibliography}

[1] B. Singh and I. Chopra. Dynamics of insect-based flapping wings: Loads validation. In Proceedings of the 47 th AIAA/ASME/ASCE/ASC Structures, Structural Dynamics, and Materials Conference, Newport, Rhode Island, May 2006.

[2] P. Barnswell F. Boria D. Claxton J. Clifton J. Cocquyt K. H. Lee S. Mitryk M. Abdulrahim, R. Albertani and P. Ifju. Design of the university of florida surveilance and endurance micro air vehicles. Technical report, University of Florida, 2003.

[3] F. Boria D. Claxton J. Clifton J. Cocquyt A. Crespo C. Francis P. Ifju B. Johnson S. Jung K. H. Lee R. Albertani, P. Barnswell and M. Morton. University of florida biologically inspired micro air vehicles. Technical report, University of Florida, 2004.

[4] Micro Air Vehicle Laboratory. Development of a composite bendable-wing micro 
air vehicle. Technical report, University of Florida, 2006.

[5] B. Stanford and P. Ifju. Membrane micro air vehicles with adaptive aerodynamic twist: Numerical modeling. AIAA/ASME/ASCE/AHS/ASC Structures, Structural Dynamics, and Materials Conference, 48(1), April. 2007.

[6] M. Wolf R. von Busse Y. WInter A. Hedenstrom, L.C. Johansson and G.R. Spedding. Bat flight generates complex aerodynamic tracks. Technical report, 10.1126/Science.1142281, 2007.

[7] Z. Wang. Time-Domain Simulations of Aerodynamic Forces on ThreeDimensional Configurations, Unsteady Aeroelastic Responses, and Control by Neural Network Systems. PhD thesis, Virginia Polytechnic Institute and State University, Blacksburg, Virginia, May. 2004.

[8] C. E. Lan. The unsteady quasi-vortex-lattice method with applications to animal propulsion. Journal of Fluid Mechanics, 93(4):747 - 765, 1979.

[9] F. Nitzsche and D.G. Opoku. Acoustic validation of a new code using particle wake aerodynamics and geometrically-exact beam structural dynamics. The Aeronautical Journal, 45(2880):257-267, June. 2005.

[10] D. G. Opoku. Aeroelastic and aeroacoustic modelling of rotorcraft. Master's thesis, Carleton University, Ontario, Canada, 2005. 
[11] D.J. Lee and S. U. NA. Numerical simulations of wake structures generated by rotating blades using a time marching, free vortex blob methods. European Journal of Mech - B/Fluids, 18(1):147 - 159, 1999.

[12] B.J. Maclean and R. A. Decker. Lift analysis of a variable camber airfoil using the discrete vortex blob method. AIAA Journal, 32(7):1525 - 1527, July 1994.

[13] P. Anusonti-Inthra. Development of rotorcraft wake capturing methodology using fully coupled cfd and particle vortex transport method. In Presented at the American Helicopter Society 62nd Annual Forum, Hampton, VA, May 2006. AHS-2006.

[14] J. Katz and A. Plotkin. Low-Speed Aerodynamics. Cambridge University Press, San Diego, California, US, 2001.

[15] M. J. Bhagwat L. G. Leishman and A. Bagai. Free-vortex filament methods for the analysis of helicopter rotor wakes. Journal of Aircraft, 39(5):759 - 775, Sept-Oct 2002.

[16] D. A. Wachspress J. F. Horn, D. O. Bridges and S. L. Rani. Implementation of a free vortex wake model in real time simulation of rotorcraft. In Presented at the American Helicopter Society 61st Annual Forum, University Park, PA And Ewing, NJ, June 2005. 
[17] M. J. Patil. From fluttering wings to flapping flight: The energy connection. In Proceedings of the 42nd Structures, Structural Dynamics and Materials Conference, Seattle, Washington, April 2001. AIAA-2001-1460, (To appear in Journal of Aircraft).

[18] A. Natarajan. Aeroelasticity of Morphing Wings Using Neural Networks. PhD thesis, Virginia Polytechnic Institute and State University, Blacksburg, Virginia, July. 2002.

[19] K. Karamcheti. Principles of Ideal Fluid Aerodynamics. Malabar, Florida. Krieger Publishing Company, 1980.

[20] S. Preidikman. Numerical Simulations of Interactions Among Aerodynamics, Structural Dynamics, and Control Systems. PhD thesis, Virginia Polytechnic Institute and State University, Blacksburg, Virginia, October. 1998.

[21] R. L. Bisplinghoff, H. Ashley, and R. L. Halfman. Aeroelasticity. Addison-Wesley Publishing Co., Reading, Massachusetts, 1955.

[22] A. Ugural and S. Fenster. Advanced Strength And Applied Elasticity. Englewood Cliffs, NJ. P T R Prentice Hall, 1995.

[23] S. Banerjee. Low speed unsteady aerodynamics. Technical report, Virginia Tech, 2005. 
[24] J. D. Anderson. Fundamentals of Aerodynamics. Maryland. McGraw Hill, 2001.

[25] E. Albano and W. P. Rodden. A doublet-lattice method for calculating lift distributions on oscillating surfaces in subsonic flow. AIAA Journal, 7(2):279285, Feb. 1969. 